Introduçãö de Fontes e de Sumidouros em escoamentos incompressíveis bidimensionais por intermédio do Método da Fronteira Imersa

Santos Alberto Enriquez Remigio

\author{
DISSERTAÇÃO APRESENTADA \\ $\mathrm{AO}$ \\ INSTITUTO DE MATEMÁTICA E ESTATÍSTICA \\ DA \\ UNIVERSIDADE DE SÃO PAULO \\ PARA OBTENÇÃO DO GRAU \\ DE \\ MESTRE EM MATEMÁTICA APLICADA
}

Área de Concentração:

ANÁLISE NUMÉRICA

Orientador: Prof. Dr. Alexandre Megiorin Roma

Durante a elaboração deste trabalho, o autor recebeu apoio financeiro do CNPq.

-São Paulo, Janeiro de 2000- 


\section{Introdução de Fontes e de Sumidouros em escoamentos incompressíveis bidimensionais por intermédio do Método da Fronteira Imersa}

\footnotetext{
Este exemplar corresponde à redação final devidamente corrigida e defendida por Santos Alberto Enriquez Remigio e aprovada pela comissão julgadora.
}

São Paulo, 10 de Janeiro de 2000.

Banca examinadora

Prof. Dr. Alexandre Megiorin Roma

(IME-USP)

Prof. Dr. Luis Carlos de Castro Santos (IME-USP)

Prof. Dr. Aristeu Silveira Neto

(UFU) 
....A MIS PADRES. 


\section{Agradecimentos}

A Deus, por cuidar de meus pais e família, por guiar nossos caminhos e nos permitir conhecer grandes amigos.

Aos meus pais, por tudo o que fizeram e pelo amor que me dão. Foram eles que me ensinaram a ter forças para continuar avançando, apesar disto significar estarmos distantes.

Ao meu irmão Hilario e à minha irmã Juana, por serem meus melhores amigos, dispostos a me ensinar, a me escutar e por manterem sempre forte o laço familiar. Ao meu cunhado Emilio, por ser como um irmão, disposto a apoiar a mim e à minha família. À minha cunhada Elena por seus conselhos e apoio.

Aos meus sobrinhos: Arturito, Yesenia, Cristian, Melody, Rocio, Katy e Frida por serem meus amiguinhos e por toda a alegria que me dão.

À minha tia Cristina por ser como uma mãe para mim. À minha prima e amiga Isabelita por tudo o que fez por nós. E a todos meus familiares por estarem sempre conosco nas horas boas e também nas horas difícies.

Aos meus professores: Felix Escalante, Roxana Lopez, Mario Santiago, Rolf Schröeder, Alejandro Hidalgo, Gimaray por suas valiosos ensinamentos e orientações.

Ao meu orientador, Professor Alexandre M. Roma, por o grande apoio, confiança, ensinamento e paciência. Sempre disposto a me escutar e dar sua sábia opinião. Muito obrigado Professor Roma!

Ao Professor Luis Carlos de Castro Santos, por suas valiosas idéias e críticas durante o desenvolvimento de minha pesquisa e na defesa de minha dissertação.

Ao Professor Aristeu Silveira Neto, por estar presente na banca de minha defesa de dissertação, por suas sugestões, críticas e apoio na continuação de minha pesquisa.

Ao Masaishi, por ter emprestado o apoio de seu programa o que me permitiu avançar minha pesquisa.

Ao pessoal da Divisão de Bioengenharia do InCor: Dra. Idágene, Dr. Sérgio Furuie, Dr. Marco Gutierrez, Dra. Ana Cristina, pelas discussões e sugestões na desenvolvimento de minha pesquisa.

Aos Professores: Helena M. A. de Castro, Oswaldo Rio Branco de Oliveira, Oscar Vilcachagua, pelas ensinamentos e apoio em todo momento. 
À Vera por sua linda forma de ser, capaz de dar tudo por nós, por todos os momentos, inesquecíveis, que me dá dia a dia. Não pensei que os sonhos fossem possíveis até que a conheci.

Aos meus amigos que fizeram possíveis que a minha estadia no Brasil fosse muito boa: Hernan, Ricardo Gutierrez, Antonio, Nelson, Calderon, Xyoby, Julio, Elmer, Magen, Beto, Sonia, Betty, Katia, Esteban, Delhi, Lobo, Jocerei, Cibele, Cardenas, Juan, Raul Jimenez, Karina, Jackeline, Bernardo, Alice, Lucy, Ricardo Antunis da Rocha, Said, Olga, Claudia, Rudimar, Liliane, Glaucio, Calixto, Walter, Uirá, Leandro, Emmanuel, Franklin, Maria do Carmo.

À todos os professores do IME-USP e funcionários dos laboratórios, secretarias, biblioteca, seção da pós, funcionários da sala de café que de forma direta e/ou indireta contribuiram para a realização deste trabalho. 


\section{Resumo}

Uma ferramenta para bioengenheria com alto impacto social é a simulação computacional do funcionamento do Dispositivo de Assistência Ventricular (DAV). Os DAV's são empregados para manter a pressão e o fluxo de sangue, auxiliando a circulação sangüínea durante o ciclo cardíaco. Isto explica porque, de alguns anos para cá, o uso desses dispositivos vem crescendo como uma opção terapêutica para pacientes que esperam por um transplante cardíaco. A unidade de bombeamento do DAV é conectada ao coração do paciente por meio de tubos flexíveis. Surgem questões relevantes de bioengenheria relacionadas à mecânica de fluidos deste problema, especialmente em termos da forma dos tubos: Quais formas seriam as mais adequadas para a secção transversal? É a forma circular a melhor? As ondulações representam alguma vantagem? Nesse caso, onde elas deveriam estar localizadas?

Tendo em vista esta motivação científica, o objetivo deste trabalho é, seguindo os trabalhos de Peskin, apresentar, implementar e testar adequadamente fontes e sumidouros, elementos necessários na modelagem matemática da entrada e saída de fluido em tubos flexíveis.

O Método da Fronteira Imersa de Peskin é particularmente adequado para muitas aplicações na dinâmica de biofluidos envolvendo a interação entre fluidos viscosos incompressíveis e interfaces elásticas. Aqui, por simplicidade e não por uma restrição imposta pela metodologia, as simulações mostradas são realizadas apenas para modelos de problemas bidimensionais. 


\begin{abstract}
A tool to bioengineering with a high social impact is the numerical simulation of Ventricular Assist Devices (VAD's). VAD's are employed to maintain proper blood pressure and flux, assisting blood circulation during the cardiac cycle. In recent years, the use of these devices is increasing as a therapeutic option for patients waiting for a cardiac transplantation. The VAD pumping unit is connected to the patient's heart by flexible tubes. Relevant bioengineering questions arise related to the biofluid mechanics of this problem, especially in terms of the shapes of the tubes: "What are their proper cross sections? Is the circular one the best? Are tapers of any advantage? If so, where should they be placed?" Bearing this scientific motivation in mind, the goals of this work are, following Peskin's works, to present, to implement and to test properly sources and sinks, the modelling elements needed to simulate inflow and outflow conditions for an incompressible flow in flexible tubes. Peskin's Immersed Boundary Method is particularly suitable for many applications in biofluid dynamics involving the interaction between transient, incompressible viscous fluids and elastic interfaces. Here, for the sake of simplicity and not for a restriction imposed by the methodology, the simulations shown are performed only for two-dimensional model problems.
\end{abstract}




\section{Índice}

Lista de Figuras $\quad$ iii

Lista de Tabelas $\quad$ v

1 Introdução 1

1.1 Revisão da Literatura . . . . . . . . . . . . . . . . . . . . . . 3

1.2 Apresentação deste trabalho . . . . . . . . . . . . . . . . . 6

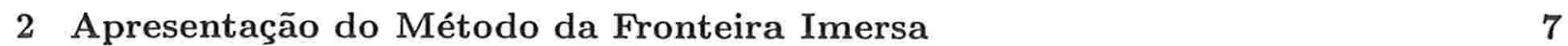

2.1 Modelo matemático . . . . . . . . . . . . . . . . . . . . 7

2.1.1 Resumo das equações do modelo matemático . . . . . . . . . . . . . 11

2.2 Método computacional . . . . . . . . . . . . . . . . . . . . . . 12

2.2.1 Notações e operadores de diferença empregados . . . . . . . . . . . . . . . 12

2.2.2 Discretização e resolução numérica do modelo matemático . . . . . . . . . 15

$2.3 \quad$ Algoritmo do Método da Fronteira Imersa . . . . . . . . . . . . . . . . 18

3 Modelagem de entrada e de saída de fluido no problema $\quad 20$

3.1 Escoamento entre placas paralelas e modelagem da vazão . . . . . . . . . . . . . 21

3.1.1 Escoamento entre placas paralelas . . . . . . . . . . . . . . . . . . 21

3.1 .2 Modelagem de vazão . . . . . . . . . . . . . . . . . . . . . 22

3.2 Escoamentos contendo uma fonte e um sumidouro . . . . . . . . . . . . . . . 24

3.2.1 Modelo adotado por Peskin para fonte e sumidouro . . . . . . . . . . . . 24

3.2.2 Inclusão de uma fonte e um sumidouro no método computacional . . . . . 26 
3.2.3 Algoritmo do Método da Fronteira Imersa contendo uma fonte e um sumidouro . . . . . . . . . . . . . . . . . . 28

3.3 Escoamentos contendo $k$ fontes e $k$ sumidouros $\ldots \ldots \ldots \ldots \ldots$

4 Testes numéricos $\quad 34$

4.1 Preliminares: normas e análise de convergência . . . . . . . . . . . . . . . 34

4.2 Resolução numérica das equações de Navier-Stokes $\ldots \ldots \ldots$. . . . . . . . . . 36

4.3 Introdução de uma fonte e um sumidouro no modelo . . . . . . . . . . . . . . 37

4.4 Escoamento laminar entre placas paralelas . . . . . . . . . . . . . . . . 46

4.4 .1 Modelo bidimensional . . . . . . . . . . . . . . . . . . . . 46

4.4 .2 Condições iniciais na simulação . . . . . . . . . . . . . . . . . 47

4.4 .3 Resultados . . . . . . . . . . . . . . . . . . . . . 48

5 Conclusão $\quad 54$

A Aproximação do Delta de Dirac $\quad 56$

A.1 Função impulso unitário . . . . . . . . . . . . . . . . . . 56

A.2 Núcleos de Dirac . . . . . . . . . . . . . . . . . . . 57

B Decomposição de um campo vetorial (caso periódico) $\quad 60$

B.1 Preliminares: definições e propriedades . . . . . . . . . . . . . . . . . . . 60

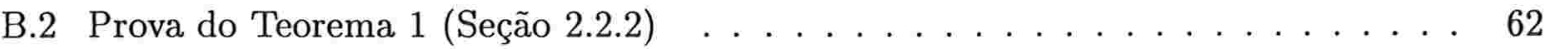

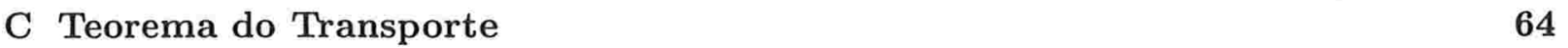

C.0.1 Derivada Material e teorema de transporte . . . . . . . . . . . . . 65

D Cálculo da área de polígonos $\quad 66$

D.1 Fórmula integral do cálculo da área . . . . . . . . . . . . . . . . 66 


\section{Lista de Figuras}

1.1 Dispositivo de Assistência Ventricular (DAV) . . . . . . . . . . . . . . 1

1.2 DAV intracorpóreo implantado em um paciente. . . . . . . . . . . . . 2

1.3 Unidade de bombeamento do DAV paracorpóreo desenvolvido no Instituto do Coração do Hospital das Clínicas da Faculdade de Medicina da Universidade de

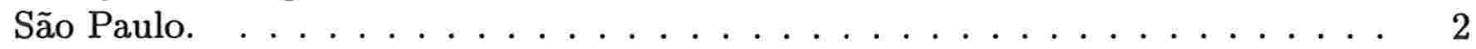

1.4 Uso do DAV paracorpóreo desenvolvido no InCor. . . . . . . . . . . . . . . . 3

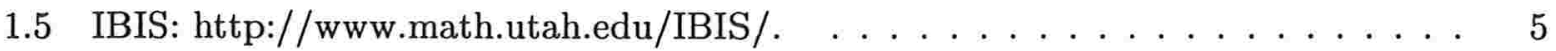

2.1 Forças atuantes na fibra no trecho $\mathbf{X}\left(s_{1}, t\right)$ e $\mathbf{X}\left(s_{2}, t\right) \ldots \ldots \ldots$

2.2 R é uma região arbitrária de domínio $\Omega . \ldots \ldots \ldots \ldots$

2.3 Malhas Euleriana ("o") e Lagrangiana ("॰"). . . . . . . . . . . . . . . . . . . 13

2.4 Gráfico da função discreta delta de Dirac bidimensional, $\delta_{h}\left(\mathrm{x}-\mathrm{x}_{0}\right)$, com centro no ponto $\mathrm{x}_{0}=(0.25,0.5)\left(h=\frac{1}{n}, n=64\right) \ldots \ldots \ldots \ldots \ldots \ldots \ldots$

2.5 Gráfico da função discreta delta de Dirac unidimensional, $d_{h}(x)\left(h=\frac{1}{n}, n=5\right) . \quad 15$

2.6 Uso da aproximação do delta de Dirac. . . . . . . . . . . . . . . . . . . . 17

3.1 (a) Escoamento laminar entre duas placas paralelas movido pelo gradiente de pressão; (b) perfil do escoamento em um plano paralelo ao plano yx. . . . . . . . 21

3.2 Superfície retangular paralela ao plano $y z \ldots \ldots \ldots \ldots \ldots$

3.3 Suprimento e remoção de fluido no domínio, $\Omega \ldots \ldots \ldots \ldots$. . . . . . . 26

3.4 Escoamentos com duas fontes e dois sumidouros e respectivas vazões. . . . . . . 29

3.5 Escoamentos com duas fontes e um sumidouro e respectivas vazões. . . . . . . . 33

4.1 Gráfico da $\Psi_{0}$ numa malha $64 \times 64 \ldots \ldots \ldots \ldots \ldots \ldots$

4.2 Localização inicial das circunferências: uma ao redor da fonte e outra ao redor do sumidouro. 
4.3 Medida da diferença entre a taxa de variação da área teórica e experimentalmente na simulação do escoamento de um fluido contendo uma fonte e um sumidouro;

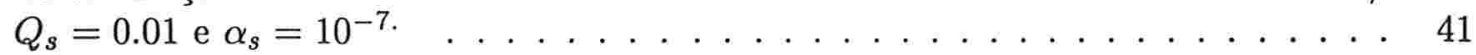

4.4 Razão entre as variações experimentais das áreas das circunferências ao redor da fonte e ao redor do sumidouro; $Q_{s}=0.01$ e $\alpha_{s}=10^{-7} \ldots \ldots \ldots \ldots$. . . . 42

4.5 Simulação do escoamento de um fluido contendo uma fonte e um sumidouro; malha $32 \times 32 ; Q_{s}=0.01$ e $\alpha_{s}=1 e-7, t=1.2 s . \ldots \ldots \ldots \ldots \ldots 4$

4.6 Simulação do escoamento de um fluido contendo uma fonte e um sumidouro; malha $64 \times 64 ; Q_{s}=0.01$ e $\alpha_{s}=1 e-7$ e $t=1.2 s . \ldots \ldots \ldots \ldots$

4.7 Modelo bidimensional para simular o escoamento entre placas paralelas. . . . . 46

4.8 Secçao transversal do modelo tridimensional adotado para o escoamento laminar dentro das placas; configuração inicial (tempo $=0.0$ ). Os quadrados de lado $L=4 h_{0}$, representam o domínio da fonte e do sumidouro com centros em $\mathbf{P}_{F}$ e

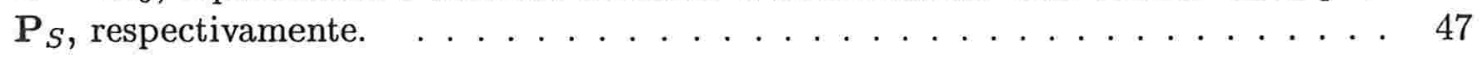

4.9 Valores da vazão $Q(t) . \ldots \ldots \ldots \ldots \ldots \ldots \ldots$

4.10 Perfil de velocidades na metade do canal. . . . . . . . . . . . . . 50

4.11 Quadro da simulação no tempo $\mathrm{t}=1.0$; malha $16 \times 16$ e $32 \times 32 . \ldots \ldots \ldots$

4.12 Quadro da simulação no tempo $t=1.0$; malha $64 \times 64 \ldots \ldots \ldots \ldots$

4.13 Quadro da simulação no tempo $\mathrm{t}=1.0$; malha $128 \times 128 \ldots \ldots \ldots \ldots$

A.1 Gráfico das funções $\phi(r):(1) \phi^{(1)} ;(2) \phi^{(2)} ;(3) \phi^{(1)} ;(4) \phi^{(4)} ;(5) \phi^{(5)} . \quad \ldots \ldots . \quad 59$

C.1 Região do espaço ocupado pelo fluido no instante $t, \Omega_{t}$. . . . . . . . . 64

D.1 Fronteira de $\Omega(\partial \Omega)$ com forma de polígono. . . . . . . . . . . . . . 67

D.2 Ángulos possíveis formados por $\mathbf{A}$ e $\mathbf{B}^{\perp}$ : (a) agudo e (b) obtuso. . . . . . . . 67 


\section{Lista de Tabelas}

4.1 Passo de projeção: erros cometidos, no campo de velocidade, e razão entre eles no tempo $t=0.3125 \mathrm{~s} . \ldots \ldots \ldots \ldots \ldots \ldots \ldots$

4.2 Escoamento laminar entre placas paralelas: erros cometidos, no campo de velocidade no meio do canal, e razão entre eles no tempo $t=1.0 \mathrm{~s} . \quad \ldots \ldots$. . . . . 49

4.3 Erros relativos no tempo $t=1.0 s . \ldots \ldots \ldots \ldots \ldots \ldots$ 


\section{Introdução}

Uma ferramenta para bioengenheria com alto impacto social é a simulação computacional do funcionamento do Dispositivo de Assistência Ventricular (DAV). Tipicamente, o DAV consiste de tubos flexíveis e uma bomba, as quais são empregadas para manter a pressão e o fluxo de sangue, auxiliando a circulação sangüínea durante o ciclo cardíaco. Alguns deles, os DAV paracorpóreos podem auxiliar um ou ambos os ventrículos por semanas ou meses. Outros, os intracorpóreos podem auxiliar o ventrículo esquerdo por anos! Isto explica porque, de alguns anos para cá, o uso desses dispositivos vem crescendo como uma opção terapêutica para pacientes que esperam por um transplante cardíaco [11]. Na Figura 1.1, mostra-se como é um DAV intracorpóreo. Dois tubos flexíveis são utilizados: um para conectá-lo ao ventrículo esquerdo e outro à aorta.

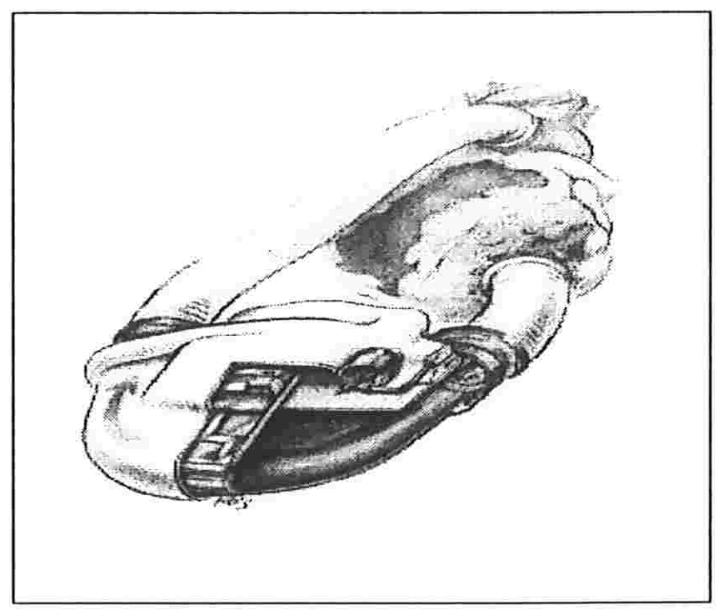

Figura 1.1: Dispositivo de Assistência Ventricular (DAV). 
A Figura 1.2 ilustra o DAV intracorpóreo implantado em um paciente.

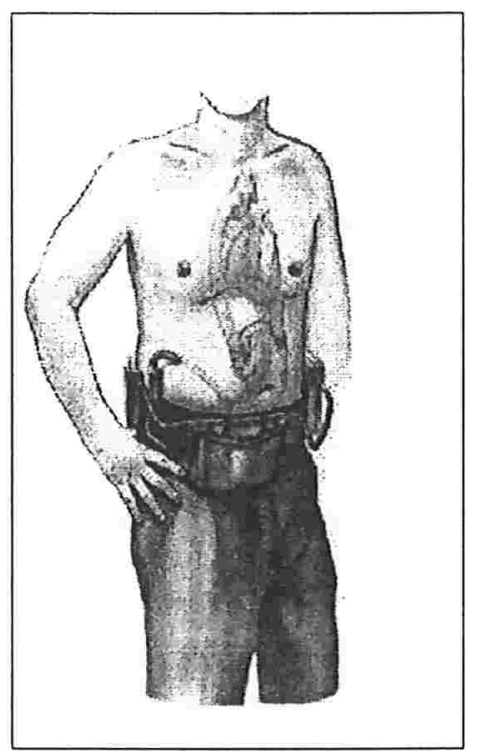

Figura 1.2: DAV intracorpóreo implantado em um paciente.

As figuras anteriores foram retiradas do sítio http://www.bgsm.edu/bgsm/surg-sci/ct/, o qual pertence ao Departamento de Cirurgia Cardiotoráxica do Baptist Medical Center da Universidade de Wake Forest.

No Instituto do Coração (InCor) do Hospital das Clínicas da Faculdade de Medicina da Universidade de São Paulo, foi desenvolvido um DAV paracorpóreo. Este consiste de uma unidade de bombeamento (Figura 1.3), e uma unidade de controle (não mostrada).

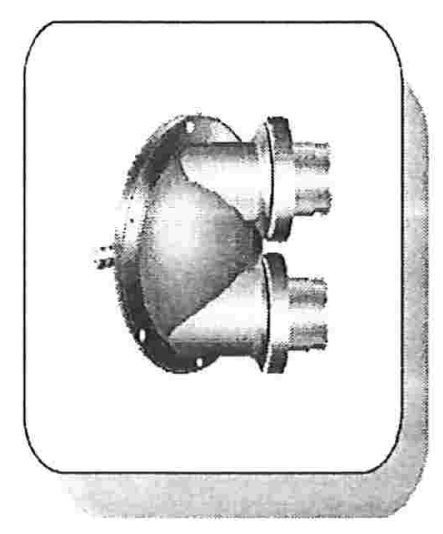

Figura 1.3: Unidade de bombeamento do DAV paracorpóreo desenvolvido no Instituto do Coração do Hospital das Clínicas da Faculdade de Medicina da Universidade de São Paulo. 
O uso deste dispositivo é mostrado na Figura 1.4.

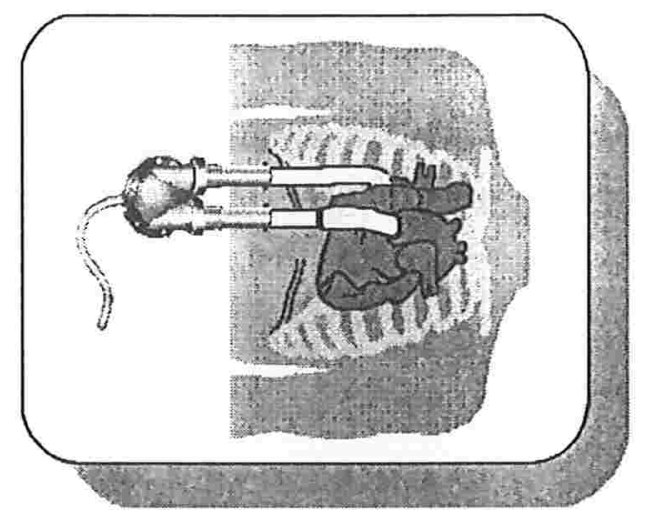

Figura 1.4: Uso do DAV paracorpóreo desenvolvido no InCor.

Com relação aos DAV's surgem algumas questões: ${ }^{1}$ Como ondulações e deformações no tubo interfeririam com o escoamento sangüíneo? Seria possível tirar vantagem dessas deformações? Nesse caso, onde elas deveriam estar localizadas? Qual seria o melhor formato para a secção transversal?

Embora esses e outros problemas possam ser analisados usando-se modelos tridimensionais de tubos flexíveis, como o de Rosar [18] que estudou em que condições eles se colapsam, aqui serão apresentados somente resultados relativos a modelos bidimensionais, a título de simplificação dos modelos e evitar as grandes dificuldades introduzidas por cálculos em três dimensões.

Este e tantos outros problemas oriundos da Medicina e da Biologia caracterizam-se por apresentarem interações entre fluidos incompressíveis (exemplo, sangue) e interfaces elásticas (exemplo, tubos flexíveis, músculos, etc.). Neste trabalho, objetiva-se apresentar, implementar e testar adequadamente alguns dos elementos necessários à modelagem matemática e à simulação numérica de problemas como os caracterizados acima, tendo sempre como motivação científica o problema de escoamento incompressível em tubos flexíveis. A ferramenta básica empregada aqui será o Método da Fronteira Imersa.

\subsection{Revisão da Literatura}

Em 1972, Peskin [12] apresentou um modelo matemático e um método computacional para estudar o escoamento de sangue ao redor da válvula mitral. Em contraste com muitos outros autores que estudam o movimento dessa válvula, ele não fez nenhuma suposição geométrica. A partir das características físicas e fisiológicas do fluido, da válvula e da parede do coração e

\footnotetext{
${ }^{1}$ Segundo o Dr Affeld da Medizinische Facultät der Humboldt-Universität, Berlim.
} 
aplicando as Leis de Newton, Peskin deduziu as equações dinâmicas do movimento que descrevem a forte interação existente entre o movimento da válvula e o escoamento do sangue.

No modelo bidimensional inicial foi considerada apenas a parte do coração que inclui o átrio e ventrículo esquerdos e a válvula mitral. Além disso, foram considerados também os tendões e os músculos papilares. Para simplificar a implementação computacional do método, considerou-se que a estrutura cardíaca, completamente imersa no fluido, possui a mesma densidade do fluido. Por esta razão, o método de Peskin é conhecido como Método da Fronteira Imersa.

Na elaboração do Método da Fronteira Imersa, Peskin descreveu as variáveis do fluido e da fibra imersa de formas distintas. As variáveis do fluido (velocidade, pressão, densidade de força) são descritas na forma Euleriana, isto é, em cada ponto do espaço é analisado o comportamento de uma determinada variável ao longo do tempo. A posição da fibra está descrita na forma Lagrangiana, isto é, cada ponto material da fibra é identificado por um parâmetro que permite que se observe como varia sua posição ao longo do tempo.

Embora o modelo inicial mencionado anteriormente fosse simples, ele permitiu a visualização da formação de um vórtice atrás da válvula mitral, mostrando como são formados e que papel realizam no fechamento desta válvula sem retorno sangüíneo. Peskin [13] estendeu o modelo original incluindo uma descrição mais adequada do coração, a qual foi usada em aplicações de fisiologia e patofisiologia. Desde 1989, McQueen e Peskin [15], [14] vêm desenvolvendo um modelo tridimensional do coração que inclui várias estruturas cardíacas obtidas por intermédio de modelagem matemática.

O Método da Fronteira Imersa sofre diversas limitações (Roma [17]): estabilidade, relacionada à rigidez da fronteira elática imersa; captura de detalhes geométricos sutis da fronteira e uso de números de Reynolds não fisiológicos. O caminho adotado para evitar a restrição da estabilidade tem sido sempre a busca de uma formulação conveniente de uma forma implícita para o Método da Fronteira Imersa, porém, este problema continua sendo estudado. As duas últimas limitações podem ser entendidas como uma "perda de resolução", uma vez que não é uma limitação do método em si, mas dependem de recursos computacionais como velocidade de processamento e capacidade de armazenamento. Roma [17], motivado por este problema, desenvolveu uma implementação adaptativa do Método da Fronteira Imersa que usa uma técnica de refinamento adaptativo de malhas. Refinando uma vizinhança próxima da fibra elástica, ele conseguiu a mesma resolução que pode ser obtida se uma malha uniforme e bem fina fosse usada.

Lai [10] deduziu uma condição de estabilidade para o esquema explícito do Método da Fronteira Imersa em uma dimensão e que servirá como ponto de partida na escolha do passo no tempo em problemas de fronteira imersa em mais dimensões.

Foi desenvolvido por Aaron L. Fogelson e David J. Eyre, ambos do Departamento de Matemática da Universidade de Utah, o Immersed Boundary and Interface Software (IBIS). Este 
software, de fácil manuseio, tem como objetivo viabilizar de forma rápida as simulações de problemas de interfaces empregando o Método da Fronteira Imersa. O IBIS tem implementado o Método da Fronteira Imersa em duas dimensões, possui uma meta linguagem para especificar os parâmetros e condições iniciais requeridas pelo método e uma interface gráfica para visualização dos resultados. Na Figura 1.5, mostra-se a página principal do IBIS.

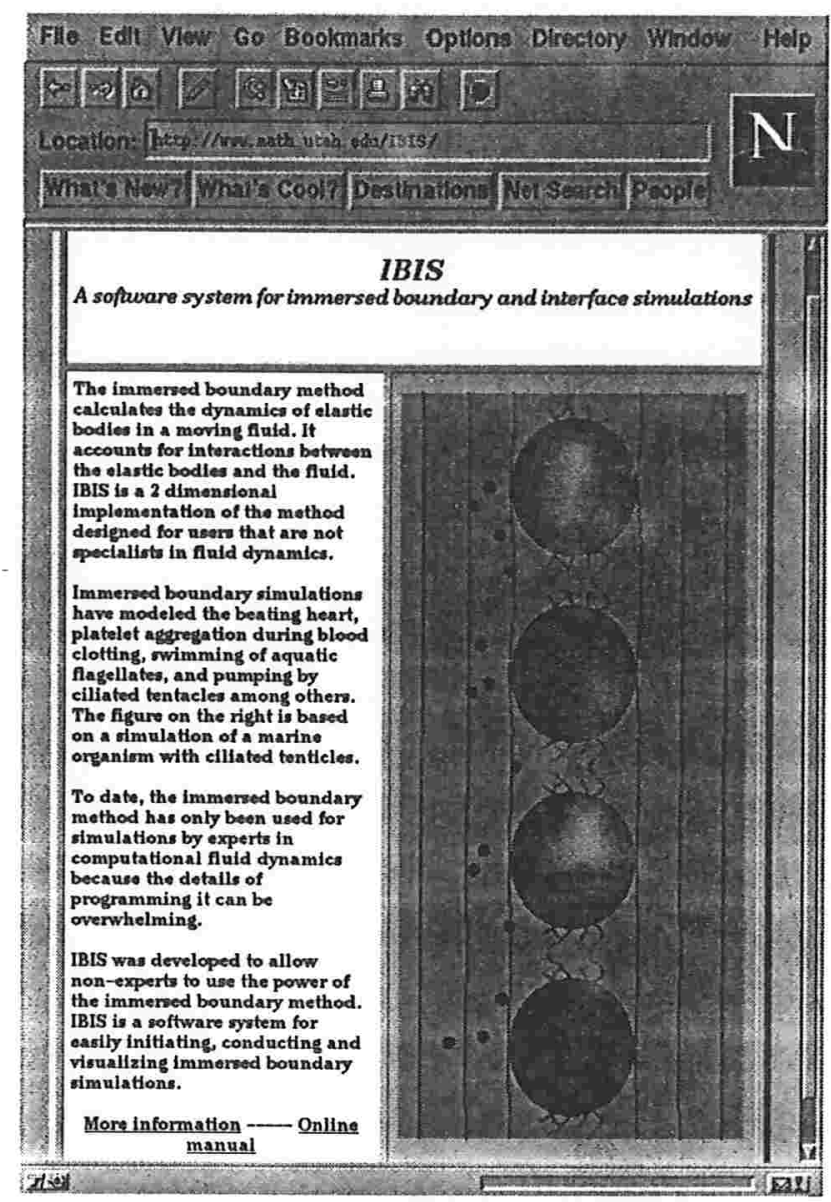

Figura 1.5: IBIS: http://www.math.utah.edu/IBIS/.

O Método da Fronteira Imersa é uma ferramenta extremamente útil de modelagem. Sua utilidade é ilustrada na simulação de uma variedade de problemas complexos. Como exemplo, é possível mencionar a agregação de plaquetas durante a coagulação sangüínea [8], a locomoção de animais aquáticos [7], a dinâmica de fluido no interior do ouvido [2], o escoamento incompressível em tubos tridimensionais colapsáveis [18], a modelagem do escoamento arteriolar e o respectivo transporte de massa [1] e a simulação de um fluido incompressível passando por um arranjo de cilindros circulares [10]. 


\subsection{Apresentação deste trabalho}

Este trabalho tem por objetivos apresentar, implementar e testar adequadamente o modelo adotado por Peskin [13] para simular a presença de fonte e sumidouro, elementos de modelagem adotados para simular a entrada e saída de fluido da região estudada. Para isto, foi utilizado como código base o código desenvolvido na linguagem C por Masaishi [21], ao qual, além de alterações para incorporar os novos elementos de modelagem, foram acrescentadas algumas subrotinas de teste usadas em sua validação. No Capítulo 2, é feita uma exposição do Método da Fronteira Imersa: sua formulação matemática, esquemas numéricos e algoritmo. No Capítulo 3, apresentase o Método da Fronteira Imersa contendo fonte e sumidouro. No Capítulo 4, são apresentados os testes numéricos utilizados para verificar a ordem do Método da Fronteira Imersa, para ilustrar o funcionamento dos modelos adotados para fonte e sumidouro e para simular o escoamento laminar entre placas paralelas. Conclusões e planos futuros são discutidos no Capítulo 5. Alguns apêndices são incluidos no fim do trabalho com o objetivo de esclarecer pontos abordados durante seu desenvolvimento. 


\section{Apresentação do Método da Fronteira Imersa}

Neste capítulo será exposto o modelo matemático e o método computacional elaborados por Peskin [13] para estudar a interação entre um fluido e fibras elásticas nele imersa. Tal interação pode ser encontrada na Biologia, Medicina, Física e Engenharia.

Na Seção 2.1, serão deduzidas as equações do modelo que governam a interação dinâmica. entre o fluido e a fibra. Tais equações foram encontradas considerando-se uma fibra imersa com propriedades elásticas, infinitamente delgada e com massa desprezível, e um fluido viscoso e incompressível contido num domínio $\Omega \subset \mathbf{R}^{2}$.

Na Seção 2.2, apresentam-se as notações e as discretizações utilizadas para aproximar as derivadas que aparecem no modelo matemático, em particular, as aproximações para os operadores gradiente, divergente e Laplaciano.

Na Seção 2.3, apresenta-se o algoritmo do método computacional.

\subsection{Modelo matemático}

As equações do escoamento de um fluido viscoso, incompressível, no qual os efeitos de gravidade não são considerados, têm a seguinte forma:

$$
\begin{aligned}
\rho\left(\frac{\partial \mathbf{u}}{\partial t}+\mathbf{u} \cdot \nabla \mathbf{u}\right) & =-\nabla p+\mu \Delta \mathbf{u}, \\
\nabla \cdot \mathbf{u} & =0,
\end{aligned}
$$

onde as incógnitas são a função vetorial $\mathbf{u}=\mathbf{u}(\mathbf{x}, t)$ ( $\mathbf{x}$ é a posição no espaço e $t$ é o tempo), que representa a velocidade do fluido, e a função escalar $p=p(\mathbf{x}, t)$, que representa o campo de pressão; os parâmetros físicos $\rho$ e $\mu$, constantes no espaço e no tempo, são respectivamente 
a densidade de massa e o coeficiente de viscosidade. A equação (2.1) representa a conservação do momento e a equação (2.2) é a equação de continuidade. As equações (2.1)-(2.2) devem ser completadas com uma condição de fronteira adequada e condição inicial para o campo velocidade u. Será assumido aqui o domínio de trabalho $\Omega=[0,1] \times[0,1]$ e condições periódicas para a velocidade na fronteira, isto é

$$
\begin{array}{ll}
\mathbf{u}((0, y), t)=\mathbf{u}((1, y), t), & \forall t \geq 0, \\
\mathbf{u}((x, 0), t)=\mathbf{u}((x, 1), t), & \forall t \geq 0 .
\end{array}
$$

A condição inicial para a velocidade é $\mathbf{u}(\mathbf{x}, 0)=\mathbf{u}_{0}(\mathbf{x}), \mathbf{x} \in \Omega$, onde $\mathbf{u}_{0}$ é dado.

A intenção é modelar o escoamento de um fluido viscoso, incompressivel, no qual uma fibra elástica encontra-se imersa. O conjunto formado pelo fluido e pela fibra será denominado sistema fluido-fibra.

A posição da fronteira imersa será descrita na forma Lagrangeana. Dessa forma, cada ponto material da fibra é identificado por um parâmetro que permite que se observe como varia sua posição ao longo do tempo. A função que descreve a posição será $\mathrm{X}(s, t)=\left(X_{1}(s, t), X_{2}(s, t)\right)$, $s \in S=[a, b]$, onde cada valor do parâmetro $s$ identifica univocamente um ponto material da fronteira imersa.

As considerações feitas sobre a fibra são:

(i) a fibra é infinitamente delgada e tem massa desprezível;

(ii) a fibra apresenta propriedades elásticas.

Devido à consideração (i), a fibra não ocupa volume. Isso permite considerar o sistema fluido-fibra como sendo simplesmente um fluido viscoso e incompressível, no qual age uma força oriunda nos pontos onde a fibra está localizada. Em particular, o campo de velocidade $\mathbf{u}$ do sistema fluido-fibra continua satisfazendo $\nabla \cdot \mathbf{u}=0$ (condição de incompressibilidade). Devido à consideração (ii), a fibra tem uma direção preferencial para exercer força (o sistema fluido-fibra é anisotrópico).

As equações que modelam o sistema fluido-fibra são dadas por:

$$
\begin{aligned}
\rho\left(\frac{\partial \mathbf{u}}{\partial t}+\mathbf{u} \cdot \nabla \mathbf{u}\right) & =-\nabla p+\mu \Delta \mathbf{u}+\mathbf{F} \\
\nabla \cdot \mathbf{u} & =0
\end{aligned}
$$

O termo F, usualmente um campo de força externo, neste caso representa a força produto da presença da fibra elástica. Note que esta força é descontínua, diferindo de zero somente nos pontos onde a fibra está localizada. Em geral, as fibras se mexem devido a suas propriedades 
elásticas e ao movimento do fluido. Seja $\mathrm{f}(s, t)$ a densidade de força elástica aplicada pela fibra no fluido. Aplicando a segunda lei de Newton a um trecho arbitrário da fibra definido em $s \in\left[s_{1}, s_{2}\right]$ (ver Figura 2.1), obtém-se

$$
\frac{d}{d t} \int_{s_{1}}^{s_{2}} m(s) \frac{\partial \mathbf{X}(s, t)}{\partial s} d s=\left.\mathbf{T} \tau(s, t)\right|_{s_{1}} ^{s_{2}},+\int_{s_{1}}^{s_{2}}(-\mathbf{f}(s, t)) d s
$$

onde $m(s)$ representa a densidade de massa unidimensional.

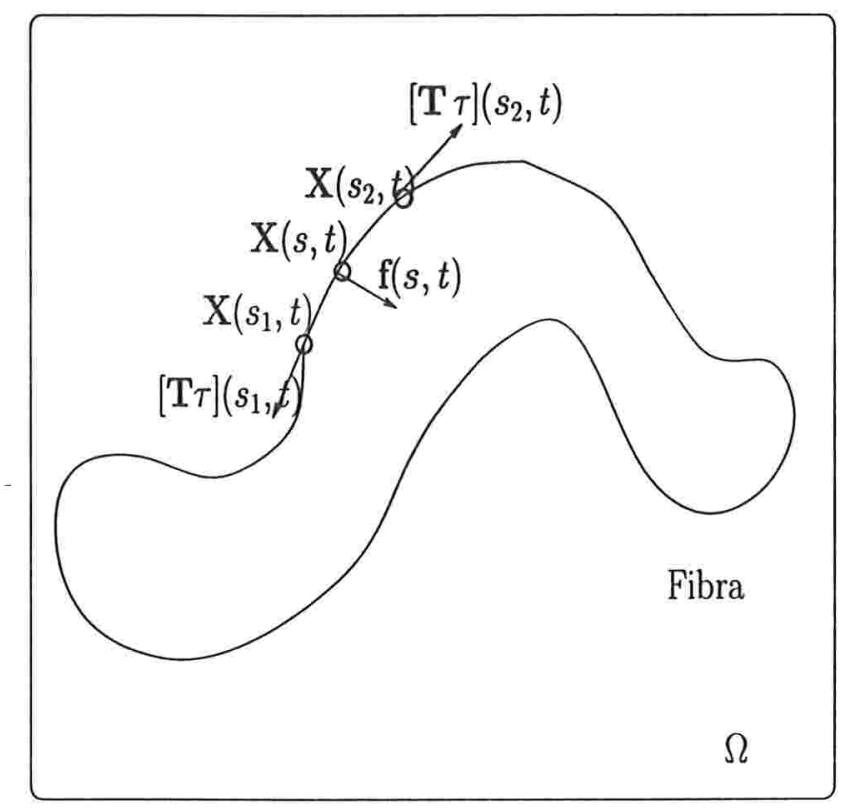

Figura 2.1: Forças atuantes na fibra no trecho $\mathbf{X}\left(s_{1}, t\right)$ e $\mathbf{X}\left(s_{2}, t\right)$.

T é a tensão na fibra cujo valor é dado pela lei geralizada de Hooke

$$
\mathbf{T}=\mathbf{T}\left(\left\|\frac{\partial \mathbf{X}}{\partial s}\right\|, s, t\right)
$$

$\tau$ é o vetor tangente unitário,

$$
\tau(s, t)=\frac{\frac{\partial \mathbf{X}(s, t)}{\partial s}}{\left\|\frac{\partial \mathbf{X}(s, t)}{\partial s}\right\|},
$$

e (-f) é a reação do fluido em resposta à ação da fibra sobre ele. Assumindo-se que a fibra possui densidade de massa desprezível, o primeiro membro da equação (2.5) se anula. Aplicando-se o Teorema Fundamental do Cálculo e reordenando-se os termos da equação (2.5), tem-se

$$
\int_{s_{1}}^{s_{2}} \mathbf{f}(s, t) d s=\int_{s_{1}}^{s_{2}} \frac{\partial(T \tau)}{\partial s}(s, t)
$$


Como $s_{1}$ e $s_{2}$ são arbitrários (o segmento de fibra é arbitrário), conclui-se da equação (2.7) que a densidade de força elástica é dada por

$$
\begin{aligned}
\mathbf{f}(s, t)=\frac{\partial \mathbf{T} \tau}{\partial s}(s, t) & =\frac{\partial \mathbf{T}}{\partial s} \tau+\mathbf{T} \frac{\partial \tau}{\partial s} \\
& =\frac{\partial \mathbf{T}}{\partial s} \tau+\mathbf{T}\left\|\frac{\partial \mathbf{X}}{\partial s}\right\| K \mathbf{n}
\end{aligned}
$$

onde $K=\left\|\frac{\partial \tau}{\partial s}\right\| /\left\|\frac{\partial \mathbf{X}}{\partial s}\right\|$ é a curvatura da fibra e $\mathbf{n}=\frac{\partial \tau}{\partial s} /\left\|\frac{\partial \tau}{\partial s}\right\|$, o vetor normal a ela. Para encontrar a relação entre a densidade de força elástica e a densidade de força das equações do fluido, considere uma região arbitrária $\mathbf{R}$ do domínio $\Omega$ como mostrado na Figura 2.2.

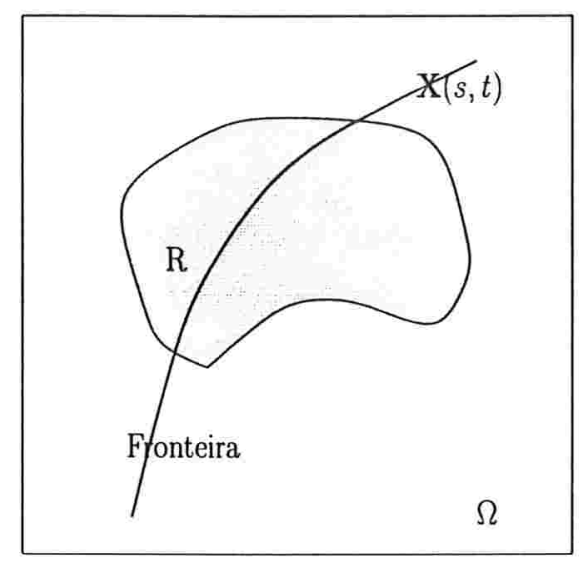

Figura 2.2: $\mathrm{R}$ é uma região arbitrária de domínio $\Omega$.

A força total na região $R$ é dada por

$$
\begin{aligned}
\int_{R} \mathbf{F}(\mathbf{x}, t) d \mathbf{x} & =\int_{\{s \in S \mid \mathbf{X}(s, t) \in R\}} \mathbf{f}(s, t) d s \\
& =\int_{S} \mathbf{f}(s, t) W_{R}(\mathbf{X}(s, t)) d s \\
& =\int_{S} \mathbf{f}(s, t)\left(\int_{R} \delta(\mathbf{x}-\mathbf{X}(s, t)) d \mathbf{x}\right) d s \\
& =\int_{R}\left(\int_{S} \delta(\mathbf{x}-\mathbf{X}(s, t)) \mathbf{f}(s, t) d s\right) d \mathbf{x}
\end{aligned}
$$

onde $W_{R}(\mathbf{x})=1$ se $\mathbf{x} \in R$ e 0 caso contrário, $\delta$ é a "função" delta de Dirac ${ }^{1}$ bidimensional. Como R é arbitrário, então

$$
\mathbf{F}(\mathbf{x}, t)=\int_{S} \mathbf{f}(s, t) \delta(\mathbf{x}-\mathbf{X}(s, t)) d s .
$$

\footnotetext{
${ }^{1}$ Para maiores detalhes ver Apêndice A.
} 
Devido à viscocidade do fluido, os pontos da fibra se movimentam com a velocidade local do fluido (condição no-slip). Esta condição pode ser expressa matematicamente em termos do campo de velocidade do fluido como

$$
\frac{\partial \mathbf{X}(s, t)}{\partial t}=\mathbf{u}(\mathbf{X}(s, t), t)
$$

Usando-se as propriedades da função delta de Dirac, é possível se expressar o lado direito da equação (2.11) em termos do campo de velocidades definido nos pontos $\mathrm{x} \in \Omega$, isto é,

$$
\mathbf{u}(\mathbf{X}(s, t), t)=\int_{\Omega} \mathbf{u}(\mathbf{x}, t) \delta(\mathbf{x}-\mathbf{X}(s, t)) d \mathbf{x} .
$$

Assim, (2.11) pode ser escrito como

$$
\frac{\partial \mathbf{X}(s, t)}{\partial t}=\int_{\Omega} \mathbf{u}(\mathbf{x}, t) \delta(\mathbf{x}-\mathbf{X}(s, t)) d \mathbf{x}
$$

\subsubsection{Resumo das equações do modelo matemático}

Das considerações anteriores, o conjunto de equações que modelam a interação de um fluido incompressível com viscocidade e densidade constantes com uma fibra elástica, infinitamente delgada e de massa desprezível, são dadas por

$$
\begin{aligned}
\rho\left(\frac{\partial \mathbf{u}}{\partial t}+\mathbf{u} \cdot \nabla \mathbf{u}\right) & =-\nabla p+\mu \Delta \mathbf{u}+\mathbf{F} \\
\nabla \cdot \mathbf{u} & =0
\end{aligned}
$$

onde,

$$
\begin{aligned}
\mathbf{F}(\mathbf{x}, t) & =\int_{S} \mathbf{f}(s, t) \delta(\mathbf{x}-\mathbf{X}(s, t)) d s \\
\mathbf{f}(s, t) & =\frac{\partial \mathbf{T} \tau}{\partial s}(s, t)
\end{aligned}
$$

com

$$
\frac{\partial \mathbf{X}(s, t)}{\partial t}=\int_{\Omega} \mathbf{u}(\mathbf{x}, t) \delta(\mathbf{x}-\mathbf{X}(s, t)) d \mathbf{x}
$$

As funções incógnitas são o campo velocidade, $\mathbf{u}=(u, v)$, o campo pressão $p$ e a posição da fibra, X. Estas equações fornecem uma formulação mista Euler-Lagrangeana do problema. As quantidades associadas ao fluido (velocidade, pressão e densidade de força) são descritas na forma Euleriana. A posição da fibra é descrita na forma Lagrangeana. As equações (2.13)-(2.14) são as equações de Navier-Stokes para um fluido viscoso, incompressível, com densidade de massa constante, afetado pelo movimento de uma fibra elástica imersa. As 
equações (2.15) e (2.17) descrevem a interação entre fluido e fibra. A equação da fronteira, (2.16), fornece a relação existente entre a densidade de força elástica e tensão. Note que o delta de Dirac é empregado para passar da formulação Euleriana para a Lagrangeana e vice-versa.

Como será visto a seguir, a formulação apresentada inspira um método computacional para resolver simultaneamente as equações (2.13)-(2.17).

Outra forma de se expressar estas equações é

$$
\begin{aligned}
\rho\left(\frac{\partial \mathbf{u}}{\partial t}+\mathbf{u} \cdot \nabla \mathbf{u}\right) & =-\nabla p+\mu \Delta \mathbf{u}+\int_{S} \frac{\partial \mathrm{T} \tau}{\partial s}(s, t) \delta(\mathbf{x}-\mathbf{X}(s, t)) d s \\
\nabla \cdot \mathbf{u} & =0 \\
\frac{\partial \mathbf{X}(s, t)}{\partial t} & =\int_{\Omega} \mathbf{u}(\mathbf{x}, t) \delta(\mathbf{x}-\mathbf{X}(s, t)) d \mathbf{x}
\end{aligned}
$$

denominada, formulação função delta (Stockie [19]).

\subsection{Método computacional}

Um método computacional para resolver as equações (2.13)-(2.17) pode ser formulado empregandose diferenças finitas. O domínio $\Omega=[0,1] \times[0,1]$ será dividido em uma malha computacional uniforme de tamanho $N \times N$, onde $\mathbf{x}_{i, j}=\left(x_{i}, y_{j}\right)=(i h, j h)$, tendo espaçamento $h=\frac{1}{N}$ em ambas direções para simplificar as discretizações (malha Euleriana). As quantidades $\mathbf{u}$ e $p$ são periódicas na fronteira deste domínio, isto é, tem o mesmo valor em $\left(x_{0}, y_{i}\right)$ e $\left(x_{N}, y_{i}\right)$ assim como em $\left(x_{i}, y_{0}\right)$ e $\left(x_{i}, y_{N}\right)$. A configuração inicial da fronteira será discretizada em um conjunto de $N_{b}$ pontos (malha Lagrangeana). Se a fronteira for dada por uma curva fechada, então, $\mathbf{x}\left(s_{0}, t\right)=\mathbf{x}\left(s_{N_{B}}, t\right), \forall t \geq 0$, onde $s_{l}=a+l \Delta s$ e $\Delta s=\frac{b-a}{N_{b}}\left(s_{0}=a, s_{N_{B}}=b\right)$.

As quantidades são procuradas nos tempos $t_{n}=n \Delta t$, onde $\Delta t$ é o passo no tempo. A Figura 2.3 mostra malhas típicas a serem empregadas.

\subsubsection{Notações e operadores de diferença empregados}

As seguintes notações serão usadas para as aproximações dos campos velocidade, pressão e densidade de força:

$$
\begin{aligned}
\mathbf{u}_{i, j}^{n} & \approx \mathbf{u}\left(x_{i}, y_{j}, t_{n}\right) \\
p_{i, j}^{n} & \approx p\left(x_{i}, y_{j}, t_{n}\right), \quad i, j=0,1, \ldots, N-1, \\
\mathbf{F}_{i, j}^{n} & \approx \mathbf{F}\left(x_{i}, y_{j}, t_{n}\right) .
\end{aligned}
$$


Para a localização da fibra e densidade de força elástica, tem-se:

$$
\begin{aligned}
\mathrm{X}_{l}^{n} & \approx \mathrm{X}\left(s_{l}, t_{n}\right), \\
\mathbf{f}_{l}^{n} & \approx \mathrm{f}\left(s_{l}, t_{n}\right) \quad l=0,1, \ldots, N_{b}-1 \text { e } n=0,1, \ldots
\end{aligned}
$$

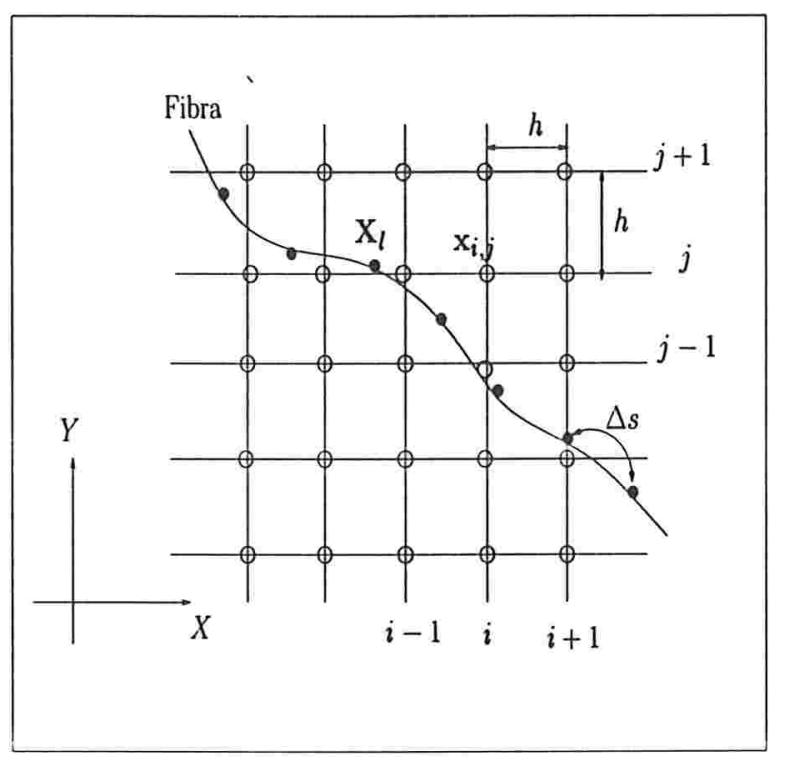

Figura 2.3: Malhas Euleriana ("o") e Lagrangeana ("๑").

Os operadores de diferenças finitas usados aqui foram baseados na discretização proposta por Printz e Peskin [16]:

- para aproximar as primeiras derivadas no espaço serão empregadas as aproximações para frente, para trás e centrada, dadas respectivamente por

$$
\begin{aligned}
D_{x}^{+} \phi_{i, j} & =\frac{\phi_{i+1, j}-\phi_{i, j}}{h}, \\
D_{x}^{-} \phi_{i, j} & =\frac{\phi_{i, j}-\phi_{i-1, j}}{h}, \\
D_{x}^{0} \phi_{i, j} & =\frac{\phi_{i+1, j}-\phi_{i-1, j}}{2 h},
\end{aligned}
$$

sendo (2.18)-(2.19) de primeira ordem e a última, (2.20), de segunda ordem. Observe que definições similares serão usadas para a derivada $y, D_{y}^{+}, D_{y}^{-}$e $D_{y}^{0}$;

- para aproximar os operadores diferenciais gradiente, divergente e Laplaciano, serão utilizadas aproximações de segunda ordem dadas, respectivamente, por

$$
(G \phi)_{i, j}=\left(D_{x}^{0}, D_{y}^{0}\right) \phi_{i, j}
$$




$$
\begin{aligned}
\mathrm{D} \cdot \mathbf{u}_{i, j} & =D_{x}^{0} u_{i, j}+D_{y}^{0} v_{i, j} \\
\Delta_{h} \phi_{i, j} & =\left(D_{x}^{+} D_{x}^{-}+D_{y}^{+} D_{y}^{+}\right) \phi_{i, j} .
\end{aligned}
$$

A aplicação do operador divergente $\mathbf{D}$ ao operador $G$ resulta em um operador de diferenças para o Laplaciano conhecido como a "regra dos cinco pontos":

$$
\mathrm{D} \cdot G \phi_{i, j}=\frac{\phi_{i+2 . j}+\phi_{i-2, j}+\phi_{i, j+2}+\phi_{i, j-2}-4 \phi_{i, j}}{4 h^{2}} .
$$

Da mesma forma, pode-se empregar aproximações para uma quantidade definida sobre a fibra, definindo

$$
\begin{aligned}
& D_{s}^{+} \Psi_{l}=\frac{\Psi_{l+1}-\Psi_{l}}{\Delta s}, \\
& D_{s}^{-} \Psi_{l}=\frac{\Psi_{l}-\Psi_{l-1}}{\Delta s} .
\end{aligned}
$$

A função delta de Dirac bidimensional que aparece nas equações (2.15) e (2.17) é aproximada por uma função delta discreta, $\delta_{h}$, mostrada na Figura 2.4, a qual é o produto de duas funções discretas unidimensionais,

$$
\delta_{h}\left(x_{i}, y_{j}\right)=d_{h}\left(x_{i}\right) \cdot d_{h}\left(y_{j}\right)
$$

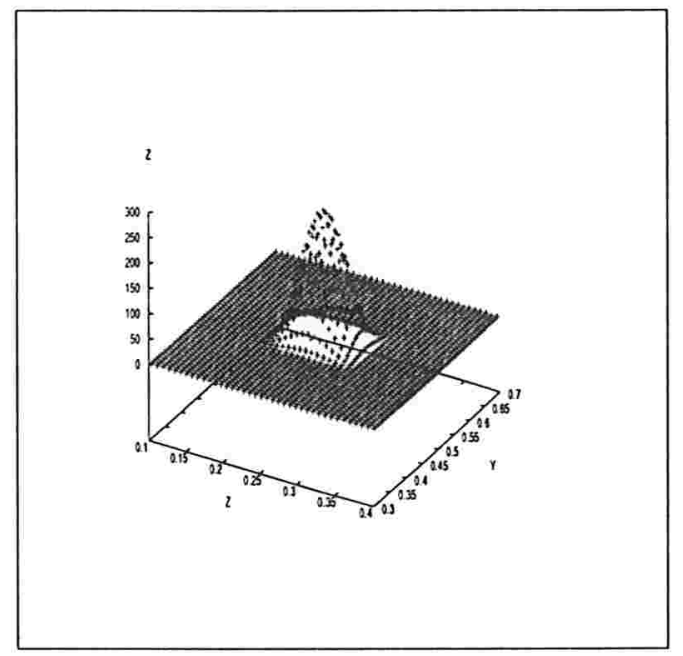

Figura 2.4: Gráfico da função discreta delta de Dirac bidimensional, $\delta_{h}\left(\mathrm{x}-\mathrm{x}_{0}\right)$, com centro no ponto $\mathrm{x}_{0}=(0.25,0.5)\left(h=\frac{1}{n}, n=64\right)$.

Uma possível escolha para a função $d_{h}$ usada no Método da Fronteira Imersa é

$$
d_{h}(x)=\left\{\begin{aligned}
\frac{1}{4 h}\left(1+\cos \left(\frac{\Pi x}{2 h}\right)\right), & |x|<2 h \\
0, & |x| \geq 2 h
\end{aligned}\right.
$$


cujo gráfico está mostrado na Figura 2.5. Esta seleção particular da aproximação da função delta de Dirac é motivada por um conjunto de propriedades de compatibilidade discretas que foram descritas por Peskin [13]. Estas propriedades e outras aproximações para o delta de Dirac serão apresentadas no Apêndice A.

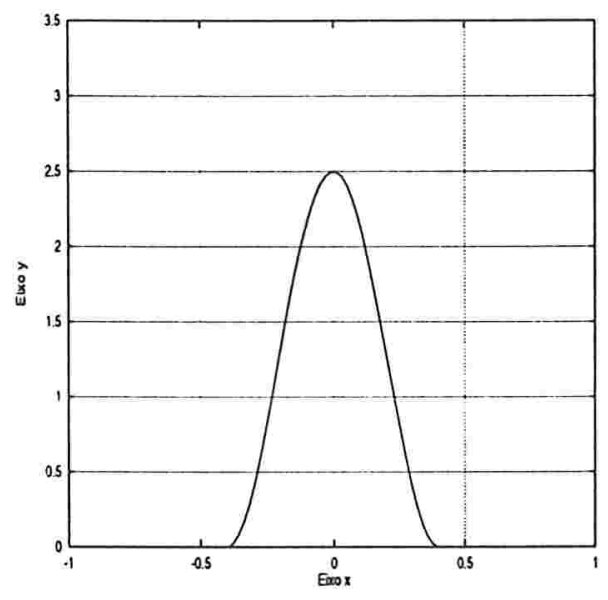

Figura 2.5: Gráfico da função discreta delta de Dirac unidimensional, $d_{h}(x)\left(h=\frac{1}{n}, n=5\right)$.

\subsubsection{Discretização e resolução numérica do modelo matemático}

As equações do movimento (2.13)-(2.17) podem ser particionadas em três grupos: equações do fluido (2.13)-(2.14), equações de interação (2.15) e (2.17), e equação da fibra (2.16). Essa partição se reflete no método computacional.

As equações do fluido (equações de Navier Stokes) serão resolvidas por um método de projeção, que é uma variação do Método de Projeção de Chorin [4]. Ao contrário do caso compressível, a pressão em escoamentos incompressíveis não tem significado termodinâmico, sua função é a de forçar a incompressibilidade (2.14). A idéia central do método de projeção é livrar as equações do fluido do termo gradiente de pressão através da introdução de um operador que projeta um campo de velocidade no espaço vetorial dos campos de livre-divergência (campo vetorial com divergência zero). Por trás do operador de projeção está o Teorema de Decomposição de Hodge-Helmholtz que se escreve, em sua versão periódica, como

Teorema 1 Qualquer campo vetorial periódico $\mathrm{w}$ definido em um retângulo $\Omega$ pode ser unicamente decomposto na forma

$$
\mathbf{w}=\mathbf{u}^{d}+\nabla \Phi
$$

onde $\mathbf{u}^{d}$ é um campo vetorial periódico tal que $\nabla \cdot \mathbf{u}^{d}=0$ e $\Phi$ uma função escalar periódica. 
A unicidade da função escalar $\Phi$ deve ser entendida " a menos de uma constante". A prova desse teorema pode ser encontrada no Apêndice B. Uma versão para domínios não periódicos é dada por Chorin e Marsden [5].

Algumas observações:

- é possível mostrar que com o produto interno usual definido em $\mathrm{L}^{2}(\Omega)$,

$$
<\mathbf{u}, \mathbf{v}>=\int_{\Omega}\left(\sum_{1}^{2} u_{i} . v_{i}\right) d \mathbf{x}, \quad \mathbf{u}, \mathbf{v}: \mathbf{R}^{2} \rightarrow \mathbf{R}^{2},
$$

$\mathbf{u}^{d}$ é ortogonal ao gradiente da função $\Phi$, isto é, $\left\langle\mathbf{u}^{d}, \nabla \Phi\right\rangle=0$;

- o operador de projeção $\mathcal{P}$ associado a esta decomposição projeta ortogonalmente os campos vetoriais periódicos $\mathbf{w} \in \mathbf{L}^{2}(\Omega)$ no subespaço de vetores com livre-divergência;

- em termos do operador de projeção $\mathcal{P}$, as equações do fluido (2.13)-(2.14) podem ser escritas como

$$
\mathbf{u}_{t}=\mathcal{P}\left(\frac{\mu}{\rho} \Delta \mathbf{u}-(\mathbf{u} \cdot \nabla) \mathbf{u}+\frac{\mathbf{F}}{\rho}\right)
$$

que pode ser interpretada como uma equação de evolução para a velocidade dentro do espaço de campo velocidade de livre-divergência.

As equações de interação podem ser discretizadas de diversas formas. Nesse trabalho foram adotadas as discretizações

$$
\begin{aligned}
\mathbf{F}_{i, j}^{n} & =\sum_{l} \mathbf{f}_{l}^{n} \delta_{h}\left(\mathbf{x}_{i, j}-\mathbf{X}_{l}^{n}\right) \Delta s \\
\mathbf{X}_{l}^{n+1} & =\mathbf{X}_{l}^{n}+\Delta t \sum_{i, j} \mathbf{u}_{i, j}^{n+1} \delta_{h}\left(\mathbf{x}_{i, j}-\mathbf{X}_{l}^{n}\right) h^{2}
\end{aligned}
$$

para (2.15) e (2.17), respectivamente.

Embora as discretizações do delta de Dirac em (2.22)-(2.23) não precisem ser as mesmas, quando elas forem iguais valerá o análogo discreto da identidade

$$
\begin{aligned}
\int_{S} \mathbf{f}(s, t) \frac{\partial \mathbf{X}(s, t)}{\partial t} d s & =\int_{S} \mathbf{f}(s, t) \int_{\Omega} \mathbf{u}(\mathbf{x}, t) \delta(\mathbf{x}-\mathbf{X}(s, t)) d \mathbf{x} d s \\
& =\int_{\Omega} \mathbf{u}(\mathbf{x}, t) \int_{S} \mathbf{f}(s, t) \delta(\mathbf{x}-\mathbf{X}(s, t)) d s d \mathbf{x}=\int_{\Omega} \mathbf{u}(\mathbf{x}, t) \mathbf{F}(\mathbf{x}, t) d \mathbf{x}
\end{aligned}
$$

que significa que a parcela de potência $\int_{S} \mathrm{f}(s, t) \frac{\partial \mathrm{X}(s, t)}{\partial t} d s$, oriunda da ação da força elástica, é transferida integralmente para o fluido, isto é, $\int_{\Omega} \mathbf{u}(\mathbf{x}, t) \mathbf{F}(\mathbf{x}, t) d \mathbf{x}$. 
A aproximação da função delta é usada para interpolar forças e velocidades entre pontos do fluido e da fibra em uma vizinhança de tamanho $4 h \times 4 h$ : esta distribui forças da fibra para os pontos do fluido, e calcula a velocidade para os pontos da fibra a partir das velocidades vizinhas definidas nos pontos do fluido. Por exemplo, observe-se na Figura 2.6, a força fornecida pelo ponto da fibra $\mathrm{X}_{l}$ ao fluido é dado nos pontos da malha que estão dentro do quadrado de tamanho $4 h \times 4 h$ com centro nele; da mesma forma são estes pontos do fluido usados para interpolar a velocidade no ponto da fibra.

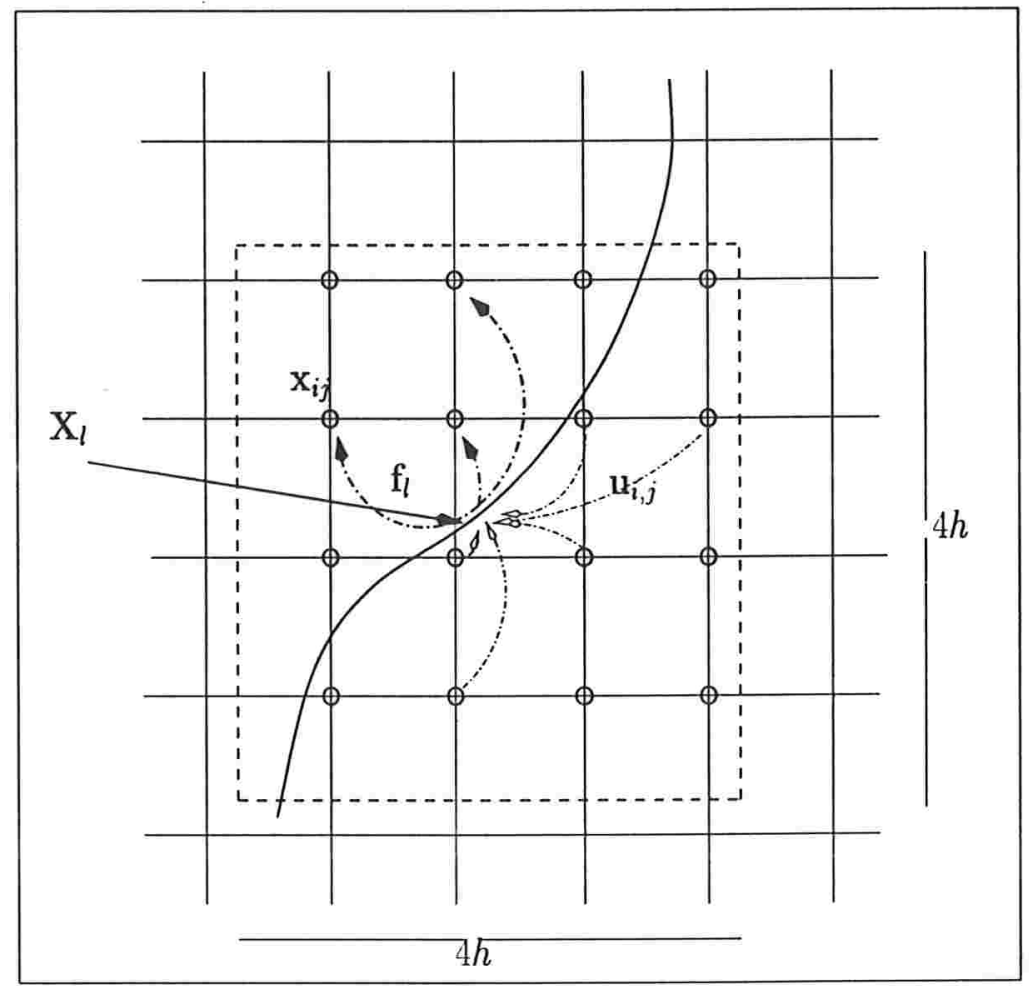

Figura 2.6: Uso da aproximação do delta de Dirac. 


\subsection{Algoritmo do Método da Fronteira Imersa}

O objetivo é calcular $\left(\mathbf{u}^{n+1}, \mathrm{X}^{n+1}\right)$ dado $\left(\mathbf{u}^{n}, \mathrm{X}^{n}\right)$.

Para isto, faz-se:

Passo 1: Calcular a densidade de força elástica $\mathbf{f}^{n}$ (dependente do modelo).

Passo 2: Distribuir a densidade de força elástica aos pontos na malha do fluido por meio da equação (2.22).

Passo 3 Passo de projeção: resolver as equações de Navier-Stokes usando uma variação do Método de Projeção de Chorin, em duas etapas:

Passo 3A: Calcular um campo de velocidade auxiliar $\mathbf{u}^{n+1,2}$ usando o esquema dado pelo algoritmo de Printz [16]

$$
\begin{aligned}
\rho\left[\frac{\mathbf{u}^{n+1,0}-\mathbf{u}^{n}}{\Delta t}\right] & =\mathbf{F}^{n}, \\
\rho\left[\frac{\mathbf{u}^{n+1,1}-\mathbf{u}^{n+1,0}}{\Delta t}+u^{n} D_{x}^{0} \mathbf{u}^{n+1,1}\right] & =\mu D_{x}^{+} D_{x}^{-} \mathbf{u}^{n+1,1}, \\
\rho\left[\frac{\mathbf{u}^{n+1,2}-\mathbf{u}^{n+1,1}}{\Delta t}+v^{n} D_{y}^{0} \mathbf{u}^{n+1,2}\right] & =\mu D_{y}^{+} D_{y}^{-} \mathbf{u}^{n+1,2},
\end{aligned}
$$

onde $\mathbf{u}^{m}=\left(u^{m}, v^{m}\right)$.

Passo 3B: Decompor a velocidade auxiliar $\mathbf{u}^{n+1,2}$ no campo de velocidades com divergente zero, $\mathbf{u}^{n+1}$, e no gradiente de um escalar $p^{n+1}$

$$
\begin{aligned}
\mathbf{u}^{n+1,2} & =\mathbf{u}^{n+1}+\frac{\Delta t}{\rho} G p^{n+1} \\
\text { D. } \mathbf{u}^{n+1} & =0 .
\end{aligned}
$$

Passo 4: Atualizar a posição da fronteira imersa usando (2.23). 
No Passo 3A, a equação (2.24) é uma fórmula explícita para $\mathbf{u}^{n+1,0}$, enquanto as duas outras equações são sistemas tridiagonais "periódicos" para as velocidades $\mathbf{u}^{n+1,1}$ e $\mathbf{u}^{n+1,2}$.

No Passo 3B, aplicando-se o operador discreto D a (2.27), temos a equação de Poisson com condições de contorno periódicas para a pressão

$$
\frac{\Delta t}{\rho} \mathrm{D} \cdot G p^{n+1}=\mathrm{D} \cdot \mathbf{u}^{n+1,2} \text {. }
$$

Calculada a pressão $p^{n+1}$, calcula-se a velocidade $\mathbf{u}^{n+1}$ de (2.27). Todo este processo pode ser escrito mais compactamente como

$$
\mathbf{u}_{i, j}^{n+1}=\mathcal{P}_{h}\left(\mathbf{u}^{n+1,2}\right)
$$

onde $\mathcal{P}_{h}$ expressa o operador de projeção ortogonal discreto. Desde que o domínio é periódico em $x$ e em $y$ e $h$ é o mesmo em cada direção, uma escolha natural para resolver a equação de Poisson é a transformada rápida de Fourier (http://theory.lcs.mit.edu/ fftw). 


\section{Modelagem de entrada e de saída de fluido no problema}

Uma forma de modelar matematicamente a entrada e a saída de fluido em um problema pode ser feita por intermédio da utilização de fontes e sumidouros. Peskin [13], simulou o fluxo do sangue no lado esquerdo do coração durante a diástole ventricular e o início da sístole. Para simular o retorno do sangue no átrio esquerdo, colocou uma fonte em sua região central e um sumidouro de mesma capacidade na parte externa do coração para absorver o volume de fluido deslocado.

No que segue, far-se-á uma apresentação do modelo adotado por Peskin para simular a presença de fontes e sumidouros e como incluir estes novos elementos de modelagem no Método da Fronteira Imersa.

$\mathrm{Na}$ Seção 3.1, apresentam-se o escoamento laminar entre placas paralelas fixas e infinitas de um fluido viscoso e incompressível em regime permanente, assim como a expressão dada para a vazão (Lei de Poiseuille), a qual será adotada neste trabalho.

Na Seção 3.2, apresentam-se o modelo que será utilizado para simular a presença de uma fonte e de um sumidouro, assim como a modificação e a solução do passo de decomposição do Método da Fronteira Imersa (Passo 3B do algoritmo apresentado na Seção 2.3).

Na Seção 3.3, faz-se uma generalização do modelo adotado na seção anterior para o caso de $k$ fontes e $k$ sumidouros e são apresentadas também, para este caso, as modificações necessárias no Método da Fronteira Imersa. 


\subsection{Escoamento entre placas paralelas e modelagem da vazão}

A modelagem da entrada e saída de fluido no domínio de definição do problema, a ser introduzida na Seção 3.2, exigirá o conhecimento da vazão de fluido em determinadas regiões do domínio.

A seguir, será visto um problema para o qual se conhece a solução exata e a qual motivará a modelagem da vazão utilizada mais adiante: o escoamento entre placas paralelas.

A vazão, definida como o volume de fluido por unidade de tempo que atravessa uma superfície S, é dada por

$$
Q=\int_{\mathbf{S}} \mathbf{u} \cdot \mathbf{n} d s
$$

onde $\mathbf{u}, \mathbf{n}$ e $d s$ são respectivamente a velocidade do fluido, a normal unitária e o elemento de área.

\subsubsection{Escoamento entre placas paralelas}

Considere o escoamento laminar ${ }^{1}$ de um fluido viscoso incompressível em regime permanente ${ }^{2}$ entre duas placas planas e paralelas, fixas e infinitas, separadas por uma distância de valor $2 a$.

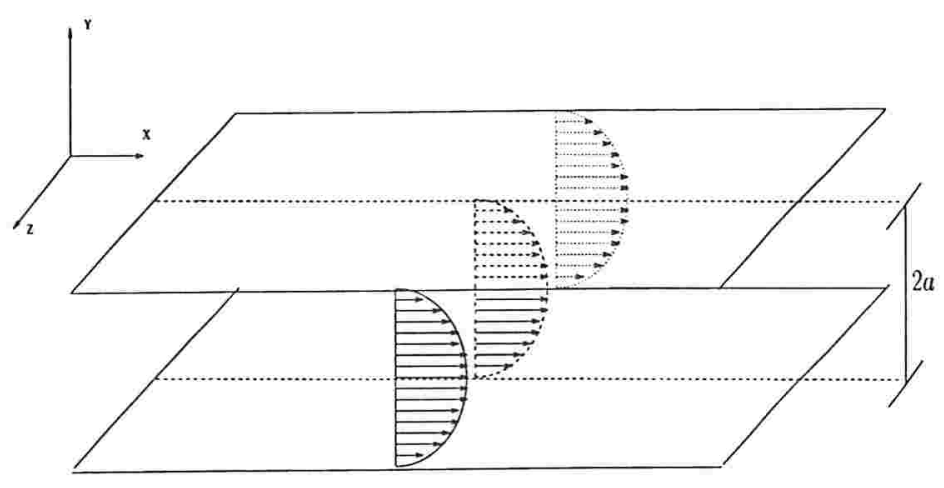

(a)

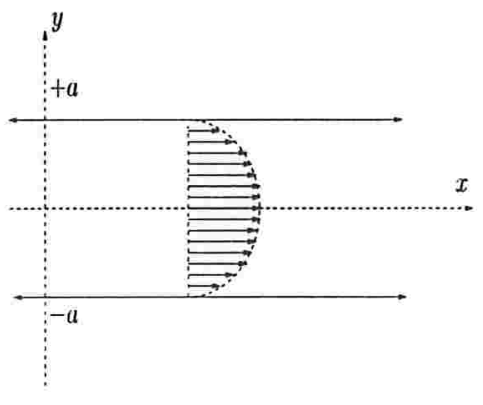

(b)

Figura 3.1: (a) Escoamento laminar entre duas placas paralelas movido pelo gradiente de pressão; (b) perfil do escoamento em um plano paralelo ao plano $y x$.

Se este escoamento é devido unicamente ao gradiente de pressão, com $p=p(x)$ e $\partial p / \partial x=$ $C<0, C$ constante, o escoamento ocorrerá no sentido positivo do eixo $x$ como se mostra

\footnotetext{
${ }^{1}$ No regime laminar, a estrutura do escoamento é caracterizada pelo movimento em lâminas ou camadas, não há mistura macroscópica de camadas de fluido adjacentes.

${ }^{2}$ Regime no qual as propriedades do fluido e as características do escoamento, em cada posição do espaço, permanecem invariáveis com o tempo.
} 
na Figura 3.1-(a). Além disso, o campo de velocidade satisfará $\mathrm{U}(x, y, z)=(u(y), 0,0)$, com condição de contorno $\mathrm{U}(x, y, z)=(0,0,0)$ em $y=a$ e $y=-a$ e $\forall x, z \in \mathbf{R}$.

O perfil de velocidades deste escoamento é o mesmo para qualquer plano paralelo ao plano $x y$ (Figura 3.1-(b)). Para deduzir a solução exata deste problema, considere as equações de Navier-Stokes escritas num destes planos

$$
\begin{aligned}
\rho\left(\frac{\partial}{\partial t} u+u \frac{\partial}{\partial x} u+v \frac{\partial}{\partial y} u\right) & =-\frac{\partial}{\partial x} p+\mu\left(\frac{\partial^{2}}{\partial x^{2}} u+\frac{\partial^{2}}{\partial y^{2}} u\right) \\
\rho\left(\frac{\partial}{\partial t} v+u \frac{\partial}{\partial x} v+v \frac{\partial}{\partial y} v\right) & =-\frac{\partial}{\partial y} p+\mu\left(\frac{\partial^{2}}{\partial x^{2}} v+\frac{\partial^{2}}{\partial y^{2}} v\right) \\
\frac{\partial}{\partial x} u+\frac{\partial}{\partial y} v & =0 .
\end{aligned}
$$

Com as hipóteses feitas anteriormente sobre o fluido, procura-se nesse plano um campo de velocidade $\mathbf{u}=(u, 0)$ com componente $u=u(y)$, satisfazendo a $u(y)=0$ em $y=-a \mathrm{e}$ $y=a$ (condição no-slip). As equações (3.2)-(3.3) são satisfeitas por tal campo de velocidade. Simplificando-se a equação (3.1) tem-se

$$
C=\mu \Delta u=\mu \frac{\partial^{2} u}{\partial y^{2}}
$$

donde se obtém

$$
u(y)=\frac{1}{2 \mu} C y^{2}+C_{1} y+C_{2},
$$

com $C_{1}$ e $C_{2}$ constantes. Usando a condição $u(y)=0$ para $y=-a$ e $y=a$, se obtém

$$
u(y)=\frac{-C}{2 \mu}\left(a^{2}-y^{2}\right) .
$$

Observe que a velocidade tem perfil parabólico, atingindo seu máximo no centro e zero sobre as placas (Figura 3.1-(b)).

Voltando ao problema tridimensional original tem-se $\mathbf{U}=(u, 0,0)$, sendo $u$ dado por (3.4). Este tipo de escoamento é conhecido também como escoamento num canal.

\subsubsection{Modelagem de vazão}

Considere a superfície retangular paralela ao plano $y z$ cujas arestas medem $L_{z}$ e $2 a$, como se ilustra na Figura 3.2. Escrevendo-se a velocidade como $\mathrm{U}=u \mathbf{e}_{x}=u(y) \mathbf{e}_{x}$ e o elemento de área como $d s=d y d z$, o volume do fluido que atravessa $\mathbf{S}$ é dado por

$$
Q=\int_{\mathbf{S}} \mathbf{U} \cdot \mathbf{n} d s=\int_{\mathbf{S}} u(y) d y d z
$$




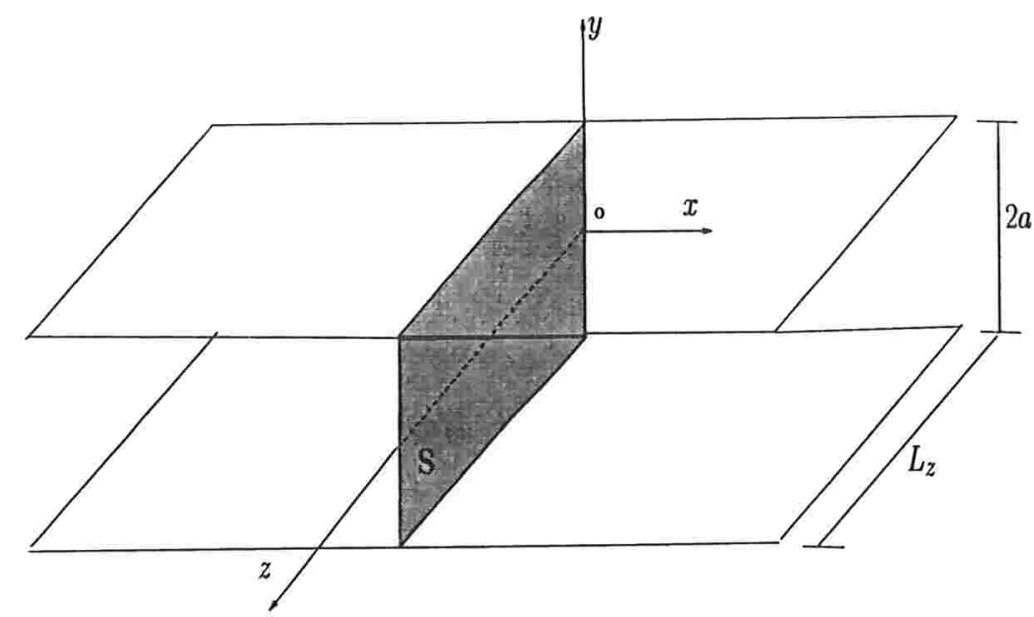

Figura 3.2: Superfície retangular paralela ao plano $y z$.

onde se utilizou $\mathbf{n}=\mathbf{e}_{\bar{x}}$. Desenvolvendo-se (3.5), obtém-se

$$
Q=\int_{0}^{L_{z}} d z \int_{-a}^{a} u(y) d y=-\frac{2 L_{z} C}{3 \mu} a^{3} .
$$

Do fato de que se tem $p=p(x)$ e $\frac{\partial p}{\partial x}=C, C$ constante, implica em

$$
p(x)=p_{1}+C x,
$$

sendo $p_{1}=p(0)$. Se $p\left(L_{x}\right)=p_{2}$, com $L_{x}>0$ e $p_{1}>p_{2}$, então

$$
C=\frac{\left(p_{2}-p_{1}\right)}{L_{x}}
$$

e a velocidade (3.4), toma a forma

$$
u(y)=-\frac{\left(p_{2}-p_{1}\right)}{2 \mu L_{x}}\left(a^{2}-y^{2}\right) .
$$

A vazão (3.6), por sua vez, pode ser escrita então como

$$
Q=-\frac{2 L_{z}\left(p_{2}-p_{1}\right)}{3 \mu L_{x}} a^{3} .
$$

Vê-se da expressão acima que a vazão é diretamente proporcional à diferença de pressão $\Delta p=p_{1}-p_{2}$, largura $L_{z}$ e ao cubo da meia altura $a$ e inversamente proporcional ao coeficiente de viscosidade e à distância $L_{x}$, que separa os pontos nos quais foram dadas as pressões ao longo do eixo $x$. 
Este resultado é análogo ao que se obtém para o escoamento de um fluido ao longo de um tubo de raio $a$ e comprimento $L$ na presença de um gradiente de pressão constante igual a $\Delta p / L$. Neste caso, a vazão é dada por

$$
Q=\frac{\pi\left(p_{1}-p_{2}\right)}{8 \mu L} a^{4},
$$

e é conhecida como Lei de Poiseuille, obtida experimentalmente por Hagen-Poiseuille entre $1839-1846$.

A forma para a vazão dada pela Lei de Poiseuille pode ser reescrita como

$$
Q=\frac{p_{1}-p_{2}}{R},
$$

onde $R=\frac{8 \mu L}{\pi a^{4}}$. Esta expressão para a vazão é conhecida como um modelo de resistência linear para a vazão, onde $p_{1}$ é a pressão de entrada e $p_{2}$ a pressão de saída, $R$ a resistência no tubo, a qual depende da viscocidade, comprimento e raio do tubo. No caso do escoamento entre placas paralelas, será entendido que a vazão também obedece um modelo de resistência linear como (3.10), para o qual $R=\frac{3 \mu L_{x}}{2 L_{z} a^{3}}$.

\subsection{Escoamentos contendo uma fonte e um sumidouro}

\subsubsection{Modelo adotado por Peskin para fonte e sumidouro}

No que se segue, apresenta-se o modelo adotado para simular as presenças de uma fonte e de um sumidouro em um domínio bidimensional $\Omega$. O modelo bidimensional pode ser considerado como uma secção longitudinal ao longo do escoamento tridimensional laminar.

As equações que regem o escoamento de um fluido viscoso contendo fonte(s) e sumidouro(s) são dadas por

$$
\begin{aligned}
\rho\left(\frac{\partial \mathbf{u}}{\partial t}+\mathbf{u} \cdot \nabla \mathbf{u}\right) & =-\nabla p+\mu \Delta \mathbf{u}+\mathbf{F} \\
\nabla \cdot \mathbf{u} & =q(\mathbf{x}, t)
\end{aligned}
$$

sendo $\mathbf{u}, p$ e $\mathbf{F}$ o campo velocidade, a pressão, e a densidade de força externa, respectivamente; $q(\mathrm{x}, t)$ é uma função escalar que modela a ação da fonte e do sumidouro. Em particular, no caso incompressível tem-se $q(\mathbf{x}, t)=0, \mathbf{x} \in \Omega, t \geq 0$.

O modelo que será apresentado para $q(\mathrm{x}, t)$ é aquele empregado por Peskin [13], para simular o fluxo do sangue no lado esquerdo do coração. Esse escoamento é influenciado pelo retorno do sangue para o átrio esquerdo pelas veias pulmonares. Para simular este retorno, Peskin colocou uma fonte no centro do átrio e um sumidouro de mesma intensidade na parte externa do coração 
para absorver o volume deslocado pelo movimento de suas paredes musculares. A forma da função $q$ adotada por ele foi

$$
q(\mathbf{x}, t)=Q(t) \Psi_{0}(\mathbf{x}),
$$

onde $Q(t)$ é a vazão do escoamento e $\Psi_{0}(\mathbf{x})$ é uma função peso que especifica a distribuição espacial da fonte e do sumidouro.

Aplicando-se o Teorema da Divergência a (3.12), decorre do fato de se ter condições periódicas para $\mathbf{u}$ em $\Omega$ que

$$
\int_{\Omega} \nabla \cdot \mathbf{u} d \mathbf{x}=\int_{\partial \Omega} \mathbf{u} \cdot \mathbf{n} d \sigma \mathbf{x}=0
$$

Substituindo-se em (3.14) as expressão dada para o divergente do campo velocidade $\mathbf{u},(3.13)$, encontra-se a condição que $\Psi_{0}(\mathrm{x})$ deve satisfazer:

$$
\int_{\Omega} \Psi_{0}(\mathbf{x}) d \mathbf{x}=0
$$

A exemplo de Peskin [13], a função $\Psi_{0}(\mathbf{x})$ a ser adotada aqui será dada por

$$
\Psi_{0}(\mathbf{x})=W_{F}(\mathbf{x})-W_{S}(\mathbf{x})
$$

onde $W_{F}$ e $W_{S}$ são duas funções não negativas com suportes compactos, cujas integrais são iguais a um.

Como $W_{F}$ e $W_{S}$ são funções peso, é natural definir a pressão na fonte e no sumidouro como

$$
\begin{aligned}
& p_{F}(t)=\int_{\Omega} p(\mathbf{x}, t) W_{F}(\mathbf{x}) d \mathbf{x}, \\
& p_{S}(t)=\int_{\Omega} p(\mathbf{x}, t) W_{S}(\mathbf{x}) d \mathbf{x},
\end{aligned}
$$

onde $p(\mathbf{x}, t)$ é a pressão no fluido. Tomando-se a pressão no sumidouro $p_{S}(t)$ como uma pressão de referência, defini-se

$$
p_{F, S}(t)=p_{F}(t)-p_{S}(t)=\int_{\Omega} p(\mathbf{x}, t) \Psi_{0}(\mathbf{x}) d \mathbf{x}=<\Psi_{0}, p>.
$$

Adota-se a vazão $Q(t)$ em termos da pressão $p_{F, S}(t)$ como sendo

$$
Q(t)=Q_{s}-\alpha_{s} p_{F, S}(t)
$$

onde $Q_{s}$ e $\alpha_{s}$ são constantes. Este é um modelo de resistência linear do fluxo do sangue pelas veias pulmonares. Esta expressão para a vazão é uma outra forma de reescrever o modelo de resistência linear (3.10) para o escoamento, onde $Q_{s}=\frac{p_{1}}{R}$ e $\alpha_{s}=\frac{1}{R}$, uma interpretação para este modelo é dada por Rosar [18]. Finalmente, a forma de função $q$ é

$$
q(\mathbf{x}, t)=\left(Q_{s}-\alpha_{s} p_{F, S}(t)\right) \Psi_{0}(\mathbf{x}) .
$$


Observe que neste modelo a vazão na fonte é dada por

$$
\int_{\text {Supp }_{F}} q(\mathrm{x}, t) d \mathbf{x}=Q(t)\left(\int_{\text {Supp }_{F}} \Psi_{0}(\mathbf{x}) d \mathbf{x}\right)=Q(t)\left(\int_{S_{u p p} W_{F}} W_{F}(\mathbf{x}) d \mathbf{x}\right)=Q(t),
$$

e a vazão no sumidouro é dada por

$$
\int_{S u p p W_{S}} q(\mathbf{x}, t) d \mathbf{x}=Q(t)\left(\int_{S u p p W_{S}} \Psi_{0}(\mathbf{x}) d \mathbf{x}\right)=Q(t)\left(\int_{S u p p W_{S}} W_{S}(\mathbf{x}) d \mathbf{x}\right)=-Q(t),
$$

onde $S u p p W_{F}$ e $S u p p W_{S}$ são os suportes compactos de $W_{F}$ e $W_{S}$, respectivamente.

A Figura 3.3 mostra a idéia de como é feito o suprimento de fluido pela fonte e sua remoção pelo sumidouro.

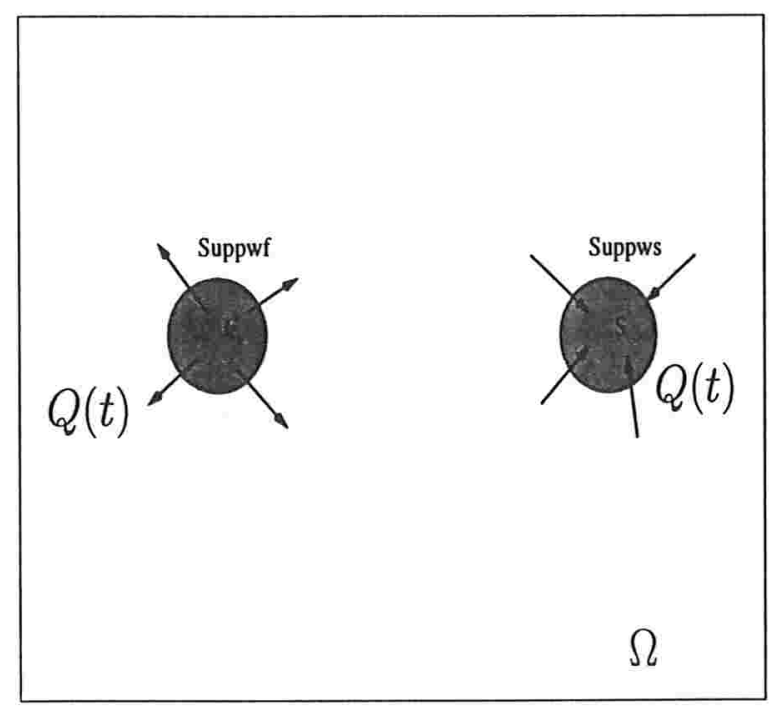

Figura 3.3: Suprimento e remoção de fluido no domínio, $\Omega$. .

\subsubsection{Inclusão de uma fonte e um sumidouro no método computacional}

A solução numérica das equações (3.11) e (3.12), com $q(\mathbf{x}, t)$ dada por (3.17) será feita pelo Método da Fronteira Imersa. Entretanto, neste caso, o passo de decomposição da velocidade auxiliar $\mathbf{u}^{n+1,2}$ (Passo $\mathbf{3 B}$ do algoritmo apresentado na Seção 2.3), verifica uma nova relação dada por

$$
\begin{aligned}
\rho\left(\frac{\mathbf{u}^{n+1}-\mathbf{u}^{n+1,2}}{\Delta t}\right) & =-G p^{n+1} \\
\mathbf{D} \cdot \mathbf{u}^{n+1} & =\left(Q_{s}-\alpha_{s} p_{F, S}^{n+1}\right) \Psi_{0}\left(\mathbf{x}_{i j}\right) .
\end{aligned}
$$


Aplicando o operador divergente discretizado, D, à equação (3.18) e usando a equação (3.19), obtém-se a equação de Poisson para a pressão

$$
\frac{\Delta t}{\rho} \mathrm{D} \cdot G p^{n+1}=\mathrm{D} \cdot \mathbf{u}^{n+1,2}-\left(Q_{s}-\alpha_{s}<\Psi_{0}, p^{n+1}>_{h}\right) \Psi_{0}\left(\mathbf{x}_{i j}\right),
$$

onde o produto interno discreto para funções escalares, $<., .>_{h}$, é definido por

$$
<\Psi_{0}, p^{n+1}>_{h}=\sum_{i, j}\left(\Psi_{0}\right)_{i, j} p_{i, j}^{n+1} h^{2} \approx \int_{\Omega} \Psi_{0}(\mathrm{x}) p^{n+1}(\mathrm{x}) d \mathbf{x} .
$$

Tomando-se $p_{0}$ como a solução da equação de Poisson

$$
\frac{\Delta t}{\rho} \mathbf{D} \cdot G p_{0}=\Psi_{0} Q_{s}
$$

e $p_{1}$ como solução da equação de Poisson

$$
\frac{\Delta t}{\rho} \mathbf{D} \cdot G p_{1}=\mathbf{D} \cdot \mathbf{u}^{n+1,2}
$$

então $p_{1}-\lambda p_{0}$ é solução de (3.20) se

$$
\lambda Q_{s}=Q_{s}-\alpha_{s}<\Psi_{0}, p^{n+1}>_{h} .
$$

A solução da equação de Poisson (3.20) é única (a menos de constante). Assim, tomando-se $p^{n+1}=p_{1}-\lambda p_{0}$ e substituindo-se em (3.23), tem-se que

$$
\lambda=\frac{Q_{s}-\alpha_{s}<\Psi_{0}, p_{1}>_{h}}{Q_{s}-\alpha_{s}<\Psi_{0}, p_{0}>_{h}}
$$

O valor de $\lambda$ está bem definido pois $\left\langle\Psi_{0}, C>_{h}=\sum_{i, j}\left(\Psi_{0}\right)_{i, j} C h^{2}=0, \forall C \in \mathrm{R}\right.$ (usando equação (3.15) ). Logo, se $\bar{p}_{0}=p_{0}+C$ solução de (3.21) então $<\Psi_{0}, \bar{p}_{0}>_{h}=<\Psi_{0}, p_{0}>_{h}$. Relação similar acontece com qualquer outra solução de (3.22). Além disso, o denominador de (3.24) nunca é zero já que $\left\langle\Psi_{0}, p_{0}\right\rangle_{h}$ é negativo ou nulo. Isso decorre do fato de que

$$
<\Psi_{0}, p_{0}>_{h}=<\frac{\Delta t}{\rho Q_{s}} \mathbf{D} \cdot G p_{0}, p_{0}>_{h}=\frac{\Delta t}{\rho}<\mathbf{D} \cdot G p_{0}, p_{0}>_{h},
$$

e do fato de que o operador Laplaciano discreto $\mathbf{D} \cdot G$ é um operador negativo ou nulo. Logo $<\Psi_{0}, p_{0}>_{h} \leq 0$.

Uma vez encontrada a solução para a pressão (3.20), encontra-se a solução para o campo velocidade $\mathbf{u}^{n+1}$ usando a equação (3.18), a qual pode-se ser escrita da forma

$$
\mathbf{u}^{n+1}=\mathbf{u}^{n+1,2}-\frac{\Delta t}{\rho} G p^{n+1} .
$$

O cálculo de $p_{0}$ por (3.21) é levado a cabo só uma vez já que $\Psi_{0}$ e $Q_{s}$ são constantes no tempo, enquanto que o cálculo de $p_{1}$ por (3.22) é efetuado em cada passo do tempo. 


\subsubsection{Algoritmo do Método da Fronteira Imersa contendo uma fonte e um sumidouro}

Dado $\left(\mathbf{u}^{n}, \mathbf{X}^{n}\right)$ calcular $\left(\mathbf{u}^{n+1}, \mathbf{X}^{n+1}\right)$

Passo 1: Calcular a densidade de força elástica $\mathrm{f}^{n}$ (dependente do modelo).

Passo 2: Distribuir a densidade de força elástica aos pontos na malha do fluido por meio da equação (2.22).

Passo 3 Passo de projeção: resolver as equações de Navier-Stokes usando uma variação do Método de Projeção de Chorin, em duas etapas:

Passo 3A: Calcular um campo de velocidade auxiliar $\mathbf{u}^{n+1,2}$ usando o esquema dado pelo algoritmo de Printz [16]

$$
\begin{aligned}
\rho\left[\frac{\mathbf{u}^{n+1,0}-\mathbf{u}^{n}}{\Delta t}\right] & =\mathbf{F}^{n}, \\
\rho\left[\frac{\mathbf{u}^{n+1,1}-\mathbf{u}^{n+1,0}}{\Delta t}+u^{n} D_{x}^{0} \mathbf{u}^{n+1,1}\right] & =\mu D_{x}^{+} D_{x}^{-} \mathbf{u}^{n+1,1}, \\
\rho\left[\frac{\mathbf{u}^{n+1,2}-\mathbf{u}^{n+1,1}}{\Delta t}+v^{n} D_{y}^{0} \mathbf{u}^{n+1,2}\right] & =\mu D_{y}^{+} D_{y}^{-} \mathbf{u}^{n+1,2},
\end{aligned}
$$

onde $\mathbf{u}^{m}=\left(u^{m}, v^{m}\right)$.

Passo 3B: Decompor a velocidade auxiliar $\mathbf{u}^{n+1,2}$

$$
\begin{aligned}
\mathbf{u}^{n+1,2} & =\mathbf{u}^{n+1}+\frac{\Delta t}{\rho} G p^{n+1} \\
\text { D. } \mathbf{u}^{n+1} & =\left(Q_{s}-\alpha_{s} p_{F, S}^{n+1}\right) \Psi_{0} .
\end{aligned}
$$

As quais gerarão a equação de Poisson para a pressão

$$
\frac{\Delta t}{\rho} \mathbf{D} \cdot G p^{n+1}=\mathbf{D} \cdot \mathbf{u}^{n+1,2}-\left(Q_{s}-\alpha_{s}<\Psi_{0}, p^{n+1}>_{h}\right) \Psi_{0}
$$

Passo 4: Atualizar a posição da fronteira imersa usando (2.23). 


\subsection{Escoamentos contendo $k$ fontes e $k$ sumidouros}

$\mathrm{Na}$ seção anterior foram deduzidas equações que modelam o escoamento de um fluido viscoso contendo uma fonte e um sumidouro. O modelo pode ser geralizado para conter $k$ fontes e $k$ sumidouros. Para induzir a expressão geral, considere primeiramente a forma para a função $q(\mathrm{x}, t)$ no caso de duas fontes e dois sumidouros

$$
q(\mathrm{x}, t)=\sum_{m=1}^{2} Q_{m}(t) \Psi_{m}(\mathbf{x}),
$$

sendo

$$
\Psi_{m}(\mathbf{x})=W_{F_{m}}(\mathbf{x})-W_{S_{m}}(\mathbf{x})
$$

e

$$
Q_{m}(t)=\frac{\bar{P}_{m}-P_{m}(t)}{R_{m}}
$$

com

$$
P_{m}(t)=\int_{\Omega} p(\mathbf{x}, t)\left(W_{F_{m}}(\mathbf{x})-W_{S_{m}}(\mathbf{x})\right) d \mathbf{x}=\int_{\Omega} p(\mathbf{x}, t) \Psi_{m}(\mathbf{x}) d \mathbf{x}
$$

onde, como na seção anterior $\Psi_{m}(\mathbf{x})$ fornece a distribuição espacial para a fonte $m$ e sumidouro $m$, por meio das funções pesos $W_{F_{m}}(\mathbf{x})$ e $W_{S_{m}}(\mathbf{x})$, cada uma com suporte compacto e integral igual a um. A vazão $Q_{m}(t)$ segue o modelo de resistência linear visto anteriormente e $P_{m}(t)$ é a diferença entre a pressão média na fonte $m$ e no sumidouro $m, m=1,2$. A Figura 3.4, temos uma possível posição para as duas fontes e os dois sumidouros, assim como as vazões associadas.

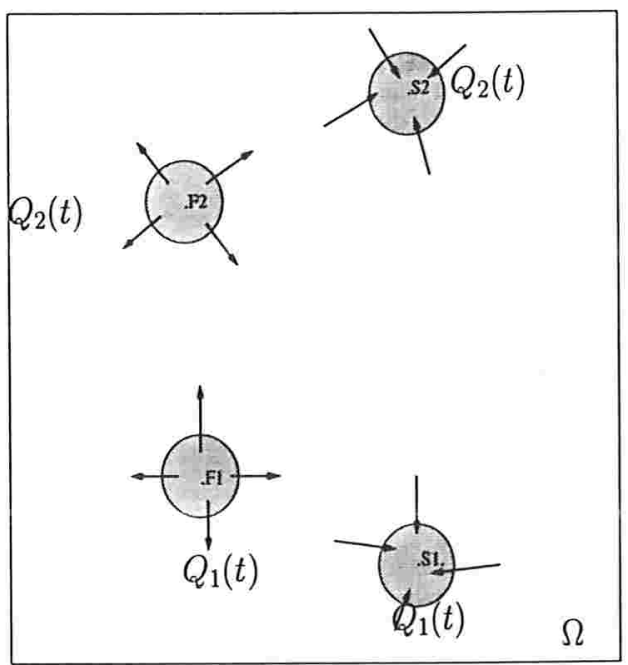

Figura 3.4: Escoamentos com duas fontes e dois sumidouros e respectivas vazões. 
O passo de decomposição (Passo 3B do algoritmo apresentado na Seção 2.3) do Método da Fronteira Imersa será modificado devido à presença das fontes e sumidouros. A forma das equações passam a ser dadas por

$$
\begin{aligned}
\rho\left(\frac{\mathbf{u}^{n+1}-\mathbf{u}^{n+1,2}}{\Delta t}\right) & =-G p^{n+1}, \\
\mathbf{D} \cdot \mathbf{u}^{n+1} & =\sum_{m=1}^{2} Q_{m}^{n+1} \Psi_{m},
\end{aligned}
$$

onde $\mathbf{u}^{n+1,2}$ é o campo de velocidade auxiliar encontrado como em (2.26). Estas equações serão resolvidas seguindo a idéia de Kayne [1], que considera o campo velocidade $\mathbf{u}^{n+1}$ e o campo pressão $p^{n+1}$ como uma superposição de soluções, isto é,

$$
\begin{aligned}
& \mathbf{u}^{n+1}=\overline{\mathbf{u}}^{n+1}+\sum_{m=1}^{2} \mathbf{b}_{m} Q_{m}^{n+1} \\
& p^{n+1}=\bar{p}^{n+1}+\sum_{m=1}^{2} r_{m} Q_{m}^{n+1}
\end{aligned}
$$

onde o par $\left(\overline{\mathbf{u}}^{n+1}, \bar{p}^{n+1}\right)$ são soluções das equações:

$$
\begin{aligned}
\rho\left(\frac{\overline{\mathbf{u}}^{n+1}-\mathbf{u}^{n+1,2}}{\Delta t}\right) & =-G \bar{p}^{n+1}, \\
\mathbf{D} \cdot \overline{\mathbf{u}}^{n+1} & =0,
\end{aligned}
$$

e $\mathrm{b}_{m}, r_{m}$ são funções definidas na malha e que devem ser encontradas. As equações da forma (3.36) e (3.37) geram a equação de Poisson para o campo escalar $\bar{p}^{n+1}$, neste caso dada por

$$
\frac{\Delta t}{\rho} \mathbf{D} \cdot G \bar{p}^{n+1}=\mathbf{D} \cdot \mathbf{u}^{n+1,2}
$$

Substituindo-se (3.34) e (3.35) em (3.32)

$$
\rho\left(\frac{\overline{\mathbf{u}}^{n+1}+\sum_{m=1}^{2} \mathbf{b}_{m} Q_{m}^{n+1}-\mathbf{u}^{n+1,2}}{\Delta t}\right)=-G \bar{p}^{n+1}-\sum_{m=1}^{2} G r_{m} Q_{m}^{n+1}
$$

e usando-se (3.36), tem-se

$$
\sum_{m=1}^{2}\left(\frac{\rho \mathbf{b}_{m}}{\Delta t}+G r_{m}\right) Q_{m}^{n+1}=0
$$

A equação anterior deve ser independente da vazão $Q_{m}^{n+1}$. Isto significa que o termo dentro do parênteses deve ser nulo para se ter verificada a equação. Assim,

$$
\rho \frac{\mathbf{b}_{m}}{\Delta t}=-G r_{m}
$$


De forma similar, substituindo-se (3.34) em (3.33), obtém-se

$$
\mathbf{D} \cdot \overline{\mathbf{u}}^{n+1}+\sum_{m=1}^{2} \mathbf{D} \cdot \mathrm{b}_{m} Q_{m}^{n+1}=\sum_{m=1}^{2} Q_{m}^{n+1} \Psi_{m},
$$

e usando-se (3.37), resulta

$$
\sum_{m=1}^{2}\left(\mathbf{D} \cdot \mathbf{b}_{m}-\Psi_{m}\right) Q_{m}^{n+1}=0
$$

Pelo mesmo argumento da não dependência da vazão, a expressão dentro do parênteses acima deve ser nula, isto é,

$$
\mathbf{D} \cdot \mathbf{b}_{m}=\Psi_{m} .
$$

As equações (3.38) e (3.39) geram a equação de Poisson para o campo escalar $r_{m}$

$$
\frac{\Delta t}{\rho} \mathbf{D} \cdot G r_{m}=-\Psi_{m}
$$

que após resolvida equação fornece o campo vetorial $\mathbf{b}_{m}$ por intermédio de (3.38). Note que $\Psi_{m}$, o segundo membro de (3.39), é conhecida, sendo a distribuição espacial adotada para a $m$-ésima fonte e para o $m$-ésimo sumidouro. Condições do tipo periódica são empregadas. A diferença entre as equações de Poisson associadas ao par $\left(\bar{p}^{n+1}, \overline{\mathbf{u}}^{n+1}\right)$ e $\left(r_{m}, \mathbf{b}_{m}\right), m=1,2$, está no fato de que estas últimas são calculadas só uma vez, pois as funções $r_{m}$ e $\mathbf{b}_{m}, m=1,2$, não dependen do tempo.

Das equações (3.30) e (3.31) tem-se

$$
P_{m}(t)=\int p(\mathbf{x}, t) \Psi_{m}(\mathbf{x}) d \mathbf{x}=\overline{P_{m}}-R_{m} Q_{m}(t) .
$$

A substituição da expressão para a pressão (3.35) na forma discretizada da expressão acima resulta em

$$
\sum_{l, s}\left(\bar{p}^{n+1}+\sum_{i=1}^{2} r_{i} Q_{i}^{n+1}\right) \Psi_{m} h^{2}=\bar{P}_{m}-R_{m} Q_{m}^{n+1}
$$

ou ainda

$$
\begin{gathered}
\sum_{l, s} \bar{p}^{n+1} \Psi_{m} h^{2}+\sum_{i=1}^{2}\left(\sum_{\Omega_{h}} r_{i} \Psi_{m} h^{2}\right) Q_{i}^{n+1}=\bar{P}_{m}-R_{m} Q_{m}^{n+1}, \\
<\bar{p}^{n+1}, \Psi_{m}>_{h}+\sum_{i=1}^{2}<r_{i}(\mathrm{x}), \Psi_{m}(\mathrm{x})>_{h} Q_{i}^{n+1}=\bar{P}_{m}-R_{m} Q_{m}^{n+1},
\end{gathered}
$$

onde $m=1,2$, e $<r_{i}, \Psi_{m}>_{h} \approx<r_{i}, \Psi_{m}>=\int_{\Omega} r_{i}(\mathbf{x}) \Psi(\mathbf{x}) d \mathbf{x}$, a qual pode ser escrita para $m=1$ e $m=2$ como

$$
\begin{aligned}
& \sum_{i=1}^{2}<r_{i}, \Psi_{1}>_{h} Q_{i}^{n+1}+R_{1} Q_{1}^{n+1}=\bar{P}_{1}-<\bar{p}^{n+1}, \Psi_{1}>_{h}, \\
& \sum_{i=1}^{2}<r_{i}, \Psi_{2}>_{h} Q_{i}^{n+1}+R_{2} Q_{2}^{n+1}=\bar{P}_{2}-<\bar{p}^{n+1}, \Psi_{2}>_{h} .
\end{aligned}
$$


Estas equações podem ser escritas na forma matricial como $\mathbf{A ~}^{n+1}=\mathbf{B}^{n+1}$, onde

$$
\begin{gathered}
\mathbf{A}=\left(\begin{array}{cc}
<r_{1}, \Psi_{1}>_{h}+R_{1} & <r_{2}, \Psi_{1}>_{h} \\
<r_{1}, \Psi_{2}>_{h} & <r_{2}, \Psi_{2}>_{h}+R_{2}
\end{array}\right) \\
\mathbf{B}^{n+1}=\left(\begin{array}{c}
\bar{P}_{1}-<\bar{p}^{n+1}, \Psi_{1}>_{h} \\
\bar{P}_{2}-<\bar{p}^{n+1}, \Psi_{2}>_{h}
\end{array}\right) \text { e } \mathrm{Q}^{n+1}=\left(\begin{array}{c}
Q_{1}^{n+1} \\
Q_{2}^{n+1}
\end{array}\right)
\end{gathered}
$$

Em cada passo do tempo, a matriz A é constante pois $r_{i}, \Psi_{j}$ e $R_{k}$ são constantes, enquanto o vetor $\mathbf{B}^{n+1}$ se modifica.

Este procedimento para o caso de duas fontes e dois sumidouros pode ser geralizado. No que segue, faz-se um breve resumo dos modelos adotados para os elementos que são empregados na simulação de fontes e de sumidouros, assim como sua respectiva generalização!

(a) cada fonte $m$ tem a ela associada um sumidouro $m$ de mesma intensidade. O modelo para a vazão na fonte e no sumidouro são dados pela a Lei de Poiseuille, (3.10), a qual é um modelo de resistência linear. A vazão da fonte $m$ é igual a do sumidouro $m$. Assim a conservação de massa do fluido é preservada no domínio;

(b) a função que fornece a distribuição espacial para a fonte e sumidouro $m$ é dada pela função $\Psi_{m}(\mathbf{x})=W_{F_{m}}(\mathbf{x})-W_{S_{m}}(\mathbf{x})$, onde as funções peso $W_{F_{m}}(\mathbf{x})$ e $W_{S_{m}}$ têm suportes compactos e integrais iguais a um;

(c) as formas adotadas para o campo vetorial velocidade e escalar de pressão, no passo de decomposição do algoritmo do Método da Fronteira Imersa, apresentadas na Seção 2.3, são supostas como sendo a superposição de soluções, isto é,

$$
\begin{aligned}
& \mathbf{u}(\mathbf{x}, t)=\overline{\mathbf{u}}(\mathbf{x}, t)+\sum_{m=1}^{k} \mathbf{b}_{m}(\mathbf{x}) Q_{m}(t) \\
& p(\mathbf{x}, t)=\bar{p}(\mathbf{x}, t)+\sum_{m=1}^{k} r_{m}(\mathbf{x}) Q_{m}(t)
\end{aligned}
$$

onde $k$ indica o número de fontes e sumidouros. Foram introduzidas $k$ campos vetoriais, $\mathbf{b}_{m}$, e $k$ campos escalares $r_{m}, m=1, \ldots, k$. Ao usar as equações do passo de decomposição, encontra-se uma equação de Poisson associada a cada par de fonte e sumidouro $\left(\mathbf{b}_{m}, r_{m}\right)$, assim como uma para o par $\left(\overline{\mathbf{u}}^{n+1}, \bar{p}^{n+1}\right)$. O cálculo da vazão, leva a um sistema linear de equações de ordem $k \times k, \mathbf{A ~ Q ~}^{n+1}=\mathbf{B}^{n+1}$, onde 


$$
\begin{aligned}
& \mathbf{A}=\left(\begin{array}{cccccc}
\bar{a}_{1,1}+R_{1} & \bar{a}_{1,2} & \bar{a}_{1,3} & \cdots & & \bar{a}_{1, k} \\
\bar{a}_{2,1} & \bar{a}_{2,2}+R_{2} & \bar{a}_{2,3} & & & \vdots \\
\vdots & & \ddots & & & \\
& & & & & \\
\vdots & & & & & \\
\bar{a}_{1, k} & \ldots & & & \bar{a}_{k-1, k} & \bar{a}_{k, k}+R_{k}
\end{array}\right) \\
& \mathbf{B}^{n+1}=\left(\begin{array}{c}
\bar{P}_{1}-<\bar{p}^{n+1}, \Psi_{1}>_{h} \\
\bar{P}_{2}-<\bar{p}^{n+1}, \Psi_{2}>_{h} \\
\vdots \\
\bar{P}_{k}-<\bar{p}^{n+1}, \Psi_{k}>_{h}
\end{array}\right) \text { e } \mathrm{Q}^{n+1}=\left(\begin{array}{c}
Q_{1}^{n+1} \\
Q_{2}^{n+1} \\
\vdots \\
\\
Q_{k}^{n+1}
\end{array}\right)
\end{aligned}
$$

$\operatorname{com} \bar{a}_{i, j}=<r_{j}, \Psi_{i}>_{h}, i, j=1, \ldots, k$.

Para finalizar, observe que o caso para o qual se tem $k_{1}$ fontes (sumidouros) e $k_{2}$ sumidouros (fontes), onde $k_{1} \neq k_{2}$, pode também ser abordado pela metodologia exposta, bastando para isto tomar apropriadamente fontes (ou sumidouros) coincidentes. Na Figura 3.5, temos um gráfico que ilustra o caso de $k_{1}=2, k_{2}=1$.

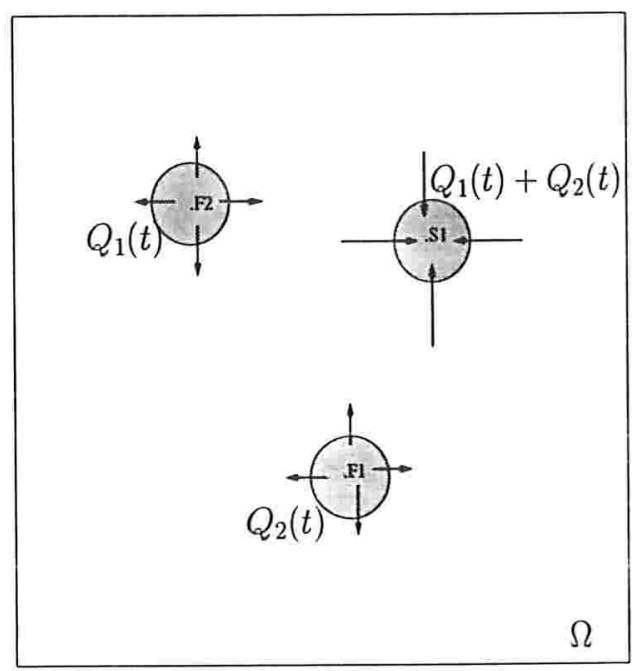

Figura 3.5: Escoamentos com duas fontes e um sumidouro e respectivas vazões. 
CAPÍtulo 4

\section{Testes numéricos}

Neste capítulo, serão apresentados os testes utilizados para validar a implementação computacional do Método da Fronteira Imersa, incluindo os testes que incorporam os modelos adotados para fontes e sumidouros.

$\mathrm{Na}$ Seção 4.1, apresentam-se as normas que serão empregadas assim como as relações entre os erros absolutos para aproximações de uma ordem dada.

Na Seção 4.2, verifica-se a ordem do passo de projeção (Seção 2.3, Passo 3) que é o responsável por resolver as Equações de Navier-Stokes no método computacional da fronteira imersa.

Na Seção 4.3, realizam-se testes com o objetivo de comprovar a efetividade do modelo de resistência linear adotado para a vazão na fonte e no sumidouro.

$\mathrm{Na}$ Seção 4.4, é apresentado a simulação numérica do escoamento laminar entre placas paralelas e infinitas.

\subsection{Preliminares: normas e análise de convergência}

O domínio $\Omega=[0,1] \times[0,1]$ é discretizado empregando-se uma malha computacional uniforme $\Omega_{h}=\left\{(i h, j h) \mid 0 \leq i, j \leq N, h=\frac{1}{N}\right\}$, onde $h$ indica o espaçamento dos pontos nas duas direções $x, y$. A malha pode ser decomposta em dois subconjuntos, $\Omega_{h}^{o}$ e $\partial \Omega_{h}$. O primeiro denota o conjunto de pontos interiores de $\Omega_{h}$ e o segundo os pontos fronteira.

Sejam $f, g: \Omega_{h} \subseteq \mathbf{R}^{2} \rightarrow \mathbf{R}$ e $\mathbf{v}, \mathbf{w}: \Omega_{h} \subseteq \mathbf{R}^{2} \rightarrow \mathbf{R}^{2}$ funções escalares e vetoriais, respectivamente, definidas na malha $\Omega_{h}$. Denotando-se por $f_{z}=f(i h, j h)$ e por $\mathbf{v}_{z}=\left(v_{1_{z}}, v_{2_{z}}\right)=$ $\left(v_{1}(i h, j h), v_{2}(i h, j h)\right)$, onde $z=[i, j], 0 \leq i, j \leq N$, os produtos internos para funções escalares e vetoriais podem ser definidos respectivamente como 


$$
\begin{aligned}
<f, g>_{h} & =\sum_{z \in \Omega_{h}^{o}} f_{z} g_{z} h^{2}+\frac{1}{2} \sum_{z \in \partial \Omega_{h}} f_{z} g_{z} h^{2} \\
& =\sum_{i=0, j=0}^{N-1} f_{z} g_{z} h^{2} \quad \text { (condição de contorno periódica) } \\
<\mathbf{v}, \mathbf{w}>_{h} & =\sum_{i=1}^{2}<v_{i}, w_{i}>_{h} .
\end{aligned}
$$

Serão empregadas na análise de convergência, a seguir, as normas $L_{2}$ induzidas pelos produtos internos (4.1) e (4.2),

$$
\begin{aligned}
\|f\|_{2} & =\left(<f, f>_{h}\right)^{1 / 2} \\
\|\mathbf{v}\|_{2} & =\left(<\mathbf{v}, \mathbf{v}>_{h}\right)^{1 / 2}
\end{aligned}
$$

e as normas infinito

$$
\begin{aligned}
\|f\|_{\infty} & =\max _{z \in \Omega_{h}}\left|f_{z}\right| \\
\|\mathbf{v}\|_{\infty} & =\max _{i}\left\|v_{i}\right\|_{\infty}, \quad i=1,2 .
\end{aligned}
$$

Para analisar numericamente a convergência do método, será suposto que a solução fornecida por ele tem expansão assintótica nas potências do espaçamento da malha. Nas malhas uniformes empregadas, por exemplo, a função escalar $\phi$ tem solução numérica $\phi(x, t, h)$ a qual, por hipótese, pode ser escrita na forma assintótica

$$
\phi(\mathbf{x}, t, h)=\phi_{e}(\mathbf{x}, t)+\mathbf{E}_{q}(\mathbf{x}, t) h^{q}+\mathbf{E}_{q+1}(\mathbf{x}, t) h^{q+1}+\ldots
$$

para $h$ suficientemente pequeno, onde $\phi_{e}(\mathrm{x}, t)$ é a solução exata e os coeficientes $\mathbf{E}_{q}(\mathbf{x}, t), i=$ $q, q+1, \ldots$, independem de $h$.

Se a solução exata é conhecida, após ter sido encontrada a solução aproximada $\phi(x, t, h)$ para algum $h$, calcula-se para o mesmo ponto $(\mathbf{x}, t)$ a aproximação $\phi(\mathbf{x}, t, h / 2)$. Assim obtém-se, em primeira aproximação,

$$
\begin{gathered}
\phi(\mathbf{x}, t, h) \approx \phi_{e}(\mathbf{x}, t)+\mathbf{E}_{q}(\mathbf{x}, t) h^{q}, \\
\phi(\mathrm{x}, t, h / 2) \approx \phi_{e}(\mathrm{x}, t)+\mathbf{E}_{q}(\mathrm{x}, t) \frac{h^{q}}{2^{q}} .
\end{gathered}
$$

Das duas aproximações anteriores, os erros entre a solução exata e as aproximadas para malhas de espaçamentos $h$ e $h / 2$, satisfazem a razão

$$
\frac{\left\|\phi(\mathbf{x}, t, h)-\phi_{e}(\mathbf{x}, t)\right\|}{\left\|\phi(\mathbf{x}, t, h / 2)-\phi_{e}(\mathbf{x}, t)\right\|} \approx 2^{q},
$$


onde a norma utilizada pode ser (4.3) ou (4.5).

- Seguindo este raciocínio, para aproximações de funções vetoriais que tem uma expansão assintótica, obtém-se

$$
\frac{\left\|\mathbf{v}(\mathbf{x}, t, h)-\mathbf{v}_{e}(\mathbf{x}, t)\right\|}{\left\|\mathbf{v}(\mathbf{x}, t, h / 2)-\mathbf{v}_{e}(\mathbf{x}, t)\right\|} \approx 2^{q}
$$

onde $\mathbf{v}_{e}(\mathbf{x}, t)$ é a solução exata e, mais uma vez, $\|\cdot\|$ representa qualquer uma das normas, $\|\cdot\|_{2}$ ou $\|.\|_{\infty}$, definidas em (4.4) e (4.6), respectivamente.

Fazendo-se uso de (4.7) (ou (4.8)), tem-se que a razão entre os erros, na malha de espaçamento $h$ e $h / 2$, para métodos de ordem $q=1,2$ e 3, devem ser aproximadamente 2,4 e 8 , respectivamente.

\subsection{Resolução numérica das equações de Navier-Stokes}

O passo de projeção (Seção 2.3, Passo 3), responsável por resolver numericamente as equações de Navier-Stokes no Método da Fronteira Imersa, emprega uma aproximação de segunda ordem no espaço e de primeira ordem no tempo.

Para examinar a resolução numérica das equações de Navier-Stokes separadamente, um problema modelo "suave" é utilizado, evitando-se termos forçantes singulares como o dado em (2.10). Nenhuma fronteira imersa será definida e o termo forçante $\mathbf{F}$ provirá de forças exteriores.

Para verificar numericamente a ordem de convergência do passo de projeção, foram consideradas as funções periódicas no domínio $\Omega=[0,1] \times[0,1]$ para o campo de velocidade e de pressão (Lai [10]):

$$
\begin{aligned}
& \mathbf{u}_{e}(\mathbf{x}, t)=\mathbf{a}_{1} \cdot \sin \left(\mathbf{b}_{1} \cdot \mathbf{x}+w_{1} \cdot t\right)+\mathbf{a}_{2} \cdot \sin \left(\mathbf{b}_{2} \cdot \mathbf{x}+w_{2} \cdot t\right) \\
& p_{e}(\mathbf{x}, t)=c_{1} \cdot \sin \left(\mathbf{b}_{1} \cdot \mathbf{x}+w_{1} \cdot t\right)+c_{2} \cdot \sin \left(\mathbf{b}_{2} \cdot \mathbf{x}+w_{2} \cdot t\right)
\end{aligned}
$$

onde

$$
\begin{aligned}
& \mathbf{a}_{1}=(-1,2), \quad \mathbf{a}_{2}=(2,-2), \\
& \mathbf{b}_{1}=2 \pi(2,1), \quad \mathbf{b}_{2}=2 \pi(1,1), \\
& c_{1}=c_{2}=1, \quad w_{1}=w_{2}=w=0.1 .
\end{aligned}
$$

As expressões para o campo densidade de força, $\mathbf{F}=\left(F_{x}, F_{y}\right)$, são dadas por

$$
\begin{aligned}
F_{x}= & \rho[-w(\cos A-2 \cos B)+4 \pi(\sin (A-B)]+2 \pi(2 \cos A+\cos B) \\
& -4 \mu \pi^{2}(5 \sin A-4 \sin B) \\
F_{y}= & \rho[2 \pi(\cos A-\cos B)+4 \pi(2 \sin B \cos A-\sin A \cos B)]+2 \pi(\cos A+\cos B) \\
& -4 \mu \pi^{2}(4 \sin B-10 \sin A),
\end{aligned}
$$


com

$$
A=4 \pi x+2 \pi y+w t \quad \text { e } \quad B=2 \pi x+2 \pi y+w t .
$$

A condição inicial para o campo de velocidade é dada por $\mathbf{u}_{e}(\mathbf{x}, 0.0)$ e os valores dos parâmetros $\rho$ e $\mu$ foram escolhidos como sendo $1.0 \mathrm{~g} / \mathrm{cm}^{3}$ e $0.0001 \mathrm{~g} / \mathrm{cm} . \mathrm{s}$, respectivamente.

As soluções aproximadas obtidas através do passo de projeção exibirão uma taxa de convergência de segunda ordem quando $\Delta t=O\left(h^{2}\right)$. Na Tabela 4.1, mostram-se os erros entre a solução exata e a aproximada, para o instante de tempo $t_{f}=0.3125$, em malhas de tamanho $N \times N, N=16,32,64,128$, e $\Delta t=h^{2} / 2$. Além disso, são apresentadas as razões entre os erros nas malhas de tamanho $N \times N$ e $2 N \times 2 N$. Estes erros foram calculados tanto na norma $L_{2}$, como na norma $L_{\infty}$ para funções vetoriais. Comparando-se a razões encontradas nesta tabela com a relação (4.8), observa-se que o método de projeção é de segunda ordem, a ordem esperada teoricamente.

\begin{tabular}{|c|c|c|c|c|c|c|c|}
\hline \hline$h$ & $1 / 16$ & & $1 / 32$ & & $1 / 64$ & & $1 / 128$ \\
\hline$N$ & 16 & razão & 32 & razão & 64 & razão & 128 \\
\hline \hline$\left\|\mathbf{u}_{N}-\mathbf{u}_{e}\right\|_{2}$ & 1.250248 & 3.412003 & 0.366427 & 3.883699 & 0.094350 & 3.978998 & 0.023712 \\
$\left\|\mathbf{u}_{N}-\mathbf{u}_{e}\right\|_{\infty}$ & 2.422854 & 3.402732 & 0.712032 & 3.8641429 & 0.184266 & 3.9735622 & 0.046373 \\
\hline \hline
\end{tabular}

Tabela 4.1: Passo de projeção: erros cometidos, no campo de velocidade, e razão entre eles no tempo $t=0.3125 s$.

\subsection{Introdução de uma fonte e um sumidouro no modelo}

O objetivo aqui é testar o desempenho do método na presença de uma fonte e um sumidouro. Como foi descrito na Seção 3.2, neste caso, a equação de continuidade pode ser reescrita como

$$
\nabla \cdot \mathbf{u}(\mathbf{x}, t)=q(\mathbf{x}, t)
$$

onde $q(\mathbf{x}, t)=Q(t) \Psi_{0}(\mathbf{x})$.

O modelo adotado para a vazão é o modelo de resistência linear, dado por

$$
Q(t)=Q_{s}-\alpha_{s} P_{F, S}(t),
$$

onde $Q_{s}$ e $\alpha_{s}$ são constantes a serem selecionadas e a função $\Psi_{0}(\mathbf{x})$ é dada por

$$
\Psi_{0}(\mathbf{x})=\left\{\begin{array}{rr}
W_{F}\left(\mathbf{x}-\mathbf{P}_{F}\right), & \forall \mathbf{x} \in V_{\mathbf{P}_{F}} ; \\
-W_{S}\left(\mathbf{x}-\mathbf{P}_{S}\right), & \forall \mathbf{x} \in V_{\mathbf{P}_{S}} ; \\
0, & \text { caso contrário }
\end{array}\right.
$$


$\mathrm{O}$ centro da fonte está localizado no ponto $\mathbf{P}_{F}$ e do sumidouro no ponto $\mathbf{P}_{S}$, e os respectivos suportes compactos, $V_{\mathbf{P}_{F}}$ e $V_{\mathbf{P}_{S}}$ serão tomadas como sendo os quadrados de aresta com comprimento $4 h_{0}$, neles centrados.

As funções adotadas para $W_{F}(\mathbf{x})$ e $W_{S}(\mathbf{x})$ devem ser positivas com integrais iguais a um. Neste caso, serão tomadas $W_{F}(\mathbf{x})=W_{S}(\mathbf{x})=\delta_{h_{o}}(\mathbf{x})$, onde esta última é a aproximação da função delta de Dirac bidimensional dada por (2.21) com $h_{o}=\frac{1}{32}$, a qual será mantida fixa qualquer que seja a malha computacional considerada. $\mathrm{O}$ centro da fonte foi tomado como $\mathbf{P}_{F}=(0.25,0.5)$; do sumidouro, $\mathbf{P}_{S}=(0.75,0.5)$. A Figura 4.1 mostra o gráfico da função $\Psi_{0}$ (constante no tempo) numa malha de $64 \times 64$.

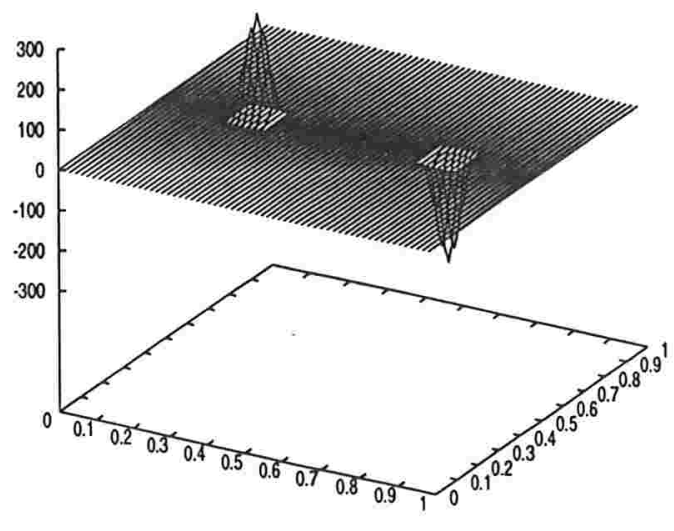

Figura 4.1: Gráfico da $\Psi_{0}$ numa malha $64 \times 64$.

Para não violar a condição de incompressibilidade no domínio de integração considerado, $\Omega=[0,1] \times[0,1]$, é necessário que a quantidade de fluido injetada pela fonte seja a mesma que é absorvida pelo sumidouro. Empregando-se uma fonte e um sumidouro de mesma intensidade, este fato pode ser verificado experimentalmente. No instante inicial $(\mathrm{t}=0.0)$, marca-se um conjunto de partículas do fluido sobre duas circunferências, uma centrada em $\mathbf{P}_{F}$ e outra sobre $\mathbf{P}_{S}$ (ver Figura 4.2). Pela ação da fonte, espera-se que o primeiro conjunto de partículas se expanda, enquanto que o segundo se contraia pela remoção de fluido no sumidouro.

Devido ao fato de que a fonte e o sumidouro possuem a mesma vazão, a variação absoluta de área em cada conjunto deve ser a mesma e deve ter sinais opostos. 


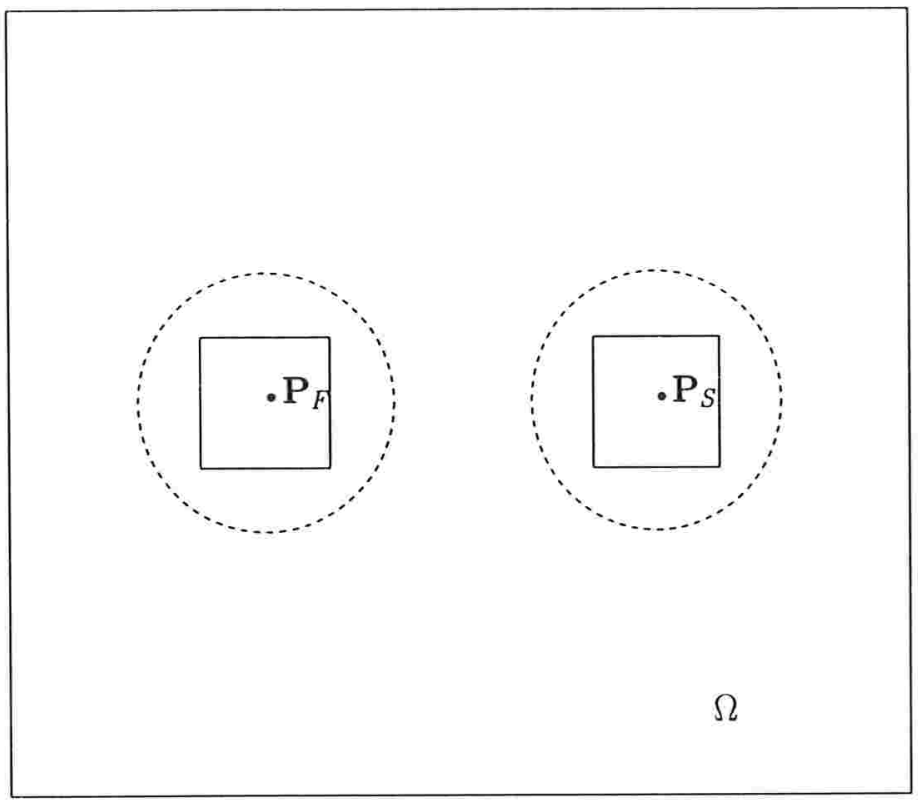

Figura 4.2: Localização inicial das circunferências: uma ao redor da fonte e outra ao redor do sumidouro.

Uma relação importante existente entre a variação de área e a vazão, que será de utilidade na validação da implementação computacional, será deduzida a seguir. Para isso, a região que tem como fronteira o primeiro conjunto de pontos (que varia com o tempo) será denotada por $A_{t}$ e à medida de sua área por $A(t)$. A variação da massa no tempo da região $W_{t}=A_{t} \times\left[0, L_{z}\right]$ (problema tridimensional associado ao bidimensional), onde $L_{z}>0$ é o comprimento na direção do eixo $z$ considerada, é dada por

$$
\frac{d m(t)}{d t}=\frac{d}{d t} \int_{W_{t}} \rho d x d y d z
$$

onde $m(t)$ e $\rho$ são a massa e a densidade naquela região. Usando-se o Teorema do Transporte (vide Apêndice C) e sendo $\rho$ constante, obtém-se

$$
\begin{aligned}
\frac{d m(t)}{d t} & =\int_{W_{t}}\left(\frac{\partial \rho}{\partial t}+\nabla \cdot(\rho \mathbf{u})\right)(\mathbf{x}, t) d x d y d z \\
& =\int_{W_{t}} \rho \nabla \cdot \mathbf{u}(\mathbf{x}, t) d x d y d z \\
& =\rho \int_{W_{t}} Q(t) \Psi_{0}(\mathbf{x}) d x d y d z \\
& =\rho Q(t) \int_{W_{t}} W_{F}\left(\mathbf{x}-\mathbf{P}_{F}\right) d x d y d z \\
& =\rho Q(t) \int_{[0, L z]}\left(\int_{A_{t}} W_{F}\left(\mathbf{x}-\mathbf{P}_{F}\right) d x d y\right) d z \\
& =\rho Q(t) L_{z},
\end{aligned}
$$


como $m(t)=\rho A(t) L_{z}$, então

$$
\frac{d A(t)}{d t}=Q(t)
$$

A igualdade acima revela a taxa teórica de variação para a área da região que contém a fonte.

Considerando-se $\rho=1.0 \mathrm{~g} / \mathrm{cm}^{3}, \mu=0.01 \mathrm{~g} / \mathrm{cm} . \mathrm{s}$, e o fluido em repouso para $t=0.0 \mathrm{~s}$, o esquema numérico foi integrado até $t=1.2 \mathrm{~s}$ com os valores $Q_{s}=0.01 \mathrm{~cm}^{2} / \mathrm{s}$ e $\alpha_{s}=10^{-7} \mathrm{~cm}^{3} \mathrm{~s} / \mathrm{g}$.

Para t suficientemente grande, neste problema, a taxa de variação teórica da área é dada por $\frac{d A(t)}{d t}=Q^{*}=Q_{s}-\alpha_{s} P_{F, S}^{*}$, onde $Q^{*}$ e $P_{F, S}^{*}$ são a vazão e a diferença entre as pressões na fonte e no sumidouro no regime permanente. Devido à escolha dos parâmetros, tem-se nesse caso $\alpha_{s} P_{F, S}^{*}<<Q_{s}$, o que justifica a utilização de $\frac{d A(t)}{d t}=Q_{s}$ como aproximação da taxa teórica de variação de área. A Figura 4.3, $(a)$ - $(d)$, mostra o gráfico da diferença entre uma aproximação da taxa teórica e a taxa obtida numericamente da variação de área da região marcada ao redor da fonte. Para cada instante de tempo tem-se

$$
e\left(t_{k}\right)=\left|\frac{d A\left(t_{k}\right)}{d t}-\frac{A^{k}-A^{k-1}}{t_{k}-t_{k-1}}\right|
$$

onde $A^{k}$ é a área da circunferência ao redor da fonte no instante de tempo $t_{k}$, calculada numericamente mediante a rotina que foi implementada de acordo com a fórmula apresentada no Apêndice D.

Quando comparadas entre si as taxas de variação da área verificou-se uma diferença de 5.04\% na malha $16 \times 16$ sendo a variação ainda menor para as malhas com $N=32,64$ e 128 .

A Figura 4.4, $(a)-(d)$, mostra a razão entre as variações obtidas numericamente das áreas das circunferências ao redor da fonte e ao redor do sumidouro. Verificou-se que, no pior caso a razão obtida foi de $90.7 \%$ na malha de $16 \times 16$, contra uma razão de $99.92 \%$ na malha $128 \times 128$.

Nas Figuras 4.5 e 4.6 mostram-se as simulações para as malhas de tamanho $32 \times 32$ e $64 \times 64$, respectivamente. Em qualquer das malhas os conjuntos de pontos foram trasladados usando-se a equação (2.23). 


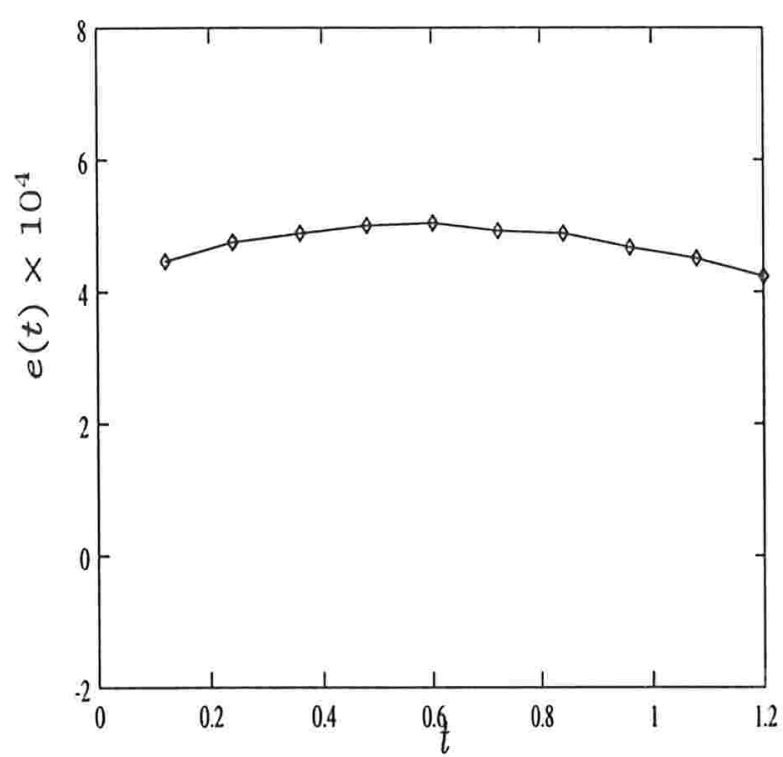

(a)

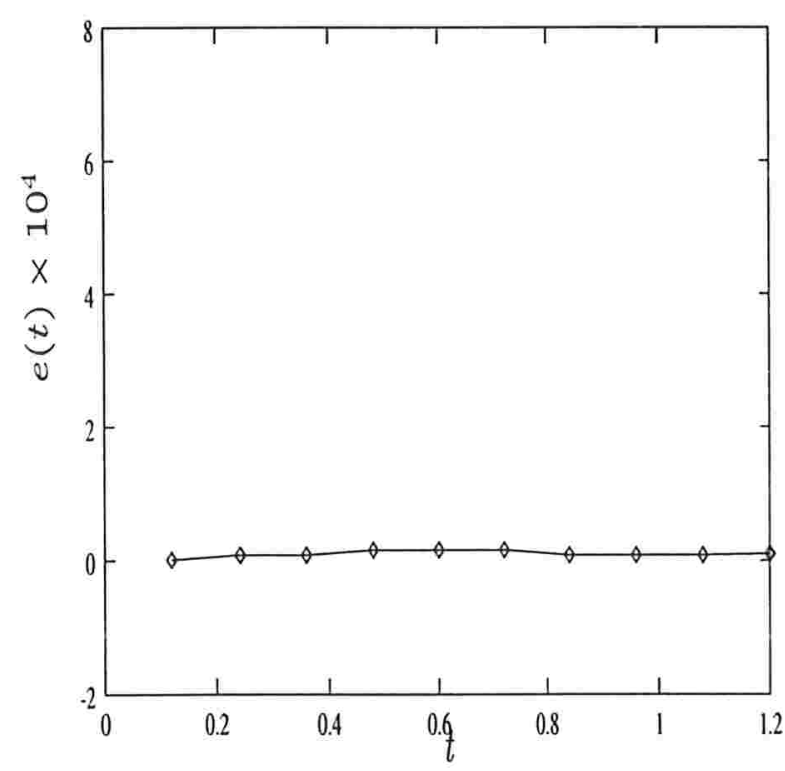

(c)

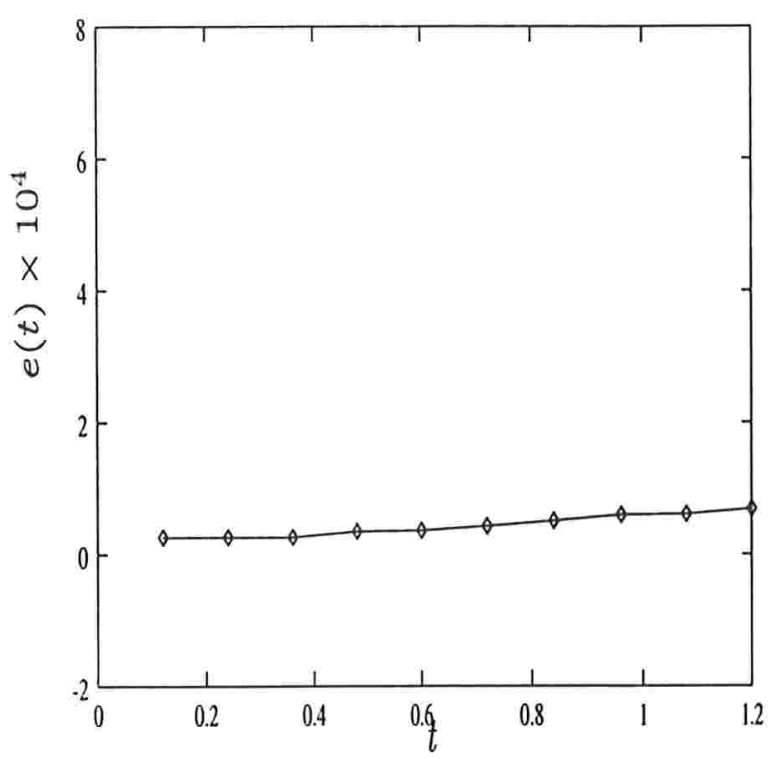

(b)

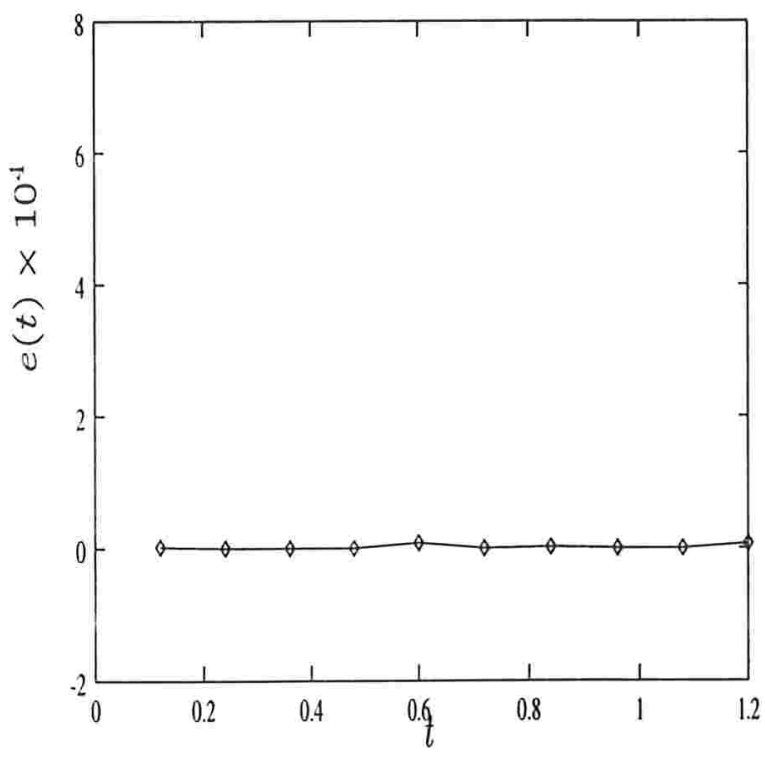

(d)

Figura 4.3: Medida da diferença entre a taxa de variação da área teórica e experimentalmente na simulação do escoamento de um fluido contendo uma fonte e um sumidouro; $Q_{s}=0.01 \mathrm{e}$ $\alpha_{s}=10^{-7}$ 

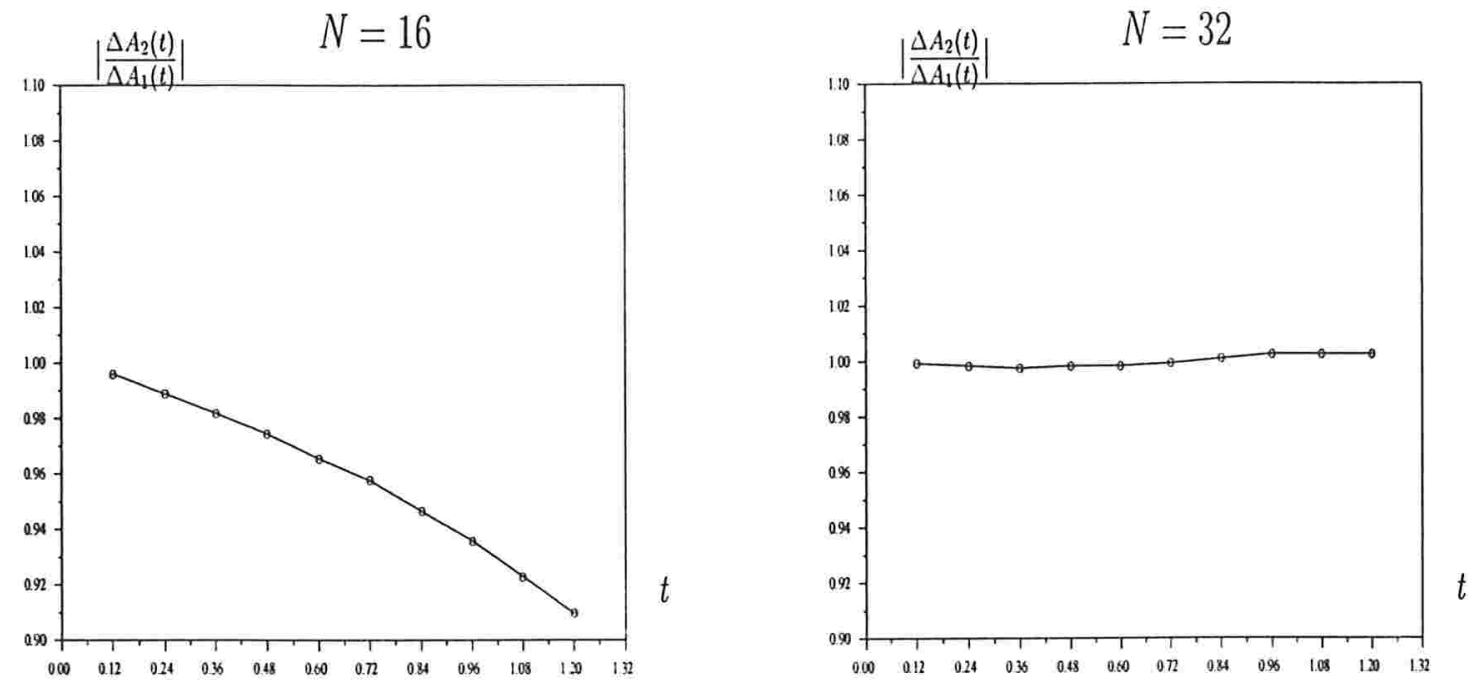

(a)

(b)
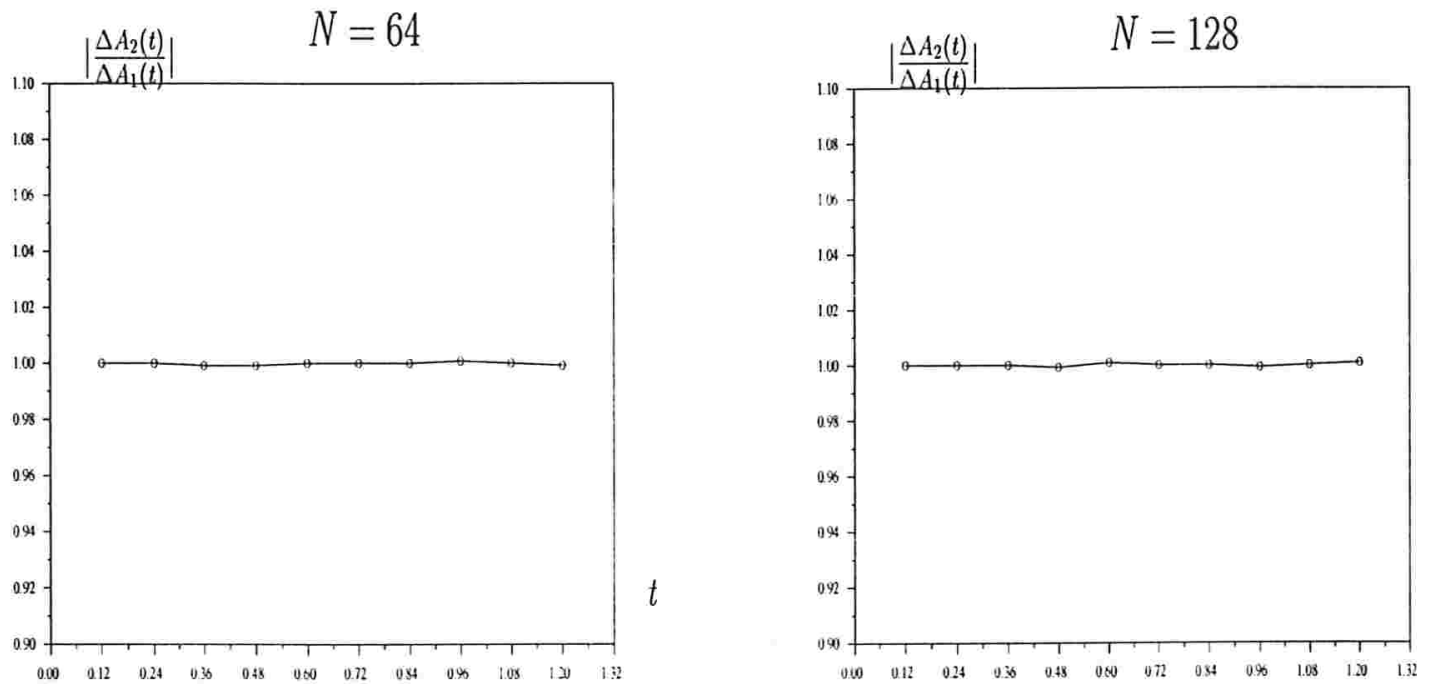

(c)

(d)

Figura 4.4: Razão entre as variações experimentais das áreas das circunferências ao redor da fonte e ao redor do sumidouro; $Q_{s}=0.01$ e $\alpha_{s}=10^{-\vec{t}}$. 


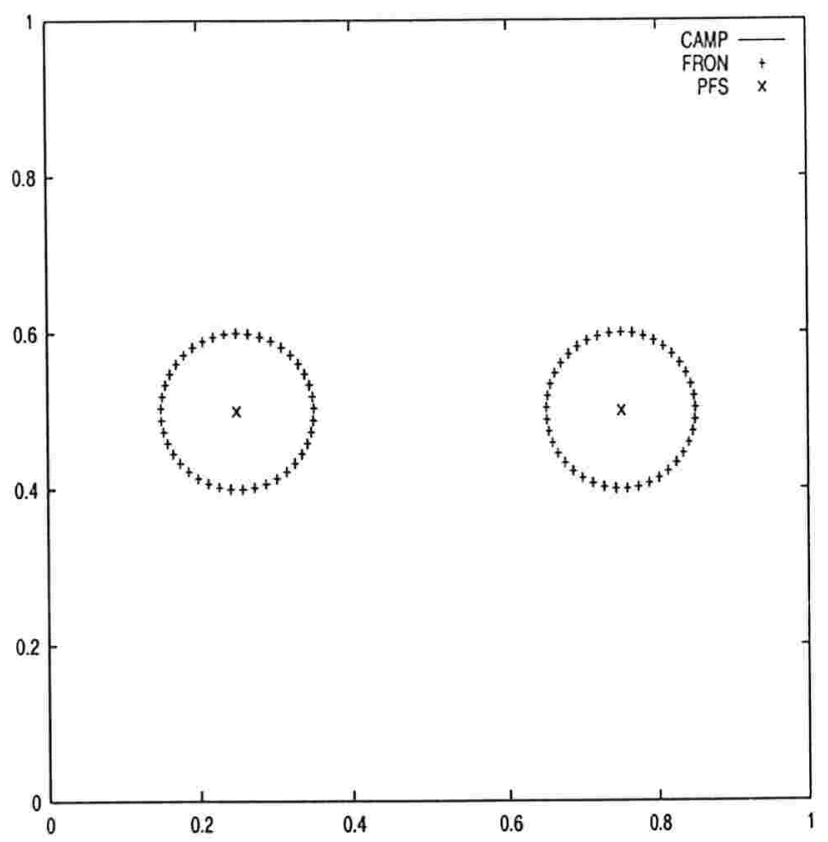

(a) $t=0.000000$

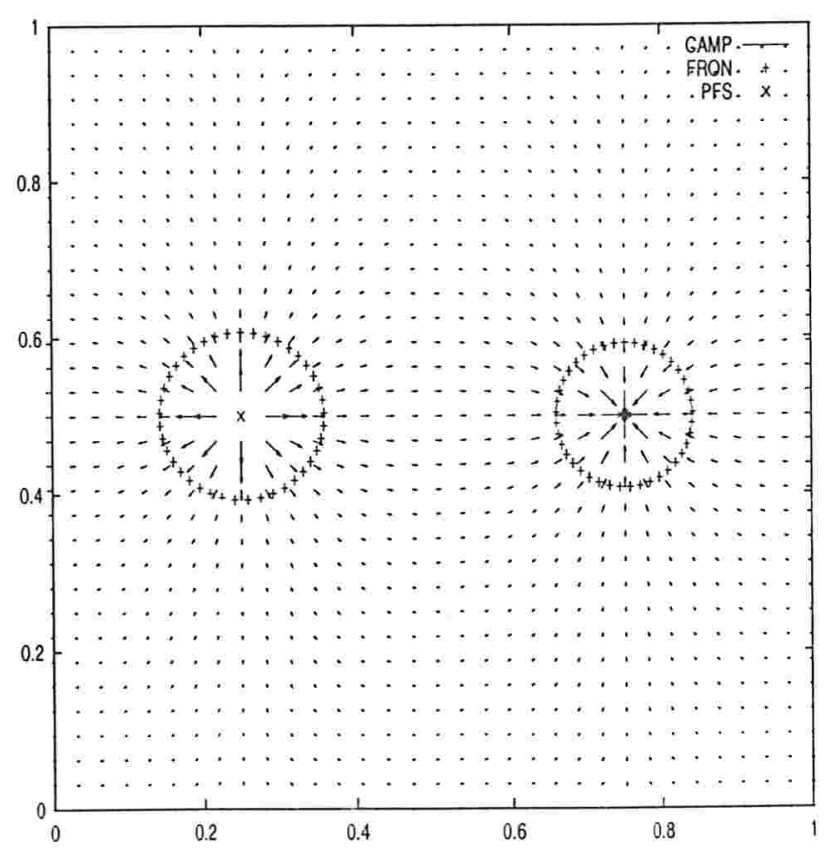

(b) $t=0.480103$ 


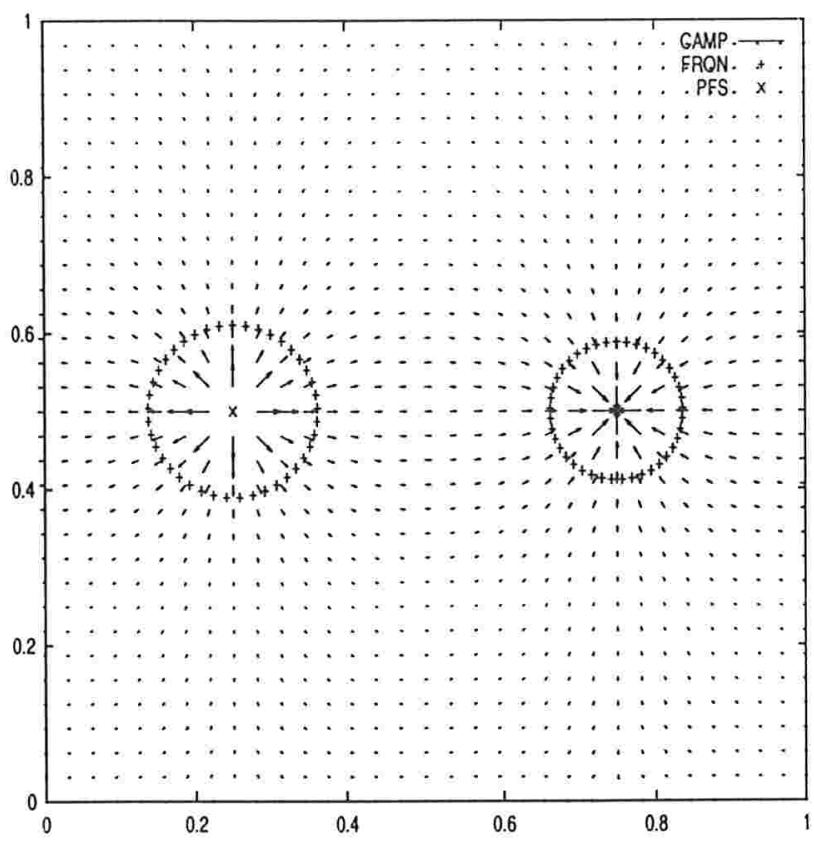

(c) $t=0.720093$

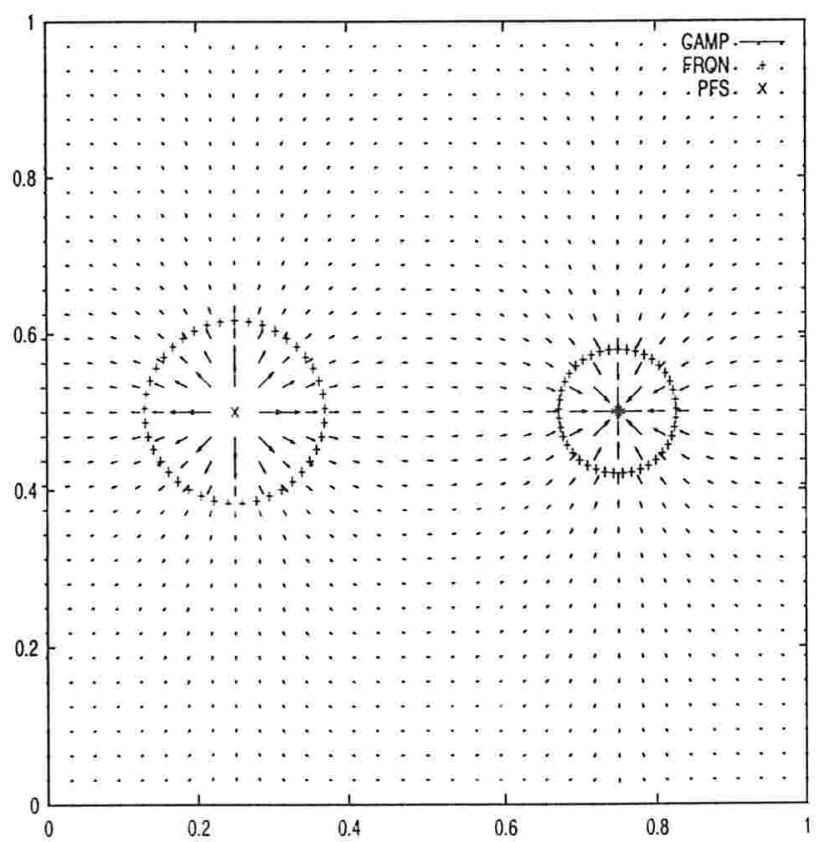

(d) $t=1.200000$

Figura 4.5: Simulação do escoamento de um fluido contendo uma fonte e um sumidouro; malha $32 \times 32 ; Q_{s}=0.01$ e $\alpha_{s}=1 e-7, t=1.2 s$. 


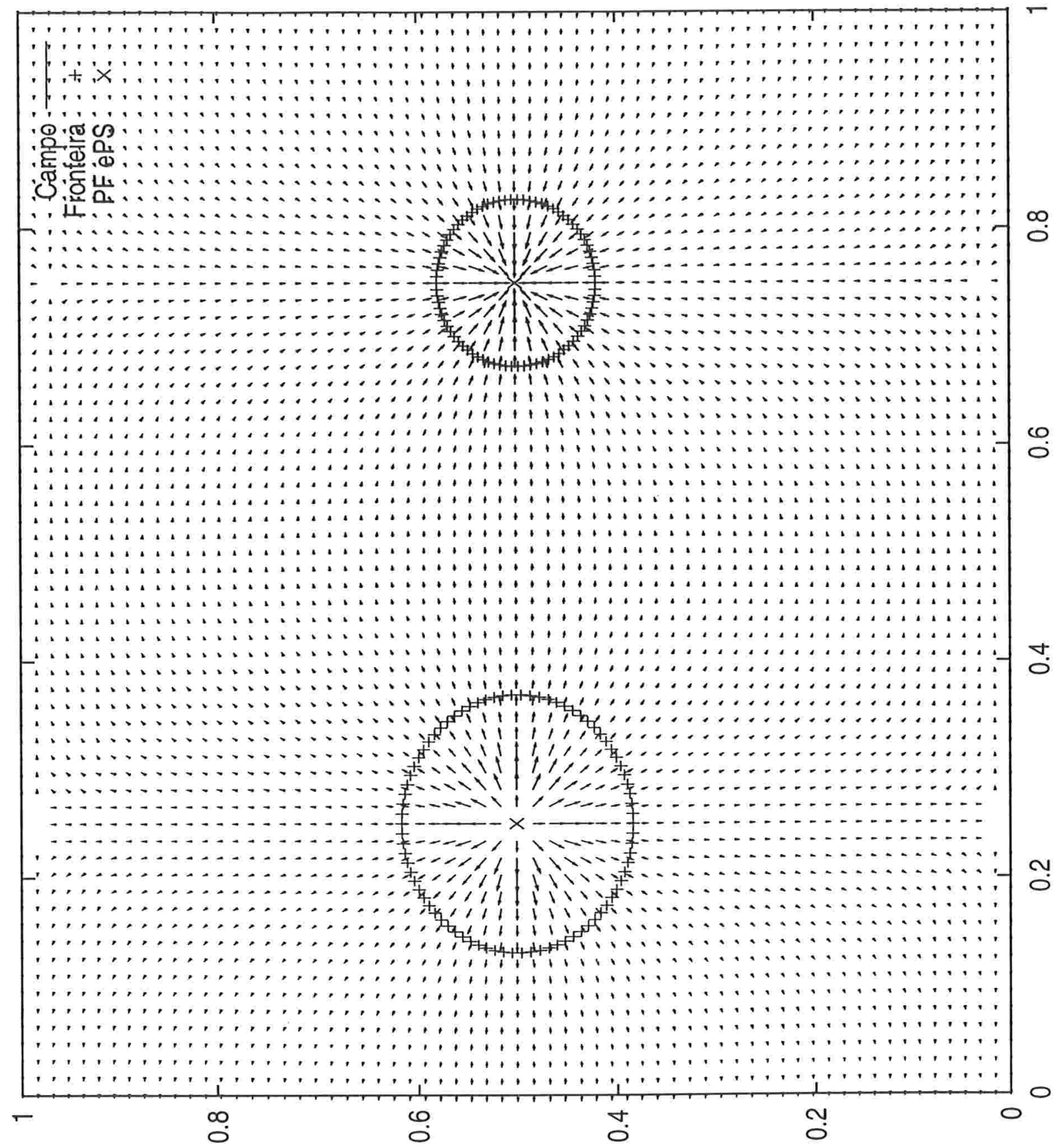

Figura 4.6: Simulação do escoamento de um fluido contendo uma fonte e um sumidouro; malha $64 \times 64 ; Q_{s}=0.01$ e $\alpha_{s}=1 e-7$ e $t=1.2 s$. 


\subsection{Escoamento laminar entre placas paralelas}

\subsubsection{Modelo bidimensional}

O modelo bidimensional adotado para simular o escoamento laminar entre duas placas paralelas é ilustrado na Figura 4.7. O domínio $\Omega$ considerado é o plano de corte $x y$ ( Figura 4.7-(a)).
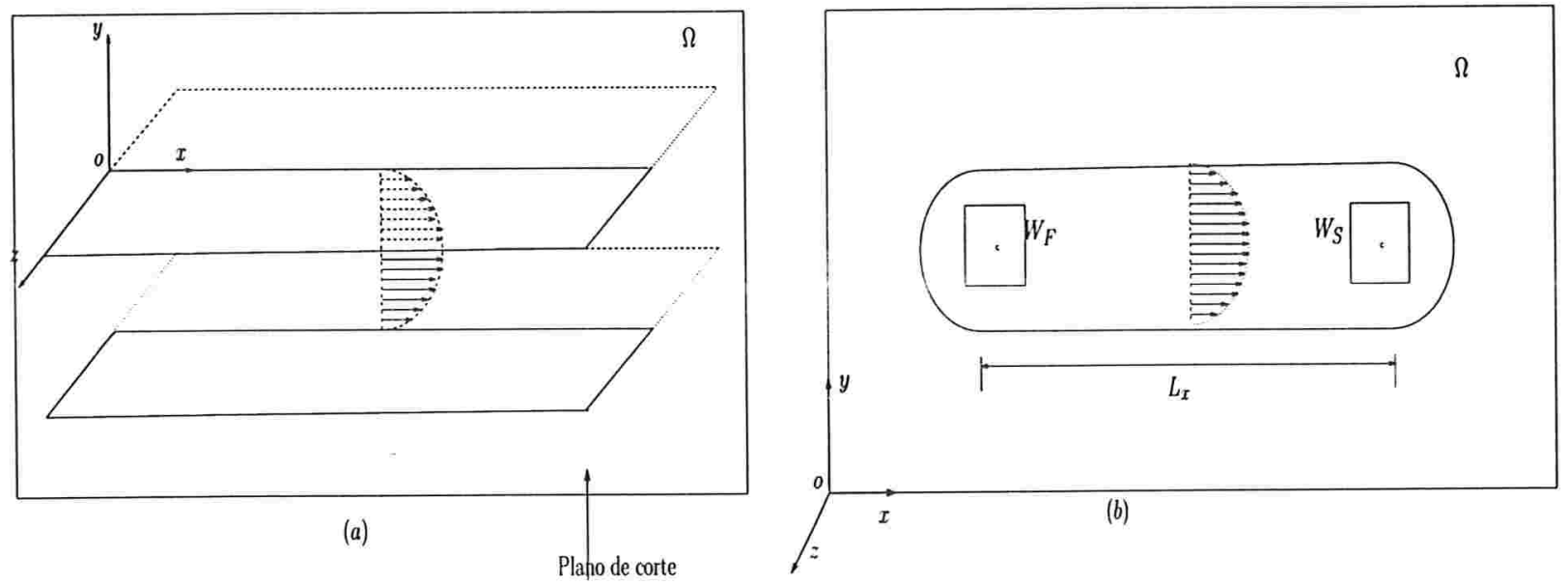

Figura 4.7: Modelo bidimensional para simular o escoamento entre placas paralelas.

Para se representar este problema no domínio finito de integração $\Omega$, utilizou-se como artifício ção de "tampas" nas "extremidades" das placas para evitar perda de vazão fornecida ula fonte e, para modelar o gradiente de pressão responsável por mover este fluido, uma fonte e um sumidouro são posicionados em $W_{F}$ e $W_{S}$ (Figura 4.7-(b)).

Adotando-se mais uma vez o modelo linear para a vazão (4.11), em regime permanente, a "vazão" bidimensional no problema será dada por $Q^{*}=Q_{s}-\alpha_{s} P_{F, S}^{*}$. Integrando-se na direção do eixo $z$ de 0 a $L_{z}$ tem-se como resultado a vazão propriamente dita, $L_{z} Q^{*}$.

A massa injetada pela fonte, $\rho L_{z} Q^{*}$, deve ser a mesma que passa pelo centro (conservação da massa). Assim, empregando-se (3.6) tem-se

$$
\rho L_{z} Q^{*}=-\rho \frac{2 L_{z} C}{3 \mu} a^{3} .
$$

Logo,

$$
Q *=-\frac{2 C}{3 \mu} a^{3},
$$

e,

$$
-C=\frac{3 \mu Q^{*}}{2 a^{3}} .
$$




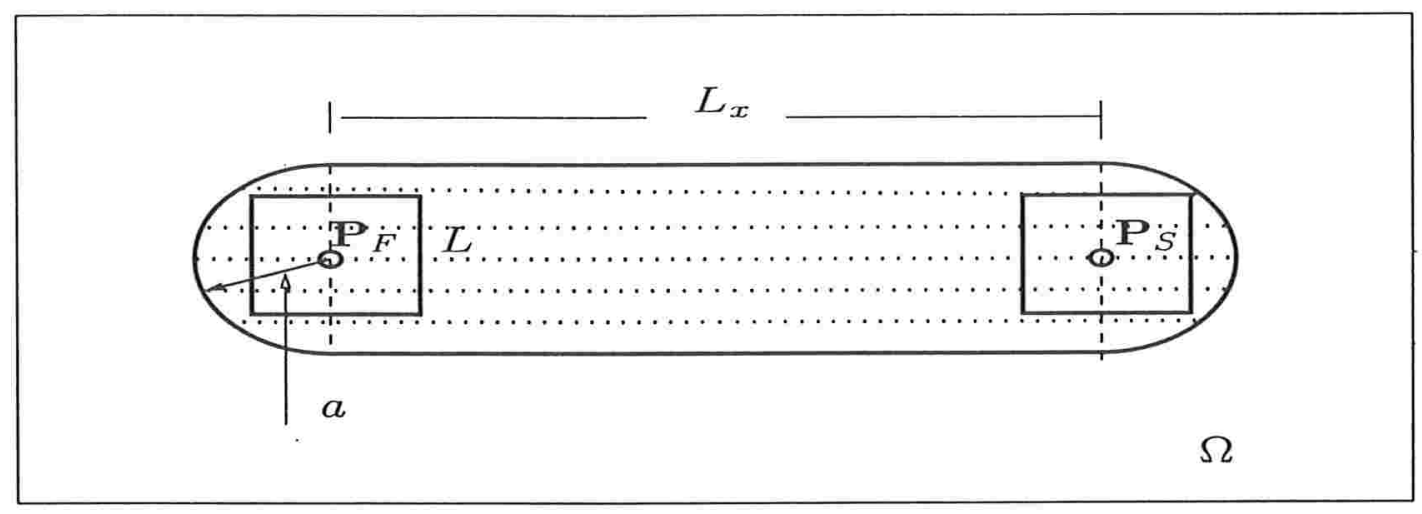

Figura 4.8: Secçao transversal do modelo tridimensional adotado para o escoamento laminar dentro das placas; configuração inicial (tempo $=0.0$ ). Os quadrados de lado $L=4 h_{0}$, representam o domínio da fonte e do sumidouro com centros em $\mathbf{P}_{F}$ e $\mathbf{P}_{S}$, respectivamente.

Substituindo-se o valor acima em (3.4), obtém-se

$$
u(y)=\frac{3 Q^{*}}{4 a^{3}}\left(a^{2}-y^{2}\right)=\frac{3\left(Q_{s}-\alpha_{s} P_{F, S}^{*}\right)}{4 a^{3}}\left(a^{2}-y^{2}\right)
$$

como solução exata da velocidade dentro do canal em função da vazão injetada pela fonte bidimensional, no regime permanente.

\subsubsection{Condições iniciais na simulação}

A fronteira está inicialmente configurada como é mostrado na Figura 4.8. É considerada como tendo propriedades elásticas com massa desprezível, a qual está imersa dentro de um fluido viscoso e incompressível.

Para a densidade de força elástica, $\mathbf{f}(s, t)$, adotou-se um tipo de "força restauradora", a qual obedece a Lei de Hooke

$$
\mathbf{f}(s, t)=-K\left(\mathbf{X}(s, t)-\mathbf{X}_{0}(s)\right)
$$

onde $\mathbf{X}_{0}(s)$ é a posição de equilíbrio que desejamos que a fronteira mantenha e, $K$ uma constante de proporcionalidade. Este tipo de força faz com que o ponto que está fora da posição de equilíbrio retorne para a posição anterior.

As medidas adotadas para o modelo da seção transversal do canal bidimensional foram: comprimento lateral $L_{x}=0.582 \mathrm{~cm}$ e $a=0.11 \mathrm{~cm}$. Os modelos adotados para a distribuição espacial da fonte e do sumidouro são as mesmas que os usados na seção 4.3. O valor do lado do quadrado do domínio da fonte e do sumidouro foram iguais a $L=4 h_{0}=0.1455 \mathrm{~cm}$, o centro delas são $\mathbf{P}_{F}=(0.25,0.5)$ e $\mathbf{P}_{S}=(0.75,0.5)$, respectivamente. 
O fluido foi inicialmente considerado em repouso, $\mathrm{u}(\mathrm{x}, 0)=0$, e os parâmetros adotados para a densidade e viscosidade do fluido foram $\rho=1.0 \mathrm{~g} / \mathrm{cm}^{3}$ e $\mu=0.01 \mathrm{~g} / \mathrm{cm} . \mathrm{s}$. Os parâmetros para a vazão, $Q(t)$, foram $Q_{s}=0.01 \mathrm{~cm}^{2} / \mathrm{s}$ e $\alpha_{s}=0.00001 \mathrm{~cm}^{3} \mathrm{~s} / \mathrm{g} . \mathrm{s}$. O valor da constante de proporcionalidade $K=10^{6} \mathrm{dyn} / \mathrm{cm}$. O valor adotado para esta constante tem como intenção manter a característica física das placas paralelas, isto é deseja-se modelar sua rigidez. Dependendo do valor que seja empregado para esta constante, pode-se modelar fronteiras mais flexíveis.

\subsubsection{Resultados}

Devido à escolha dos parâmetros de simulação, o perfil de velocidade previsto teoricamente no interior do canal em regime permanente, (4.13), pode ser aproximado por

$$
u(y)=\frac{3 Q_{s}}{4 a^{3}}\left(a^{2}-y^{2}\right),
$$

pois, novamente, esta escolha de parâmetros faz com que $\alpha_{s} P_{F, S}^{*}<<Q_{s}$. Considerou-se que foi alcanzado o regime permanente no tempo $t=1.0 \mathrm{~s}$, devido ao pequeno cambio observado nos valores da vazão (observe-se a Figura 4.9). Pode-se ver que, conforme se aumenta o tamanho da malha, o valor da vazão aproxima-se a $Q_{s}=0.01$.

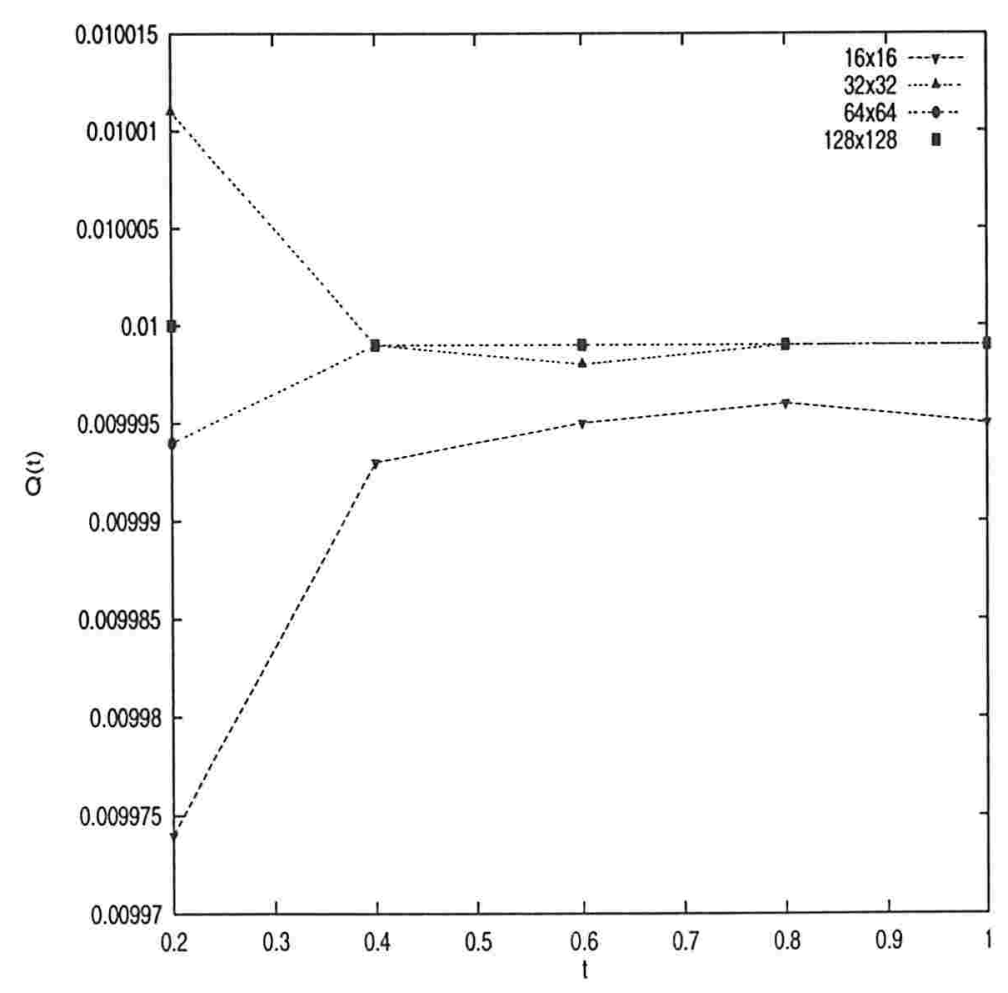

Figura 4.9: Valores da vazão $Q(t)$. 
A análise de convergência foi feita comparando-se a velocidade no meio do canal dada por (4.14) e obtida numericamente no instante de tempo $t=1.0 \mathrm{~s}$, quando o escoamento já se encontra em regime permanente (número de Reynolds $\mathrm{R}=\frac{\rho L_{x}\left\|\mathbf{u}_{128}\right\|_{\infty}}{\mu}=3.9681924$ )

Na Tabela 4.2, estão os erros obtidos para as malhas de tamanho $N \times N$, onde $N=16,32,64$ e 128.

\begin{tabular}{|c|c|c|c|c|c|c|c|}
\hline \hline$h$ & $1 / 16$ & & $1 / 32$ & & $1 / 64$ & & $1 / 128$ \\
\hline$N$ & 16 & razão & 32 & razão & 64 & razão & 128 \\
\hline \hline$\left\|u_{x_{N}}-u_{x_{e}}\right\|_{2}$ & 0.024751 & 3.678805 & 0.006728 & 2.2419194 & 0.003001 & 2.1841339 & 0.001374 \\
$\left\|u_{x_{N}}-u_{x_{e}}\right\|_{\infty}$ & 0.026520 & 2.7194422 & 0.009752 & 2.2854464 & 0.004267 & 2.1983514 & 0.001941 \\
\hline \hline
\end{tabular}

Tabela 4.2: Escoamento laminar entre placas paralelas: erros cometidos, no campo de velocidade no meio do canal, e razão entre eles no tempo $t=1.0 \mathrm{~s}$.

Nota-se pelos resultados obtidos que o método apresenta um comportamento como o esperado para um método de primeira ordem. Este fato, concorda com diversas observações experimentais feita por outros autores [17], [10], [19] e [3]. Na Tabela 4.3, mostra-se os respectivos erros relativos encontrados na tabela anterior, para as malhas e tamanho $N \times N, N=16,32,64$ e 128, sendo menor comforme a malha se torna mas fina.

Na Figura 4.10, comparam-se os perfis de velocidade obtidos numericamente para as malhas te tamanho $N \times N, N=16,32,64$ e 128, com o perfil da velocidade prevista teoricamente, ¿imado por (4.14). É possível observar que, conforme a malha se torna mais fina, o perfil ue velocidade obtido numericamente aproxima-se do perfil esperado, o parabólico.

\begin{tabular}{|c|c|c|c|c|}
\hline \hline$N$ & 16 & 32 & 64 & 128 \\
\hline \hline$\frac{\left\|u_{x_{N}}-u_{x_{e}}\right\|_{2}}{\left\|u_{x_{e}}\right\|_{2}}$ & 0.40 & 0.11 & 0.04 & 0.02 \\
\hline$\frac{\left\|u_{x_{N}}-u_{x_{e}}\right\|_{\infty}}{\left\|u_{x_{e}}\right\|_{\infty}}$ & 0.38 & 0.14 & 0.06 & 0.028 \\
\hline \hline
\end{tabular}

Tabela 4.3: Erros relativos no tempo $t=1.0 \mathrm{~s}$.

Na Figura 4.11, mostra-se o escoamento dentro do canal no instante de tempo $t=1.0 \mathrm{~s}$, para malhas de tamanho $16 \times 16$ e $32 \times 32$, enquanto que nas Figuras 4.12 e 4.13 , este escoamento é mostrado nas mesmas condições para malhas de tamanho $64 \times 64$ e $128 \times 128$, respectivamente. 


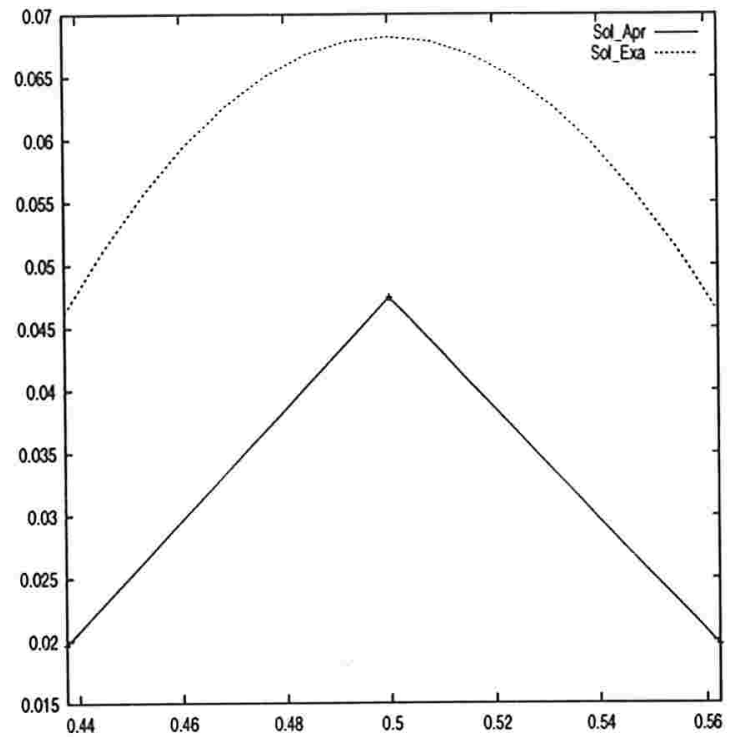

(a) $N=16$

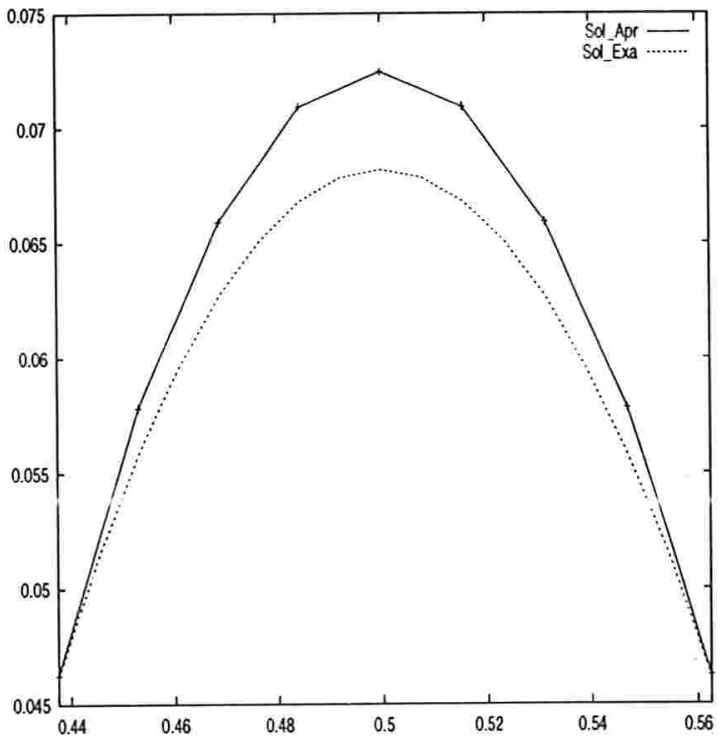

(c) $N=64$

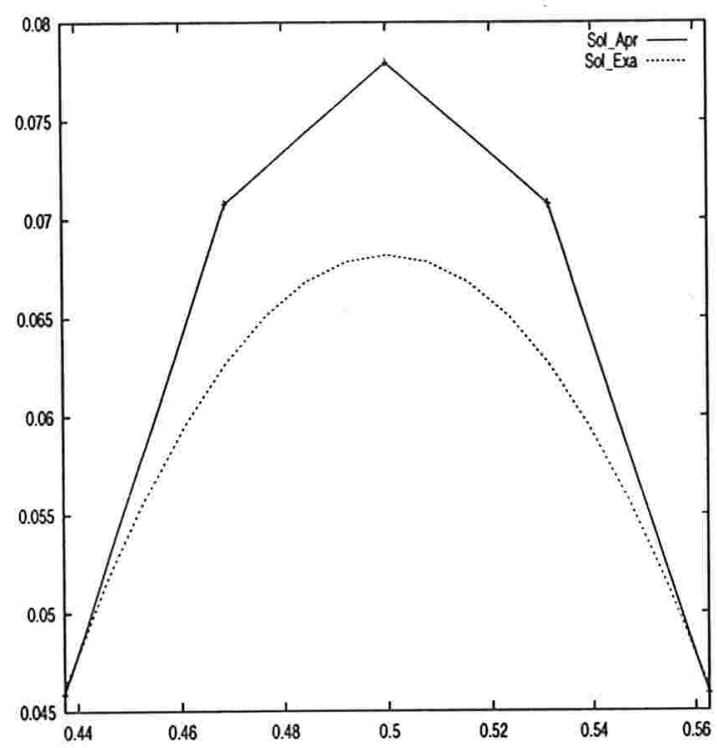

(b) $N=32$

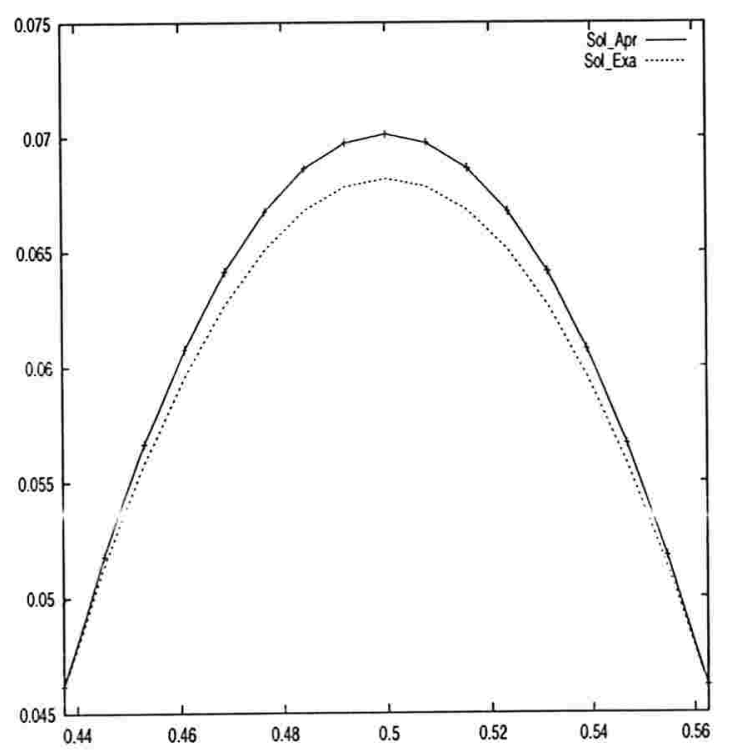

(d) $N=128$

Figura 4.10: Perfil de velocidades na metade do canal. 


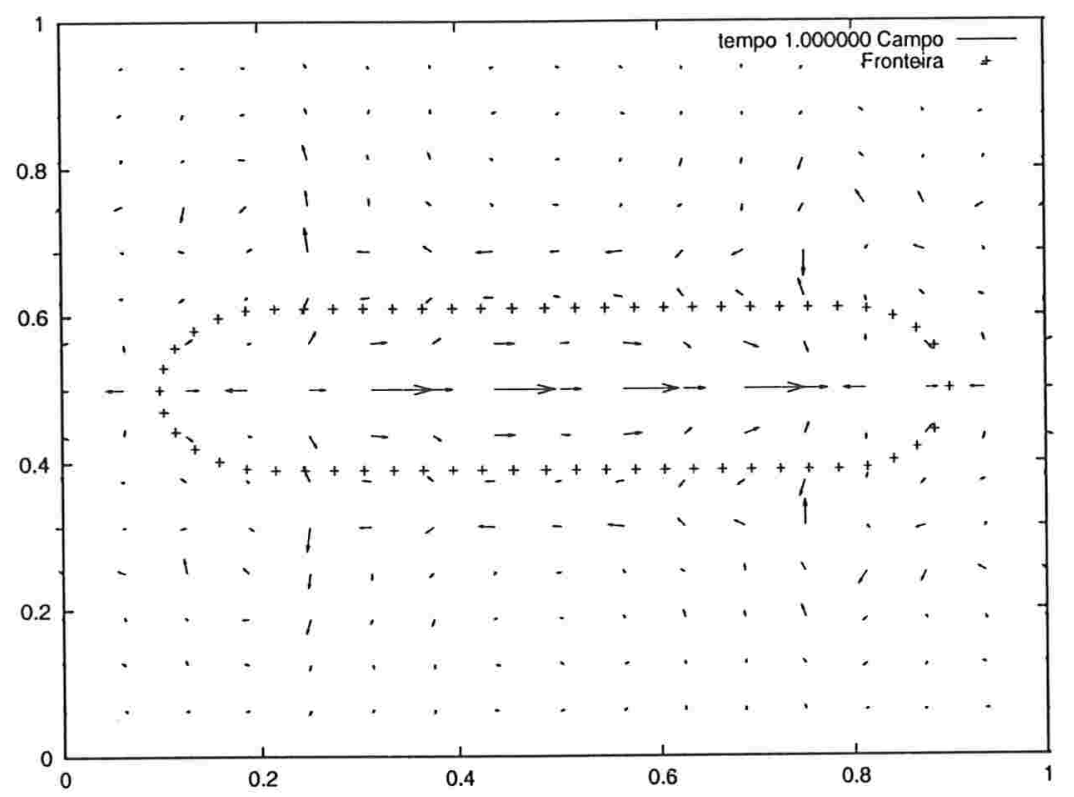

(a) $N=16$

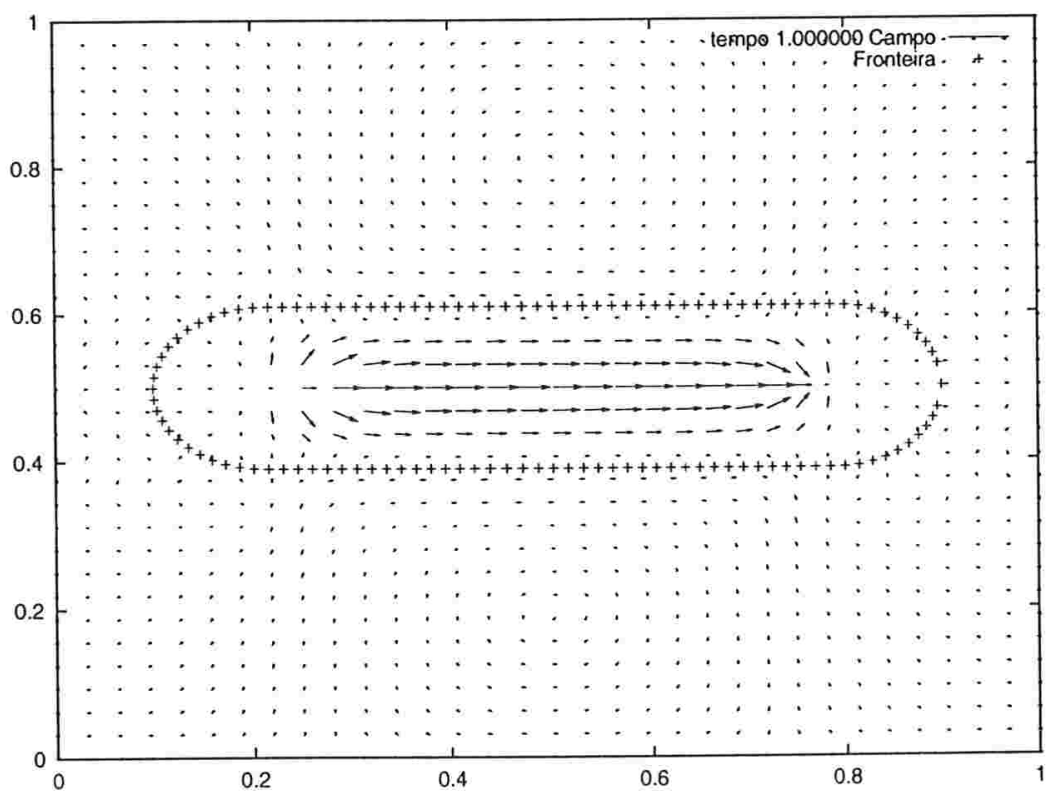

(b) $N=32$

Figura 4.11: Quadro da simulação no tempo $t=1.0$; malha $16 \times 16$ e $32 \times 32$. 


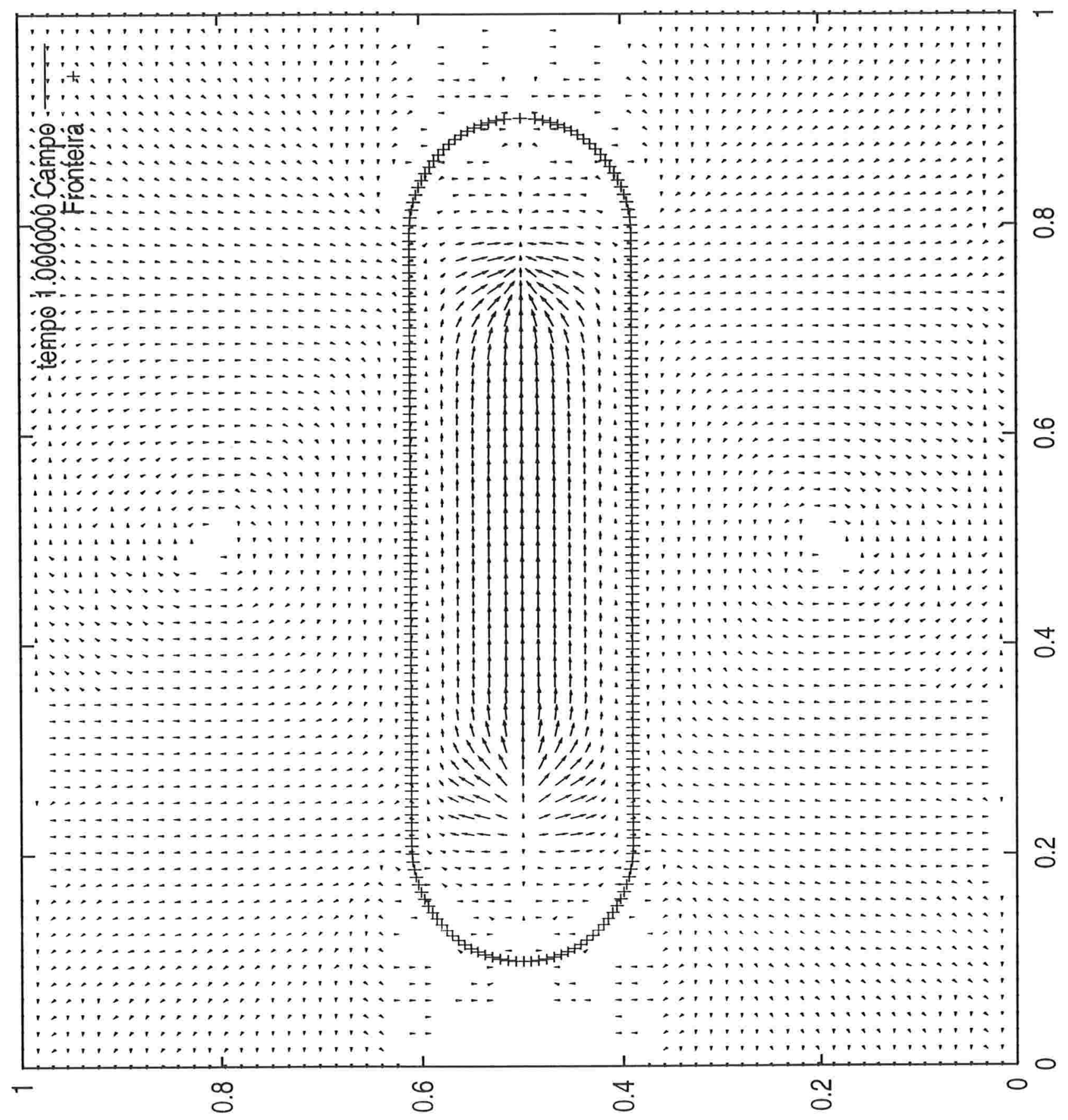

Figura 4.12: Quadro da simulação no tempo t=1.0; malha $64 \times 64$. 


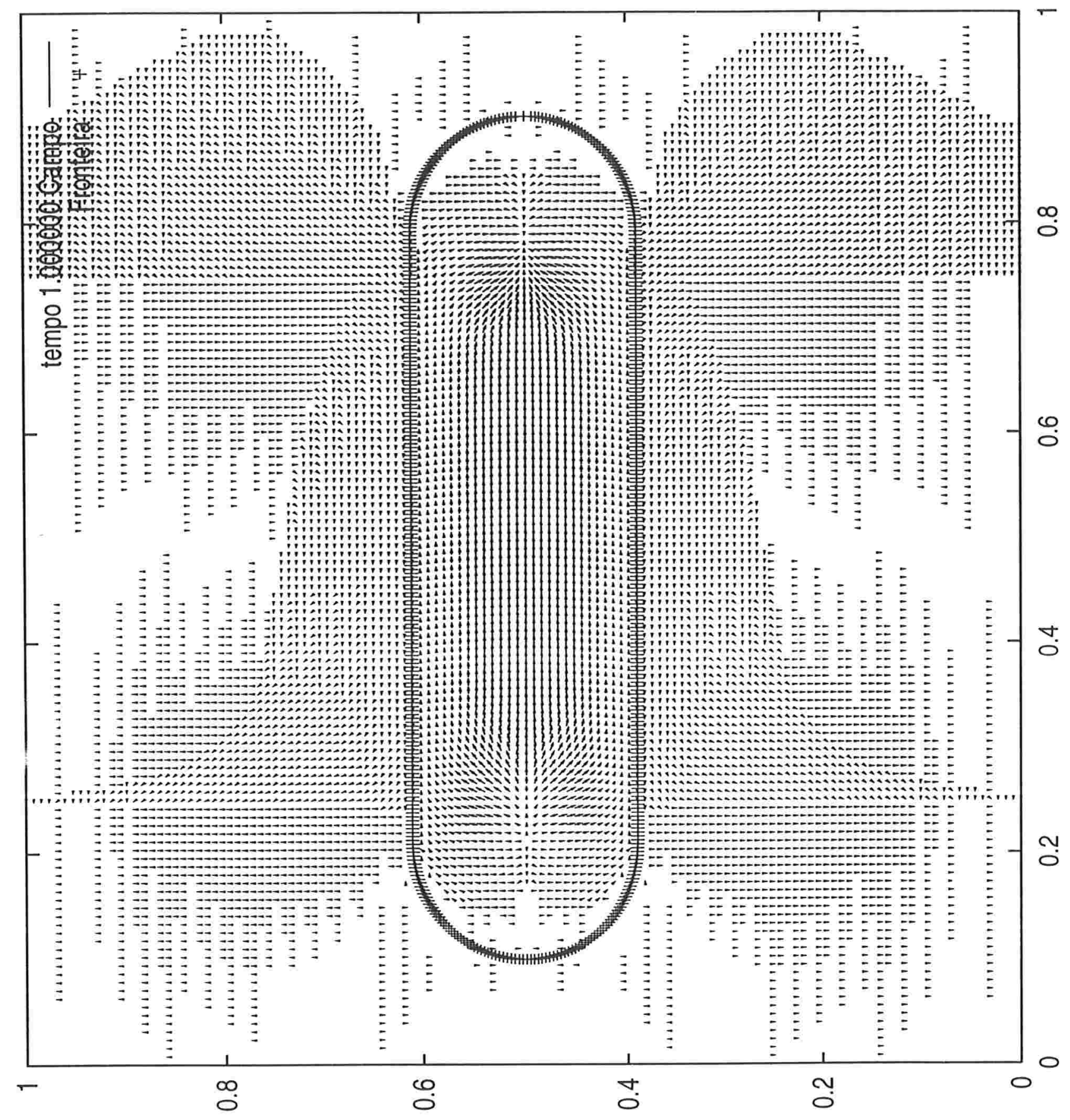

Figura 4.13: Quadro da simulação no tempo $t=1.0$; malha $128 \times 128$. 


\section{Conclusão}

O objetivo deste trabalho foi apresentar, implementar e testar adequadamente fonte e sumidouro, elementos necessários na modelagem matemática de entrada e saída de fluido em tubos flexíveis.

Foram elaborados testes que verificam o comportamento tanto do método numérico que resolve as equações do modelo como dos modelos adotados para a vazão na fonte e no sumidouro. O primeiro teste foi realizado com a finalidade de examinar a resolução numérica das equações de Navier-Stokes, o segundo para testar o desempenho do método na presença de uma fonte e um sumidouro e, finalmente, apresentou-se a simulação numérica do escoamento laminar bidimensional de fluido entre placas paralelas flexíveis, usando-se uma fonte e um sumidouro para simular o escoamento de fluido ao longo das placas.

\section{Conseguiu-se:}

1. verificar numericamente a convergência do passo de projeção empregado para resolver as equações de Navier-Stokes;

2. verificar o comportamento dos modelos adotados para a fonte e sumidouro, isto é, a conservação de massa no domínio todo;

3. apresentar a simulação numérica do escoamento laminar entre placas paralelas (secção longitudinal de um tubo) fazendo uso de uma fonte e de um sumidouro localizados no interior de um canal;

4. verificar a convergência de primeira ordem do Método da Fronteira Imersa, contendo uma fonte e um sumidouro, o que concorda com evidências computacionais encontradas por outros pesquisadores [17], [10], [19] e [3].

Além disso, conclui-se do teste de simulação de escoamento laminar entre placas paralelas (e do segundo teste): o escoamento desenvolvido com os parâmetros adotados para o modelo de resistência linear da vazão na fonte e no sumidouro atinge o regime permante e vê-se a 
necessidade do aumento do tamanho da malha para melhorar a resolução e convergência. Este problema poderia ser enfrentado usando um refinamento adaptativo de malhas (refinando uma vizinhança próxima da fronteira imersa) como o desenvolvido por Roma [17].

O modelo adotado para simular o escoamento laminar entre placas paralelas tem a forma de um canal bidimensional, imerso em um fluido, fechado nos extremos por semicircunferências colocadas para não impedir a fuga de massa fornecida pela fonte. As paredes deste canal são consideradas "flexíveis". Para manter a característica física do problema, constantes elásticas grandes foram utilizadas (similar ao adotado por Lai [10]). Este problema modelo, tomado para testar o desempenho da inclusão de fonte e sumidouro, pode ser visto também como o escoamento no interior de um tubo bidimensional com paredes flexíveis, motivação desta pesquisa.

Como continuidade deste trabalho, pretende-se empregar esta metodologia para modelar e simular problemas tridimensionais envolvendo tubos flexíveis. Implementações mais sofisticadas serão investigadas tais como, por exemplo, refinamento da malha de integração [17], uso de operadores divergente mais eficientes [16], e condições nas quais os tubos sofrem deformações graves [18]. 
APÊNDICE A

\section{Aproximação do Delta de Dirac}

\section{A.1 Função impulso unitário}

A função impulso unitário, chamada também de função Delta de Dirac, é "definida" como tendo as seguintes propriedades:

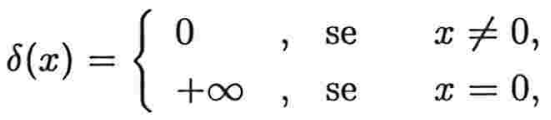

$$
\begin{aligned}
& \int_{-\infty}^{\infty} \delta(x) d x=1 \\
& \int_{-\infty}^{\infty} \delta(x) \phi(x) d x=\phi(0) \quad \phi \text { continua com suporte compacto }
\end{aligned}
$$

Aqui a palavra "função" não tem sentido formal, pois o Delta de Dirac não é uma função do ponto de vista clássico (exemplo, tem integral igual a 1 sendo nula em todo ponto excetuando-se o zero).

Dirac deu um sentido preciso a esta "função", introduzindo o que hoje chamamos de Núcleos de Dirac (ou regulizadores). No que segue, será necessário o conceito de função seccionalmente contínua. Uma função $f: \mathbf{R} \rightarrow \mathbf{R}$ será seccionalmente contínua se ela tiver apenas um número finito de discontinuidades (todas de primeira espécie) em qualquer intervalo limitado, ou seja, dados $a \leq b, \exists a_{i} i=1, \ldots, m / a \leq a_{1}<a_{2}<\ldots<a_{m} \leq b$ tais que $f$ é contínua em cada intervalo aberto $\left(a_{j}, a_{j+1}\right), j=1, \ldots, m-1$, e existem os limites

$$
f\left(a_{j}^{+}\right)=\lim _{x \rightarrow a_{j}^{+}} f(x) \quad \text { e } \quad f\left(a_{j}^{-}\right)=\lim _{x \rightarrow a_{j}^{-}} f(x)
$$




\section{A.2 Núcleos de Dirac}

\section{Definição}

Uma sucessão de funções $k_{n}: \mathbf{R} \rightarrow \mathbf{R}$ seccionalmente contínuas e satisfazendo às condições:

(D1) $\quad k_{n}(x) \geq 0, \quad \forall x \in \mathrm{R}$;

(D2) $\quad \int_{-\infty}^{\infty} k_{n}(x) d x=1$;

(D3) dados $\epsilon>0$ e $\eta>0$, existe um $n_{0}$ tal que, para $n \geq n_{0}, \int_{|x|>\eta} k_{n}(x) d x<\epsilon$

é chamada uma sucessão de Núcleos de Dirac.

\section{Definição}

O delta de Dirac é o limite de uma sucessão de núcleos de Dirac, isto é,

$$
\delta(x):=\lim _{n \rightarrow \infty} k_{n}(x), \quad x \in \mathrm{R},
$$

e além disso

$$
\int_{D \subset \mathrm{R}} \delta(x) \phi(x) d x:=\lim _{n \rightarrow \infty} \int_{D \subset \mathrm{R}} k_{n}(x) \phi(x) d x .
$$

Com base nestas definições, as propriedades (A.1)-(A.3) são verificadas, assim como

$$
\int_{D \subset \mathrm{R}} \delta(x) d x= \begin{cases}1, & 0 \in D, \\ 0, & 0 \notin D,\end{cases}
$$

\section{Observação}

Uma translação do delta de Dirac ao ponto $a$ é definida como

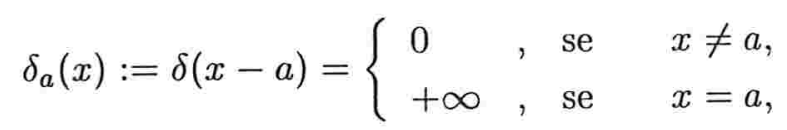

e que verifica as propriedades:

$$
\begin{aligned}
& \int_{D \subset \mathrm{R}} \delta(x-a) d x= \begin{cases}1, & a \in D, \\
0, & a \notin D,\end{cases} \\
& \int_{D \subset \mathrm{R}} \delta(x-a) \phi(x) d x=\phi(a),
\end{aligned}
$$

\section{Pergunta}

Como criar uma sucessão de núcleos de Dirac? Isto pode ser feito da seguinte forma: seja $k: \mathbf{R} \rightarrow \mathbf{R}$ uma função seccionalmente contínua, não negativa e tal que $0<\int_{-\infty}^{\infty} k(x) d x<\infty$. Se $\alpha=\int_{-\infty}^{\infty} k(x) d x$ então as funções $k_{n}(x)=\frac{n}{\alpha} k(n x), n \geq 1$, formam uma sucessão de núcleos de Dirac (a prova pode ser encontrada no livro de Djairo [6]). 
Em particular, $0<\int_{-\infty}^{\infty} k(x) d x<\infty$ se $k(x)$ for nula fora de um intervalo limitado.

A seguir, descreve-se um conjunto de aproximações para o delta de Dirac, as quais foram empregadas no Método da Fronteira Imersa, numa ou noutra oportunidade a forma de um elemento da sucessão de núcleos de Dirac é

$$
k_{n}(x)=d_{h}(x)=\frac{1}{h} \phi\left(\frac{x}{h}\right) . \quad h=\frac{1}{n} .
$$

A função $\phi(r)$ é contínua, não negativa, com suporte compacto e $\int_{-\infty}^{\infty} \phi(r) d r=1$. A primeira aproximação empregada por Peskin [13], for $\phi(r)=\phi^{(1)}(r)$, com suporte [-2,2], onde

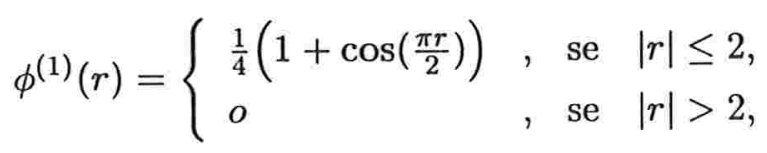

a qual satisfaz as seguintes propriedades:

1. $\phi$ é continua;

2. $\phi(r)=0, \quad \forall|r| \geq 2$;

3. $\sum_{i, p a r} \phi(r-i)=\sum_{i, \text { impar }} \phi(r-i)=1 / 2, \quad \forall r$

4. $\sum_{i}[\phi(r-i)]^{2}=c, \quad \forall r, \quad$ com $\quad c=3 / 8$.

As propriedades descritas acima têm por objetivos: primeiro, aproximar a função delta e, segundo, garantir a conservação de propriedades de interação entre a fibra e o fluido (descritas em [13]).

Entretanto, tal escolha não é a única. Peskin e McQueen no ano 1995 determinaram uma forma alternativa, $\phi(r)=\phi^{(2)}(r)$, que satisfaz a propriedade adicional:

$$
\text { 5. } \sum_{i}(r-i) \phi(r-i)=0, \quad \forall r
$$

Estas cinco propriedades determinam de forma única a função

$$
\phi^{(2)}(r)=\left\{\begin{array}{lll}
\frac{1}{8}\left(3-2|r|+\sqrt{1+4|r|-4 r^{2}}\right) & , \text { se }|r| \leq 1, \\
\frac{1}{8}\left(5-2|r|-\sqrt{-7+12|r|-4 r^{2}}\right) & , \text { se } 1 \leq|r| \leq 2, \\
0 & , \text { se }|r|>2 .
\end{array}\right.
$$

Outra forma da $\phi(r)=\phi^{(3)}(r)$, foi derivada por Roma [17], a qual satisfaz um conjunto similar de propriedades, tendo seu soporte reduzido a 1.5. A terceira propriedade reduz-se a $\sum_{i} \phi(r-i)=1 \mathrm{e}$, na quarta $c=1 / 2$.

$$
\phi^{(3)}(r)=\left\{\begin{array}{lll}
\frac{1}{6}\left(5-3|r|-\sqrt{-3(1-|r|)^{2}+1}\right) & , \text { se } 0.5 \leq|r| \leq 1.5 \\
\frac{1}{3}\left(1+\sqrt{-3 r^{2}+1}\right) & , \text { se }|r| \leq 0.5 \\
0 & , \text { se }|r|>0.5
\end{array}\right.
$$


Esta aproximação para a função delta é aplicável para o cálculo numa malha do tipo markerand-cell (MAC), onde o problema de desacoplamento na equação de Poisson para a pressão não aparece [17]. Outras expressões foram dadas por Beyer e Leveque [3], que são:

$$
\begin{aligned}
& \phi^{(4)}(r)= \begin{cases}1-|r| & , \text { se }|r| \leq 1, \\
0 & , \quad|r|>1,\end{cases} \\
& \phi^{(5)}(r)= \begin{cases}\left(1-r^{2}\right) & , \text { se }|r| \leq 1, \\
\left(2-3|r|+r^{2}\right) & , \text { se } 1 \leq|r| \leq 2, \\
0 & , \quad|r|>2 .\end{cases}
\end{aligned}
$$

Na Figura A.1, mostra-se os gráficos das funções $\phi(r)=\phi^{(k)}(r), k=1, \ldots, 5$, no domínio [-2, 2].

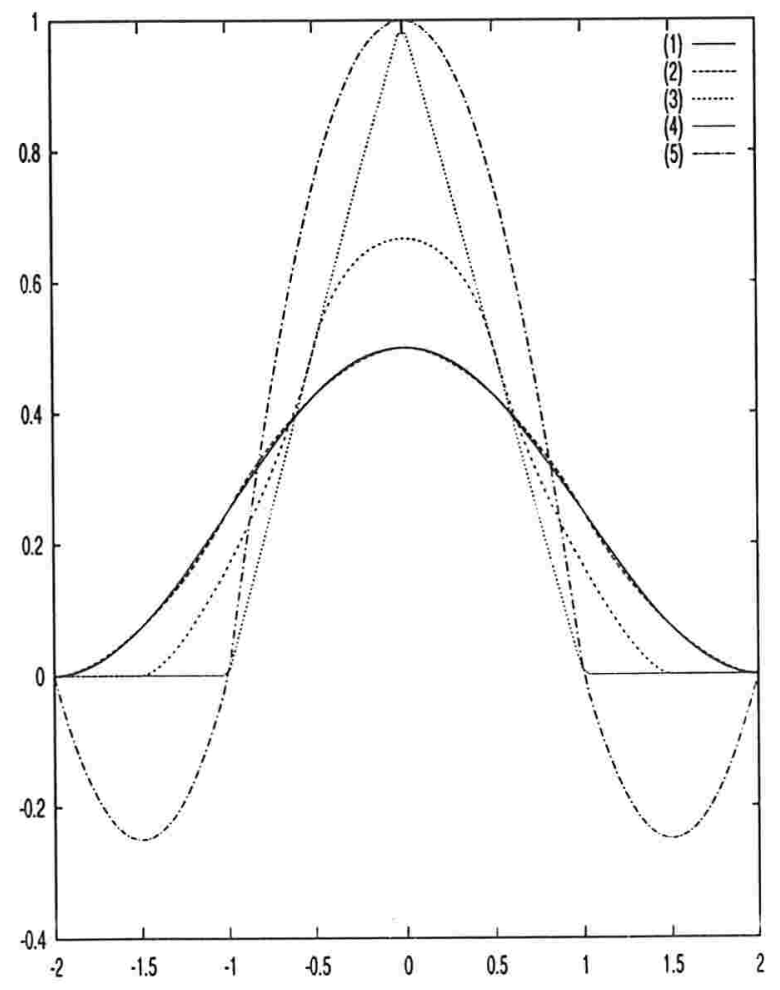

Figura A.1: Gráfico das funções $\phi(r):(1) \phi^{(1)} ;(2) \phi^{(2)} ;(3) \phi^{(1)} ;(4) \phi^{(4)} ;(5) \phi^{(5)}$. 


\section{Decomposição de um campo vetorial (caso periódico)}

O objetivo deste apêndice é apresentar a prova do Teorema de Decomposição de Hodge-Helmholtz em sua versão periódica (Teorema 1, Seção 2.2).

\section{B.1 Preliminares: definições e propriedades}

1. Dados os campos escalares

$$
r, s \in\left\{h:\left.\Omega \subset \mathrm{R}^{2} \rightarrow \mathrm{R}\left|\int_{\Omega}\right| h(\mathrm{x})\right|^{2} d \mathrm{x}<\infty\right\},
$$

e vetoriais

$$
\mathbf{u}, \mathbf{v} \in L^{2}(\Omega)=\left\{\mathbf{h}=\left(h_{1}, h_{2}\right): \Omega \subset \mathrm{R}^{2} \rightarrow \mathrm{R}^{2} \mid \int_{\Omega}\left(\left(h_{1}(\mathrm{x})\right)^{2}+\left(h_{2}(\mathrm{x})\right)^{2}\right)^{2} d \mathbf{x}<\infty\right\}
$$

os produtos internos para funções escalares e vetoriais podem ser definidas respectivamente como

$$
\begin{aligned}
r \cdot s & =\int_{\Omega} r(\mathbf{x}) s(\mathbf{x}) d \mathbf{x} \\
\mathbf{u} \cdot \mathbf{v} & =\sum_{i=1}^{2} u_{i} \cdot v_{i}
\end{aligned}
$$

onde $\mathbf{u}=\left(u_{1}, u_{2}\right)$ e $\mathbf{v}=\left(v_{1}, v_{2}\right)$.

2. Do livro de Kaplan [9], temos

Teorema 2 (Teorema da Green) Seja $D$ um dominio do plano xy e seja $C$ uma curva simples, fechada, lisa por partes, contida em $D$ e cujo interior também está em D. Sejam 
$P(x, y)$ e $Q(x, y)$ duas funções definidas e contínuas em $D$, possuindo derivadas primeiras contínuas. Nessas condições, vale

$$
\oint_{C} P d x+Q d y=\int_{R} \int\left(\frac{\partial Q}{\partial x}-\frac{\partial P}{\partial y}\right) d x d y
$$

onde $R$ é a região fechada limitada por $C$.

Seja o campo vetorial $\mathbf{u}(x, y)=(u(x, y), v(x, y))$ com $u$ e $v$ funções definidas e contínuas em $D$, possuindo derivadas primeiras contínuas. Consideremos $P=-v$ e $Q=u$, usando-se o teorema de Green

$$
\oint_{C}-v d x+u d y=\int_{R} \int\left(\frac{\partial u}{\partial x}+\frac{\partial v}{\partial y}\right) d x d y
$$

O integrando do lado direito de (B.2) é o divergente do campo vetorial $\mathbf{u}, \nabla \cdot \mathbf{u}$, enquanto a integral do lado esquerdo pode-se expressar, usando-se o elemento de comprimento $d s$, como

$$
\oint_{C} \mathbf{u} \cdot \mathbf{n} d s
$$

onde $\mathbf{n}$ é o vetor normal unitário exterior ao longo da curva $C$. Logo, a equação B.2 pode-se escrever como

$$
\oint_{C} \mathbf{u} \cdot \mathbf{n} d s=\int_{R} \int \nabla \cdot \mathbf{u} d x d y .
$$

Esse resultado é a versão em duas dimensões do Teorema da Divergência ou de Gauss, a qual se enuncia a seguir

3. Teorema 3 (Teorema da Divergência) Sejam V um sólido no espaço tridimensional limitado por uma superfície orientável $\partial \mathbf{V}$ e $\mathbf{n}$ a normal exterior de $\partial \mathbf{V}$. Se $\mathbf{F}$ é um campo vetorial continuamente diferenciável em $\Omega$, tem-se

$$
\int_{\mathrm{V}} \nabla \cdot \mathbf{F} d \mathbf{x}=\int_{\partial \mathrm{V}} \mathbf{F} \cdot \mathbf{n} d \sigma(\mathbf{x})
$$

\section{Primeira identidade de Green}

Sejam dois campos escalares $r$ e s contínuos no domínio $\Omega$. com segundas derivadas contínuas no dominio, então

$$
\int_{\Omega}(r \Delta s+\nabla r \cdot \nabla s) d \mathbf{x}=\int_{\partial \Omega} r \frac{\partial s}{\partial \mathbf{n}} d \sigma(\mathrm{x})
$$

Em particular, se $r=1$, obtém-se:

$$
\int_{\Omega} \Delta s d \mathbf{x}=\int_{\partial \Omega} \frac{\partial s}{\partial \mathbf{n}} d \sigma(\mathbf{x}) .
$$

Uma aplicação deste resultado particular é a condição de compatibilidade para a equação de Poisson com condições de fronteira de Dirichlet, a qual tem a seguinte forma:

$$
\left\{\begin{aligned}
\Delta p(\mathbf{x})=f(\mathbf{x}), & \mathbf{x} \in \Omega, \\
p(\mathbf{x})=g(\mathbf{x}), & \mathbf{x} \in \partial \Omega .
\end{aligned}\right.
$$


Usando-se o caso particular da primeira identidade de Green para $s=p$ (solução da equação de Poisson B.5), obtém-se:

$$
\int_{\Omega} f(\mathbf{x}) d \mathbf{x}=\int_{\partial \Omega} \nabla g(\mathbf{x}) \cdot \mathbf{n}(\mathbf{x}) d \sigma(\mathbf{x})
$$

onde $\mathbf{n}$ é o vetor normal unitário exterior à fronteira de $\Omega, \partial \Omega$. Está condição necessária devem ser satisfeitas pelas funções $f$ e $g$ (condição de compatibilidade para $f$ e $g$ ).

\section{B.2 Prova do Teorema 1 (Seção 2.2.2)}

\section{Teorema}

Qualquer campo vetorial periódico $\mathbf{w}$ definido em um retângulo $\Omega$ pode ser unicamente decomposto na forma

$$
\mathbf{w}=\mathbf{u}^{d}+\nabla \Phi
$$

onde $\mathbf{u}^{d}$ é um campo vetorial periódico tal que $\nabla \cdot \mathbf{u}^{d}=0$ e $\Phi$ uma função escalar periódica.

\section{Prova}

- Os campos $\mathbf{u}^{d}$ e $\nabla \Phi$ são ortogonais.

De fato, usando-se a identidade

$$
\nabla \cdot(f \mathbf{F})=(\nabla \cdot \mathbf{F}) f+\mathbf{F} \cdot \nabla f
$$

para $f=\phi$ e $\mathbf{F}=\mathbf{u}^{d}$, e sendo $\nabla \cdot \mathbf{u}^{d}=0$, resulta que

$$
\mathbf{u} \cdot \nabla \Phi=\nabla \cdot\left(\Phi \mathbf{u}^{d}\right)-\left(\nabla \cdot \mathbf{u}^{d}\right) \Phi=\nabla \cdot\left(\Phi \mathbf{u}^{d}\right) .
$$

Devido ao teorema da divergência e condição de fronteira periódica no domínio retangular, obtém-se

$$
\int_{\Omega} \mathbf{u} \cdot \nabla \Phi d \mathbf{x}=\int_{\Omega} \nabla \cdot\left(\Phi \mathbf{u}^{d}\right) d \mathbf{x}=\int_{\Omega} \Phi \mathbf{u}^{d} \cdot n(\mathbf{x}) d \sigma(\mathbf{x})=0
$$

- Unicidade de soluções.

Suponha que: $\mathbf{w}=\mathbf{u}_{1}^{d}+\nabla \Phi_{1}=\mathbf{u}_{2}^{d}+\nabla \Phi_{2}$, então

$$
\mathbf{u}_{1}^{d}-\mathbf{u}_{2}^{d}+\nabla\left(\Phi_{1}-\Phi_{2}\right)=0 \text {. }
$$

Realizando-se o produto interno entre $\left(\mathbf{u}_{1}^{d}-\mathbf{u}_{2}^{d}\right)$ e a expressão anterior, encontra-se

$0=\int_{\Omega}\left(\left\|\mathbf{u}_{1}^{d}-\mathbf{u}_{2}^{d}\right\|^{2}+\left(\mathbf{u}_{1}^{d}-\mathbf{u}_{2}^{d}\right) \cdot \nabla\left(\Phi_{1}-\Phi_{2}\right)\right) d \mathbf{x}=\int_{\Omega}\left\|\mathbf{u}_{1}^{d}-\mathbf{u}_{2}^{d}\right\|^{2} d \mathbf{x}+\int_{\Omega}\left(\mathbf{u}_{1}^{d}-\mathbf{u}_{2}^{d}\right) \cdot \nabla\left(\Phi_{1}-\Phi_{2}\right) d \mathbf{x}$ pela relação de ortogonalidade, o segundo membro do lado direito é nulo, logo

$$
0=\int_{\Omega}\left\|\mathbf{u}_{1}^{d}-\mathbf{u}_{2}^{d}\right\|^{2} d \mathbf{x} \rightarrow \mathbf{u}_{1}^{d}=\mathbf{u}_{2}^{d}
$$


e assim, $\nabla \Phi_{1}=\nabla \Phi_{2}$, que implica que $p_{1}=p_{2}+$ constante.

-Existência

Aplicando-se o operador divergente na expressão (B.7) e usando-se a condição para $\mathbf{u}^{d}, \nabla \cdot \mathbf{u}^{d}=0$, tem-se

$$
\Delta \Phi=\nabla \cdot \nabla \Phi=\nabla \cdot \mathbf{w}
$$

A equação de Poisson (B.8) (com $f=\nabla \cdot \mathbf{w}$, e $g$ função periódica na fronteira) satisfaz a condição de compatibilidade (B.6) (usando-se o teorema da divergência).

No livro de Weinberger [20] está provado a existência para equações de Poisson (B.5) definidas em um retângulo da forma $[0, \pi] \times[0, A]$. Primeiro, resolve a equação não homogênea com condição de fronteira nula e segundo, a equação homogênea com condição de fronteira não nula, associadas a tal problema. A demonstração está baseada na construção da função de Green do respectivo problema. Tal existência continua sendo válida em qualquer outro retângulo, por exemplo $\Omega$, para isso é necessário somente que se faça uma mudança de coordenadas. 


\section{Teorema do Transporte}

A mudança ao longo do tempo das propriedades físicas de um fluido que ocupa uma região $\Omega$ do plano ou espaço podem ser descritas de duas maneiras possíveis. A primeira, denominada Euleriana, descreve estas mudanças para cada ponto fixado no domínio físico. A segunda, denominada Lagrangeana, descreve estas mudanças ao longo das trajetórias seguidas por cada partícula do fluido, identificando-as por intermédio de um parâmetro escolhido convenientemente, denominado parâmetro lagrangiano ou coordenada material. Comumente, escolhe-se a posição inicial como coordenada material.

Considere uma porção de fluido que no instante inicial $t=t_{0}$, ocupa uma região do espaço $\Omega_{0} \subset \Omega$. Para descrever seu movimento, tome a função $\phi(\mathbf{a}, t)$, chamada função fluxo, tal que para cada a $\in \Omega_{0}$ (coordenada material), a curva $t \mapsto \phi(\mathbf{a}, t)$ descreve a trajetória da partícula que ocupara a posição a no instante $t=t_{0}$. Um domínio material $\Omega_{t}$ é um subdomínio de $\Omega$ que satisfaz

$$
\Omega_{t}=\phi_{t}\left(\Omega_{0}\right)
$$

Na Figura C.1 mostra-se este domínio.

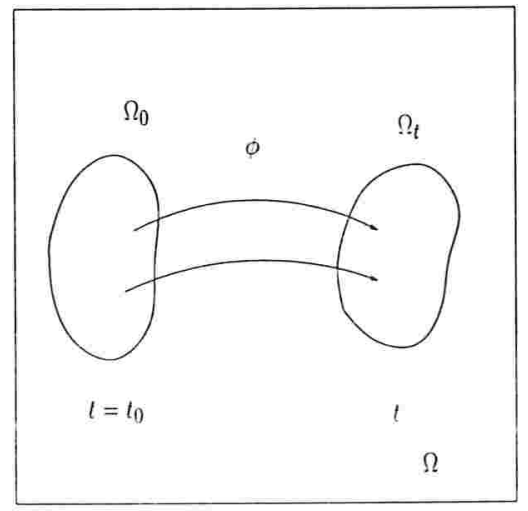

Figura C.1: Região do espaço ocupado pelo fluido no instante t, $\Omega_{t}$. 
É necessário que a função fluxo, $\phi$, possua propriedades de diferenciabilidade e invertibilidade. Mais precisamente, a função

$$
\begin{aligned}
\phi_{t}: \Omega_{0} & \longrightarrow \Omega_{t} \\
\mathrm{a} & \longmapsto \phi(\mathrm{a}, t)
\end{aligned}
$$

seja diferenciável e possua inversa diferenciável.

Seja $\mathbf{u}(\mathbf{x}, t)$ a velocidade do fluido da partícula que, no instante $t$, ocupa a posição $\mathbf{x}$. A seguinte relação segue-se imediatamente das definições

$$
\frac{\partial \phi}{\partial t}(\mathbf{a}, t)=\mathbf{u}(\phi(\mathbf{a}, t), t)
$$

Se o campo velocidade for conhecido, obtém-se $\phi(\mathbf{a}, t)$ resolvendo-se, para cada $\mathbf{a} \in \Omega_{0}$, a equação diferencial ordinária com condição inicial

$$
\begin{aligned}
\frac{\partial \phi}{\partial t}(\mathbf{a}, t) & =\mathbf{u}(\phi(\mathbf{a}, t), t) \\
\phi\left(\mathbf{a}, t_{0}\right) & =\mathbf{a} .
\end{aligned}
$$

\section{C.0.1 Derivada Material e teorema de transporte}

Dada uma função $f(\mathbf{x}, t), \mathbf{x} \in \Omega_{t}$, e a trajetória $\phi(\mathbf{a}, t)$ para algum $\mathbf{a}$, com $\mathbf{x}=\phi(\mathbf{a}, t)$ a função $f(\phi(\mathrm{a}, t), t)$ descreve o valor da função ao longo da trajetória. Definimos a derivada material de $f$ como:

$$
\frac{\mathrm{D} f}{\mathrm{D} t}(\mathbf{x}, t):=\frac{d f}{d t}(\phi(\mathbf{a}, t), t)=\frac{\partial f}{\partial t}(\mathbf{x}, t)+\nabla f(\mathbf{x}, t) \cdot u(\mathbf{x}, t)
$$

então a derivada material de $f, \frac{\mathbf{D}_{f}}{\mathbf{D}_{t}}$, fornece o valor no instante t da derivada de $f$ ao longo da trajetória da partícula que no instante $\mathrm{t}$, ocupa a posição $\mathrm{x} \in \Omega_{t}$. Nas aplicações, pode desempenhar o papel de $f$, por exemplo, a densidade da massa, a temperatura, ou mesmo a própria velocidade.

Teorema 4 (Teorema do transporte) Satisfeitas as hipóteses sobre a função fluxo $\phi$ mencionadas acima e sendo $\Omega_{t}$ uma região onde se pode aplicar o Teorema da Divergência, com $f \in \mathbf{C}^{1}\left(\bar{\Omega}_{t}\right)$, vale a seguinte identidade

$$
\frac{d}{d t}\left(\int_{\Omega_{t}} f(\mathbf{x}, t) d \mathbf{x}\right)=\int_{\Omega_{t}}\left(\frac{\mathbf{D} f}{\mathbf{D} t}+f \cdot \nabla u\right)(\mathbf{x}, t) d \mathbf{x} .
$$




\section{Cálculo da área de polígonos}

\section{D.1 Fórmula integral do cálculo da área}

Se $\mathbf{u}: \Omega \subset \mathbf{R}^{2} \rightarrow \mathbf{R}^{2}$ for uma função cumprindo as condições do Teorema da Divergência em duas dimensões (Apêndice B, relação (B.3)) então tem-se

$$
\int_{\Omega} \nabla \cdot \mathbf{u} d \mathbf{x}=\int_{\partial \Omega} \mathbf{u} \cdot \mathbf{n} d \sigma(\mathbf{x})
$$

onde $\mathbf{n}$ é o vetor normal unitário exterior à fronteira $\partial \Omega$ e $d \sigma(\mathbf{x})$ é o elemento de comprimento ao longo da fronteira. Se $\mathbf{u}(x, y)$ for a função identidade, isto é, $\mathbf{u}(x, y)=(x, y)$, então, por aplicação do Teorema da Divergência, obtém-se

$$
\int_{\Omega} \nabla \cdot \mathbf{u} d \mathbf{x}=2 \int_{\Omega} d \mathbf{x}=\int_{\partial \Omega} \mathbf{u} \cdot \mathbf{n} d \sigma(\mathbf{x})
$$

Como Área $(\Omega)=\int_{\Omega} d \mathbf{x}$, tem-se, da igualdade acima, que

$$
\text { Área }(\Omega)=\frac{1}{2} \int_{\partial \Omega} \mathbf{u} \cdot \mathbf{n} d \sigma(\mathbf{x})
$$

No caso da fronteira de $\Omega$ ter a forma de um polígono formado por $n$ vértices $P_{1}, P_{2}, \ldots, P_{n}$, conforme ilustrado na Figura D.1, a expressão dada na fórmula (D.1), pode ser escrita como

$$
\operatorname{Área}(\Omega)=\frac{1}{2}\left(\sum_{i=1}^{n} \int_{L_{i}} \mathbf{u} \cdot \mathbf{n} d \sigma(\mathrm{x})\right),
$$

onde $L_{i}$ é a i-ésima aresta $i=1,2, \ldots, n$, dada por

$$
L_{i}=\left\{P(s) \mid \quad P(s)=P_{i}+\left(P_{i+1}-P_{i}\right) s, \quad s \in[0,1]\right\}, \quad P_{n+1}=P_{1}
$$

De Álgebra Linear, sabe-se que dado dois vetores A e B com origens em $\mathrm{O}$ a área do triângulo determinado por eles é dada por

$$
\operatorname{Area}(\mathbf{A}, \mathbf{B})=( \pm) \frac{\mathbf{A} \cdot \mathbf{B}^{\perp}}{2}
$$




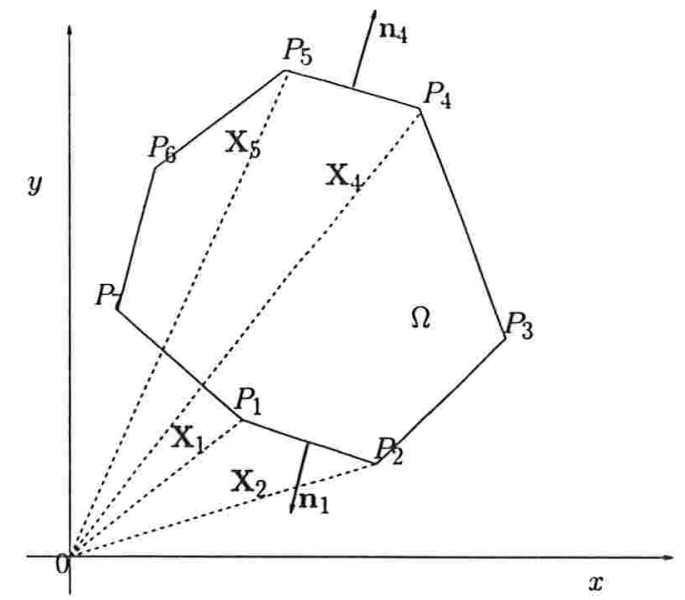

$\mathrm{O}(=$ Origem $)$

Figura D.1: Fronteira de $\Omega(\partial \Omega)$ com forma de polígono.

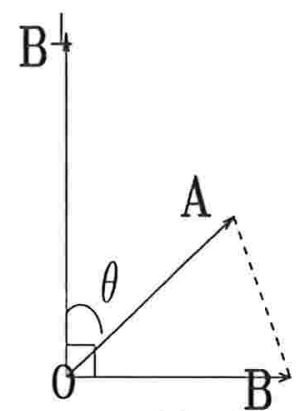

(a)

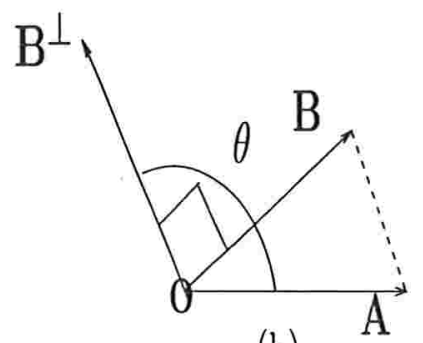

(b)

Figura D.2: Ángulos possíveis formados por A e $\mathrm{B}^{\perp}$ : (a) agudo e (b) obtuso.

onde a escolha do sinal "+" e "-" depende do posicionamento dos vetores.

A Figura (D.2) mostra as possibilidades se o ângulo formado entre eles for agudo, Figura (D.2)-(a), o sinal é positivo. Neste contexto, cada uma das integrais que aparecem em (D.2) é dada por

$$
\int_{L_{i}} \mathbf{u} \cdot \mathbf{n} d \sigma(\mathbf{x})=( \pm) 2 \text { Área }\left(\triangle 0 P_{i} P_{i+1}\right), \quad i=1, \ldots, n,
$$

onde a escolha do sinal depende do vetor normal: se esse vetor aponta para fora da região triangular $0 P_{i} P_{i+1}$ então o sinal escolhido é o positivo, e negativo no caso contrário. Esta relação é facilmente verificada usando-se a expressão para u no caminho $L_{i}$ e a relação anterior para a área formada por dois vetores dada em (D.3). Finalmente, a fórmula para a área de uma 
região poligonal está dada por

$$
A(\Omega)=\left(\sum_{i=1}^{n}( \pm) \text { Área }\left(\triangle 0 P_{i} P_{i+1}\right)\right)
$$

onde os sinais "+" e "-" seguem a consideração feita no item anterior. 


\section{Referências Bibliográficas}

[1] Kayne M. Arthurs, Leon C. Moore, Charles S. Peskin, E. Bruce Pitman e H. E. Layton, Modeling arteriolar flow and mass transport using the immersed boundary method, $J$. Comput. Phys. 147 (1998), no. 2, 402-440.

[2] R P. Beyer. A computational model of cochlea using the immersed boundary method, Journal of Computational Physics, 98 (1992), 145-162.

[3] R P. Beyer e R J. LeVeque, Analysis of a one-dimensional model for the immersed boundary method, SIAM Journal on Numerical Analysis, 29 (1992), no. 2, 332-364.

[4] Alexandre J. Chorin, Numerical solution of the navier-stokes equations, Mathematics of Computation, 22 (1968), no. 104, 745-762.

[5] Alexandre J. Chorin e J E. Marsden, A mathematical introduction to fluid mechanics, second ed., Springer-Verlag, 1990.

[6] G F. Djairo, Análise de fourier e equações diferenciais parciais, 3 ed., Projeto Euclides.

[7] Lisa J. Fauci e Charles S. Peskin, A computational model of aquatic animal locomotion, $J$. Comput. Phys. 77 (1988), no. 1, 85-108.

[8] Aaron L. Fogelson, A mathematical model and numerical method for studying platelet adhesion and aggregation during blood clotting, J. Comput. Phys. 56 (1984), no. 1, 111134.

[9] Wilfred. Kaplan, Cálculo avançado, vol. 1, Edgar Blücher Ltda., 1972.

[10] Ming-chih. Lai, Simulation of the flow past an array of circular cylinders as a test of the immersed boundary method, Ph.D. thesis, New York University, 1998. 
[11] M S. Oshiro, S A. Hayashida, M J S. Maizatto, E F. Marques, N A G. Stolf, A D. Jetene e A A. Leirner, Design, manufacturing, and testing of paracorporeal pulsatile ventricular assit device: Sąo paulo heart institute v a d, Artificial Organs 19 (1995), no. 3, 274-279.

[12] Charles S. Peskin, Flow patterns around heart valves: A numerical method, Journal of Computational Physics, 10 (1972), 252-271.

[13] - Numerical analysis of blood flow in the heart, J. Computational Phys. 25 (1977), no. $3,220-252$.

[14] _ Fiber architecture of the left ventricular wall: an asymptotic analysis, Comm. Pure Appl. Math. 42 (1989), no. 1, 79-113.

[15] Charles S. Peskin e David M. McQueen, A three-dimensional computational method for blood flow in the heart. I. Immersed elastic fibers in a viscous incompressible fluid, $J$. Comput. Phys. 81 (1989), no. 2, 372-405.

[16] Charles S. Peskin e Beth Feller. Printz, Improved volume conservation in the computation of flows with immersed elastic boundaries, J. Comput. Phys. 105 (1993), no. 1, 33-46.

[17] Alexandre M. Roma, A multilevel self adaptive version of the immersed boundary method, Ph.D. thesis, New York University, 1994.

[18] Madeleine E. Rosar, A three-dimensional computer model for fluid flow through a collapsible tube, Ph.D. thesis, Courant Institute of Mathematical Sciences-New York University, June 1994.

[19] John Michael. Stockie, Analysis and computation of immersed boundaries, with application to pulp fibres, Ph.D. thesis, University of British Columbia, 1997.

[20] H F. Weinberger, Ecuaciones diferenciales en derivadas parciales., Editorial Reverté, S. A., 1970.

[21] R.M.S. Yoshikawa e A.M. Roma, Estudo da interação fluido-fronteira através do método da fronteira imersa, Simpósio de Iniciação Cientifíca da Universidade de São Paulo, 6, vol. 2, 1998, p. 235. 